\title{
Interpenetrating Polymer Networks Templated on Bicontinuous Microemulsions Containing Silicone Oil, Methacrylic Acid and Hydroxyethyl Methacrylate
}

by

Victor Stanislaus Castellino

A thesis submitted in conformity with the requirements

for the degree of Doctor of Philosophy

Chemical Engineering and Applied Chemistry

University of Toronto

(C) Copyright by Victor Castellino 2011 


\title{
Interpenetrating Polymer Networks Templated on Bicontinuous Microemulsions Containing Silicone Oil, Methacrylic Acid and Hydroxyethyl Methacrylate
}

\author{
VICTOR CASTELLINO \\ Doctor of Philosophy \\ Chemical Engineering and Applied Chemistry \\ University of Toronto
}

2011

\begin{abstract}
Interest in microemulsions as potential platforms for polymerization stems from the wide range of phase behaviour dependant morphologies and domain sizes that can be generated in a low viscosity environment. By introducing polymerizable components into the oil and aqueous phases of a microemulsion, we may essentially create a low viscosity, low interfacial tension, bicontinuous template with nanostructured morphologies and narrow domain size distributions analogous to those generated through conventional interpenetrating polymer network (IPN) synthesis and spinodal decomposition. The main objective of this dissertation is to test the application of bicontinuous microemulsion templates to the formulation and polymerization of a silicone-hydrogel IPN. In addition, the project expands on the classical definition of IPNs to a scale of entanglement at the level of groups of polymer chains, as opposed to molecular or chain-level entanglement.
\end{abstract}

This study is divided into two main parts. In the first part, silicone microemulsions were developed and characterized according to the Hydrophilic-Lipophilic Difference (HLD) framework. The hydrophobicity of silicone oils, the characteristic curvature of silicone 
surfactants and the co-surfactant contribution of methacrylic acid (MAA) and hydroxyethyl methacrylate (HEMA) were quantified. These findings led to the successful formulation of bicontinuous microemulsions ( $\mu \mathrm{Es})$ containing silicone oil, silicone alkyl polyether and reactive monomers in aqueous solution. Ternary phase diagrams of these systems revealed the potential for silicone-containing polymer composites with bicontinuous morphologies.

In the second part of this study, the formulation and simultaneous polymerization of polydimethylsiloxane-poly(methacrylic acid - hydroxyethyl methacrylate), (PDMS-P(MAAHEMA) IPNs from bicontinuous microemulsions was demonstrated. Laser scanning confocal microscopy (LSCM) on swollen polymers highlights aqueous pathways, and indicates the formation of bicontinuous morphologies with domain sizes at equilibrium swelling ranging from $\sim 100 \mathrm{~nm}$ to $1 \mu \mathrm{m}$. Incorporating polymerizable surfactants into the microemulsion aided in stabilizing the initial microemulsion structure during polymerization. The process developed demonstrates a simple, single-step polymerization approach to forming IPNs from low viscosity microemulsion templates, and could potentially be extended to a variety of hydrophilic and hydrophobic monomers. 


\section{Acknowledgments}

I would first like to express my gratitude to my supervisors, Professors Yu-Ling Cheng and

Edgar Acosta, who have stuck with me through all the twists and turns in my studies. Thank you also to my committee members past and present: Professors Masahiro Kawaji, Tim Bender and Yu Sun. Beyond feedback and suggestions for improving my work, your eagerness to see me progress is much appreciated.

Thank you to Dr. John Oreopoulos and Professor Christopher Yip for finding the time to walk me through the LSCM work that that critical to this project's success.

Thank you to Professor Joseph Paradi for reminding me of the world outside the lab, and for showing me that engineering is about more than mass balances.

Thank you to my family for your love and support through 10 years of school.

And finally, thank you to my wife, Adriane Castellino, for believing in me through the highs and lows of my $\mathrm{PhD}$ work, and for agreeing that this was, after all, a good idea. 


\section{Table of Contents}

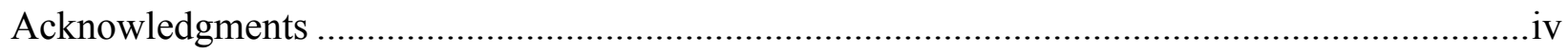

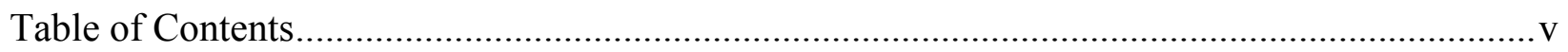

List of Abbreviations and Symbols ...................................................................................

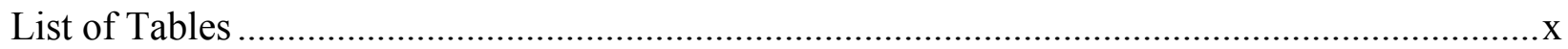

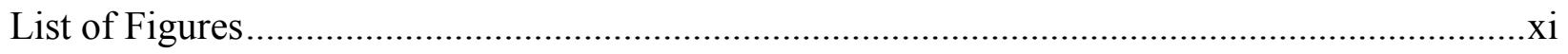

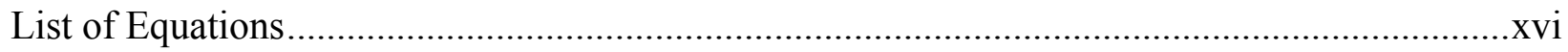

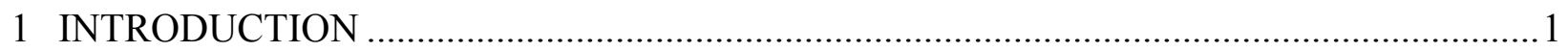

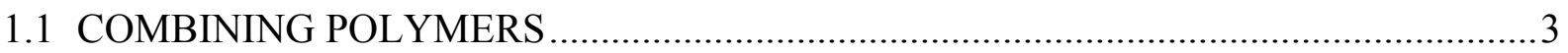

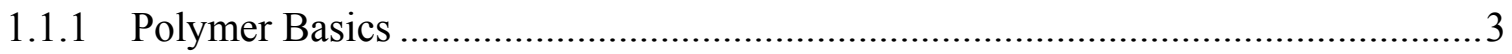

1.1.2 Thermodynamics and Phase Behaviour ............................................................

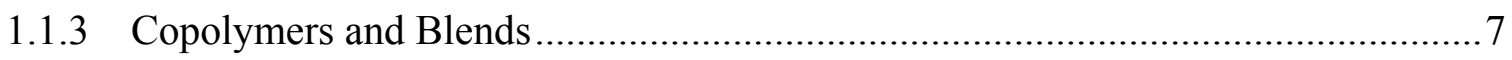

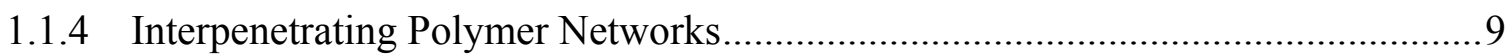

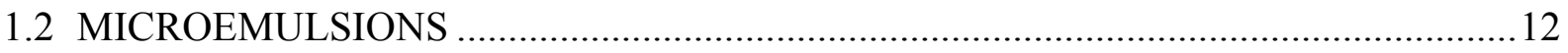

1.2.1 Thermodynamics of Surfactant Systems ……..................................................... 14

1.2.2 Microemulsion Structure and Behaviour.............................................................15

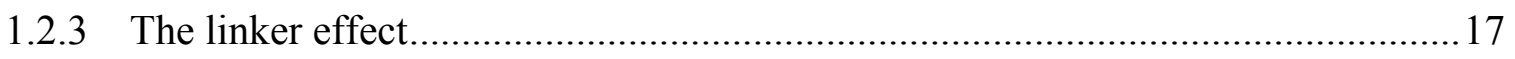

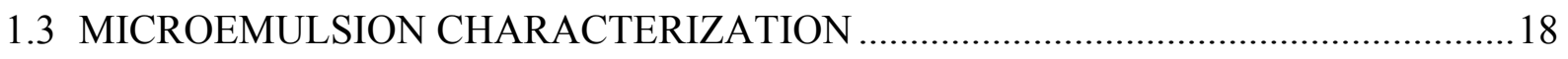

1.3.1 Persistence Length ........................................................................................19

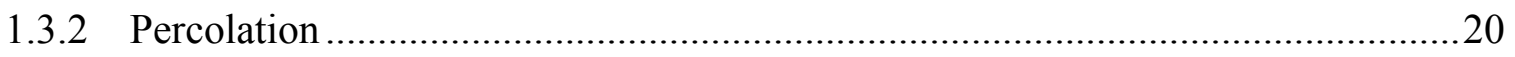

1.3.3 The Winsor Ratio.......................................................................................21

1.3.4 Hydrophilic - Lipophilic balance (HLB) and Phase Inversion Temperature........23

1.3.5 Hydrophilic-Lipophilic Difference (HLD) ……………..................................2.

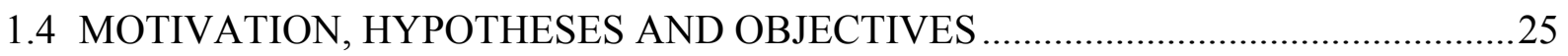

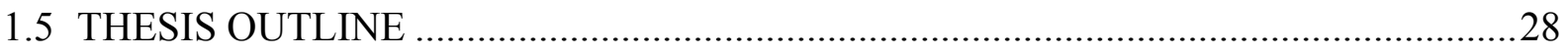


1.6 REFERENCES

2 THE HYDROPHOBICITY OF SILICONE-BASED OILS AND SURFACTANTS AND THEIR USE IN REACTIVE MICROEMULSIONS

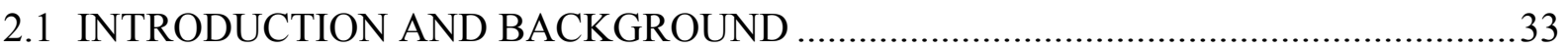

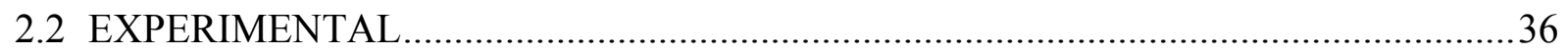

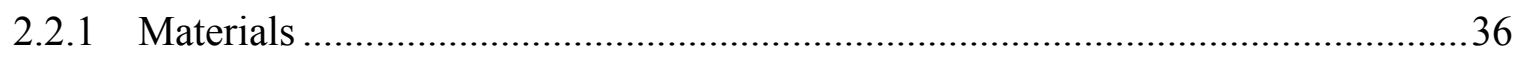

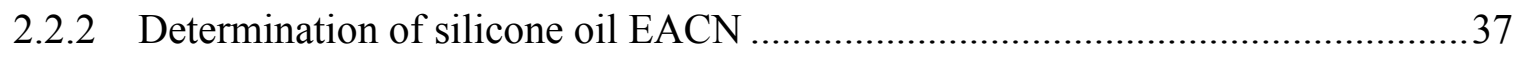

2.2.3 Determining the characteristic curvature of silicone surfactants............................

2.2.4 Aqueous solution containing acrylic monomers........................................40

2.2.5 Microemulsion preparation and phase behaviour scans .................................. 41

2.2.6 Microemulsion characterization: conductance, microscopy and viscosity...........42

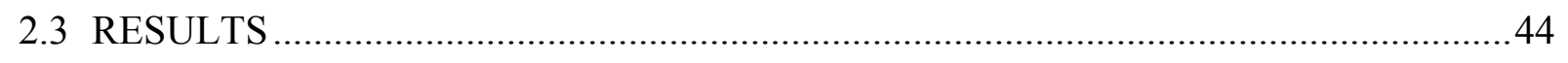

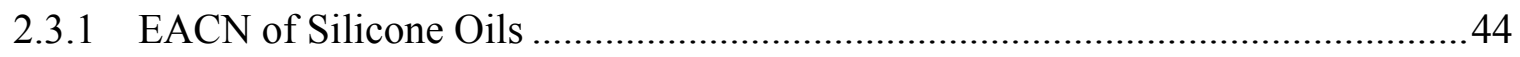

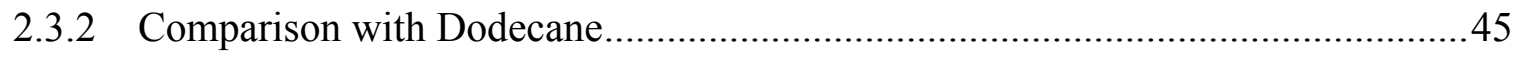

2.3.3 Characteristic Curvature of Silicone Alkyl Polyether Surfactants ......................46

2.3.4 HLD Equation for Silicone-based Microemulsions and the Effect of

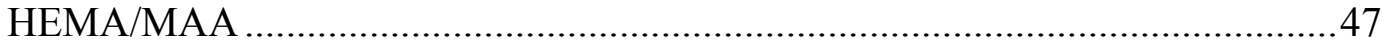

2.3.5 Effect of Temperature and $\mathrm{pH}$ on Phase Behaviour .......................................51

2.3.6 Characterization of the homogeneous region by electrical conductance and

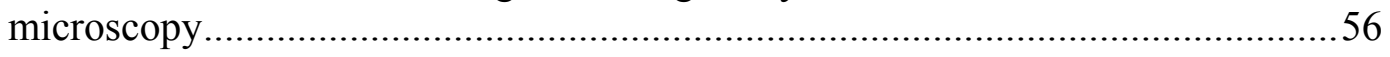

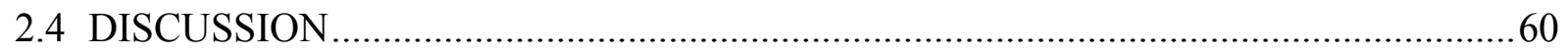

2.4.1 The hydrophilic-lipophilic nature of silicone oils and silicone alkyl polyether

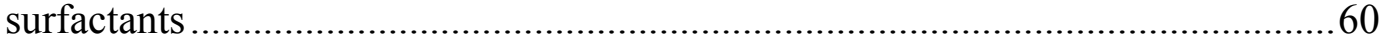

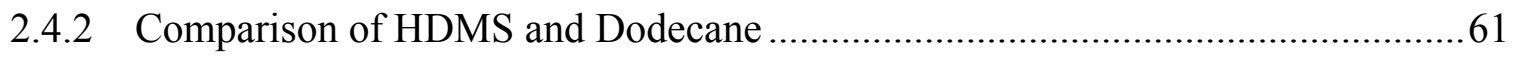

2.4.3 Validation of the HLD framework in literature .............................................62

2.4.4 Comparison of characteristic curvature and hydrophilic-lipophilic balance.........64

2.4.5 Phase Behaviour and Polymerization .........................................................67

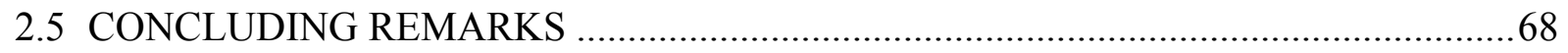


3 SOLUBILITY AND SOLUBILIZATION: EVALUATING PARAMETERS AND DEVIATIONS IN THE HLD FRAMEWORK ................................................................. 73

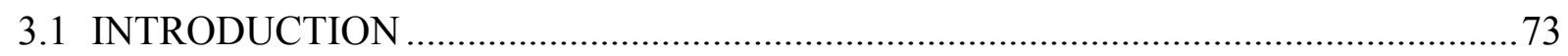

3.2 AQUEOUS MONOMERS AND THE LINKER EFFECT ........................................74

3.3 MONOMER SOLUBILITY IN AQUEOUS SOLUTION ..........................................76

3.3.1 Estimating $\varphi(A)$ for HEMA and MAA in aqueous solution ..............................77

3.3.2 Monomer solubility, phase separation and precipitation ................................... 78

3.3.3 Discussion: Solubility and the Solubilization............................................... 81

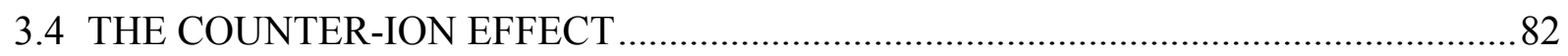

3.4.1 Phase behaviour variation with counter-ion size ......................................... 83

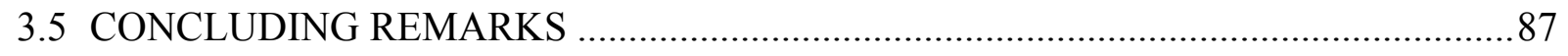

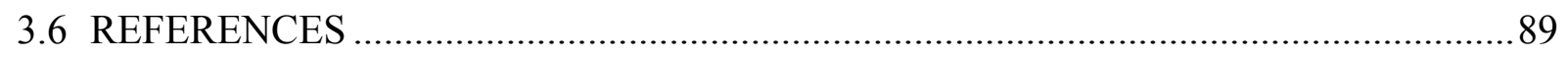

4 INTERPENETRATING POLYMER NETWORKS TEMPLATED ON BICONTINUOUS MICROEMULSIONS CONTAINING SILICONE OIL, METHACRYLIC ACID AND HYDROXYETHYL METHACRYLATE ...........................90

4.1 INTRODUCTION AND BACKGROUND ....................................................... 90

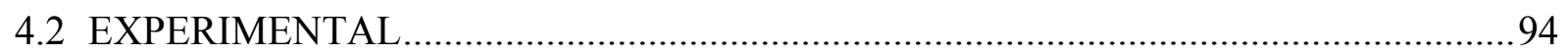

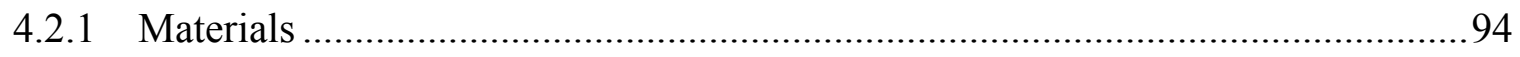

4.2.2 Microemulsion preparation....................................................................... 96

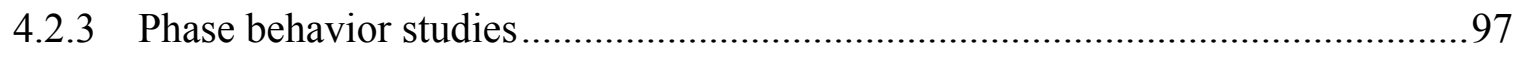

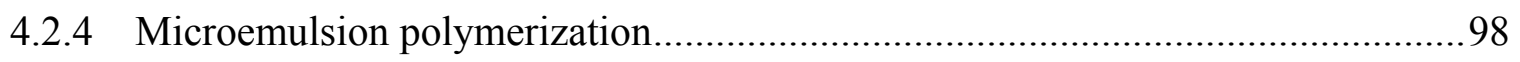

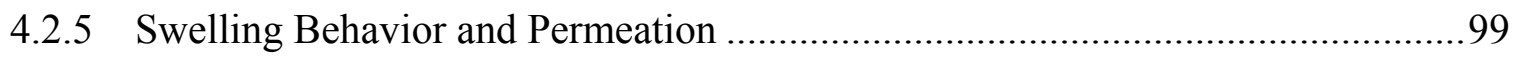

4.2.6 Laser Scanning Confocal Microscopy (LSCM) and Differential Scanning

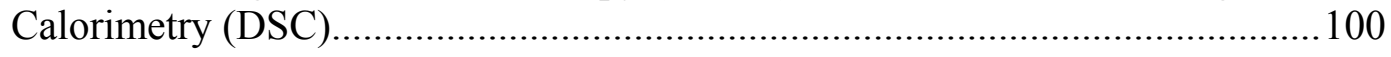

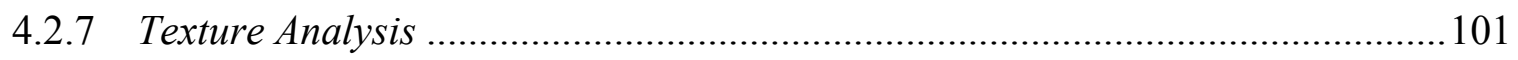

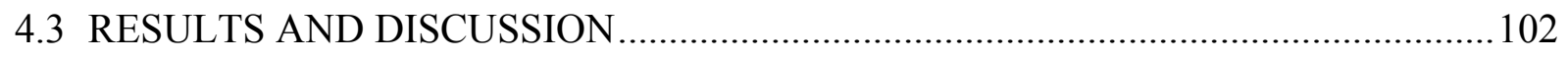

4.3.1 Phase Behavior of Reactive Silicone Microemulsions ..................................... 102 
4.3.2 Microemulsion Polymerization and LSCM............................................... 105

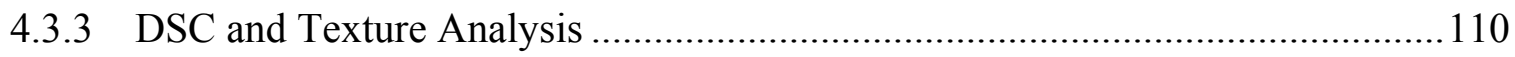

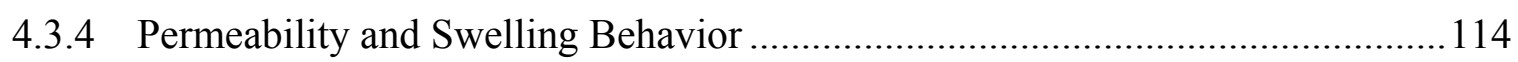

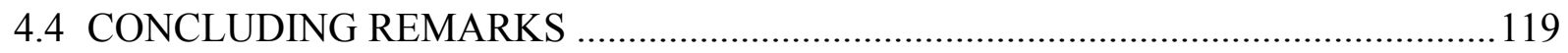

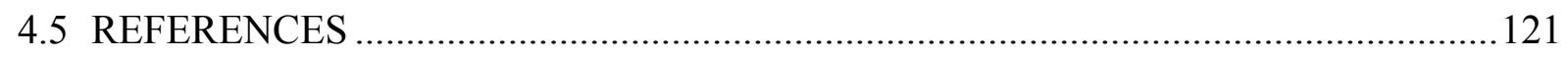

5 MICROEMULSION PHASE BEHAVIOUR AND POLYMER STRUCTURE ..................124

5.1 THE INFLUENCE OF MICROEMULSION MORPHOLOGY AND PHASE

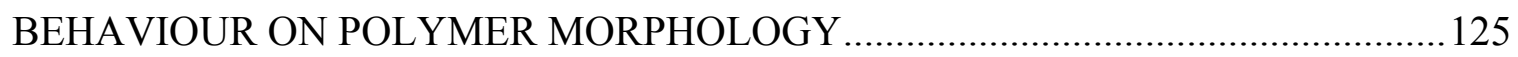

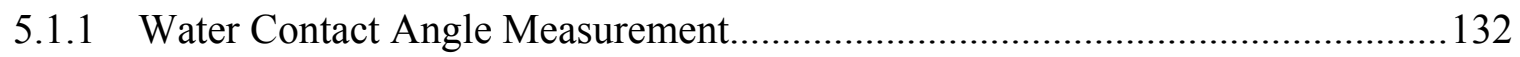

5.2 ON POTENTIAL LINKS BETWEEN PHASE BEHAVIOUR, POLYMER STRUCTURE, AND POLYMER PROPERTIES ................................................... 133

5.3 TRANSMISSION ELECTRON MICROSCOPY REVEALS NANOSCALE

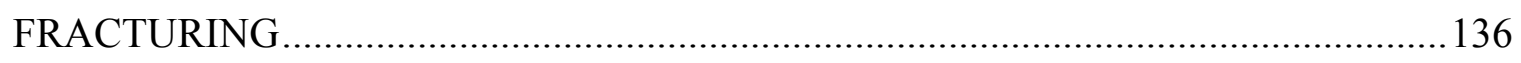

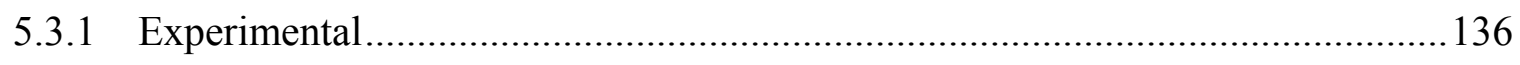

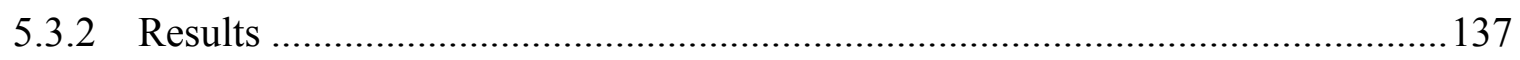

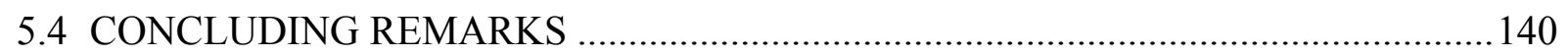

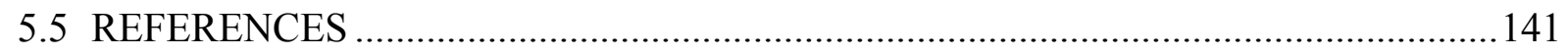

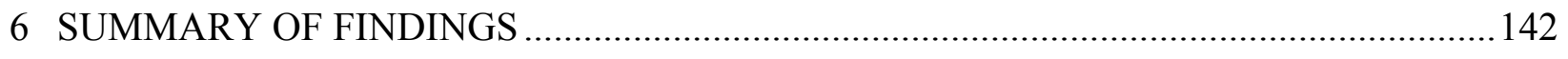

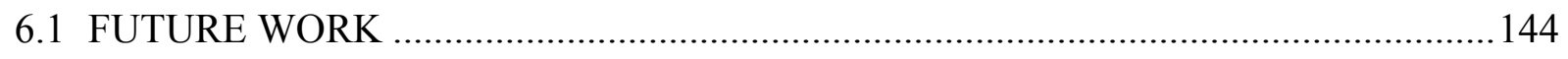

APPENDIX A: Surfactant and Oil Characteristic Values ................................................. 148

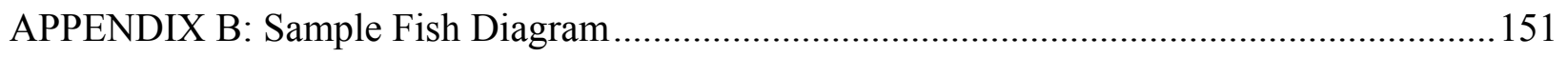




\section{List of Abbreviations and Symbols}

\begin{tabular}{|c|c|c|c|}
\hline Abbreviation & Meaning & Abbreviation & Meaning \\
\hline$A_{X Y}$ & $\begin{array}{l}\text { Interfacial Interactions between } \\
\text { Component } X \text { and } Y\end{array}$ & PIPS & $\begin{array}{l}\text { Polymerization Induced Phase } \\
\text { Separation }\end{array}$ \\
\hline$b$ & $\begin{array}{l}\text { Salinity Parameter }- \text { Nonionic } \\
\text { Surfactants }\end{array}$ & $S, S^{*}$ & Salinity, Optimal Salinity \\
\hline$c$ & $\begin{array}{l}\text { Temperature parameter - Nonionic } \\
\text { Surfactants }\end{array}$ & SANS & Small Angle Neutron Scattering \\
\hline$C$ & Concentration & $S A X S$ & Small Angle X-Ray Scattering \\
\hline$C_{c}$ & $\begin{array}{c}\text { Characteristic Curvature - Ionic } \\
\text { Surfactant }\end{array}$ & $S D$ & Spinodal Decomposition \\
\hline$C_{c n}$ & $\begin{array}{c}\text { Characteristic Curvature - Nonionic } \\
\text { Surfactant }\end{array}$ & $T$ & Temperature \\
\hline$D L S$ & Dynamic Light Scattering & TIPS & Thermally Induced Phase Separation \\
\hline$D S C$ & Dynamic Scanning Calorimetry & $U C S T$ & Upper Critical Solution Temperature \\
\hline$E A C N$ & (Equivalent) Alkane Carbon Number & $V_{i}, v_{i}$ & Volume of component $i$ \\
\hline$f(A)$ & $\begin{array}{l}\text { Co-surfactant function - Ionic } \\
\text { Surfactants }\end{array}$ & $Y_{i}$ & Mole fraction of component $i$ \\
\hline$H L B$ & Hydrophilic-Lipophilic Balance & $\alpha_{T}$ & $\begin{array}{l}\text { Temperature parameter - Ionic } \\
\text { Surfactants }\end{array}$ \\
\hline$H L D$ & Hydrophilic-Lipophilic Difference & $\gamma$ & Interfacial Tension (mathematical) \\
\hline$I F T$ & Interfacial Tension & $\Gamma$ & Surface Concentration \\
\hline$K$ & Equivalent Alkane parameter & $\Delta G_{m i x}$ & Free Energy of Mixing \\
\hline$k$ & Boltzmann constant & $\Delta H_{m i x}$ & Enthalpy of Mixing \\
\hline$L C S T$ & Lower Critical Solution Temperature & $\Delta S_{m i x}$ & Entropy of Mixing \\
\hline$L S C M$ & $\begin{array}{c}\text { Laser Scanning Confocal } \\
\text { Microscopy }\end{array}$ & $\mu E$ & Microemulsion \\
\hline$N$ & Avogadro's Number & $\varphi(A)$ & $\begin{array}{l}\text { Co-surfactant function - Nonionic } \\
\text { Surfactants }\end{array}$ \\
\hline$N G$ & Nucleation and Growth & & \\
\hline
\end{tabular}




\section{List of Tables}

Table 2-1: Summary of surfactant properties ${ }^{46}$

Table 2-2: Calculated $\mathrm{C}_{\mathrm{CN}}$ values for the $\mathrm{J} 208$ series of silicone alkyl polyether surfactants in systems containing $0.65 \mathrm{cSt}$ silicone oil and deionized water.

Table 2-3: Calculated HLD values for formulations containing 0.65 or $3 \mathrm{cSt}$ silicone oil, silicone alkyl polyether surfactant, and water with equivalent salinities of 0 and $14.54 \mathrm{~g} / 100 \mathrm{~mL}$. HLD values are calculated for a blend of surfactants $\mathrm{A}$ and B at $25^{\circ} \mathrm{C}$. 48

Table 2-4: Comparison of HDMS and Dodecane properties 62

Table 3-1: Selected formulation specifications for evaluation of the linker effect. Samples were formed from equal parts aqueous, oil and surfactant and equilibrated at $25^{\circ} \mathrm{C}$ for 14 days. Percentages are by volume. The surfactant blend contains equal parts surfactants A and B...... 75

Table 4-1: Summary of silicone surfactant properties ${ }^{34}$ 95 


\section{List of Figures}

Figure 1-1: Classification of polymers by molecular structure.

Figure 1-2: General phase behaviour for a polymer blend or solution with either an upper (UCST) or lower (LCST) critical solution temperature. 6

Figure 1-3: Schematic comparison of co-polymer formats

Figure 1-4: Schematic representation of sequential and simultaneous IPN synthesis.

Figure 1-5: Typical microemulsion structures and dependence on formulation variables. The persistence length is marked by $\xi$ for bicontinuous microemulsions.

Figure 1-6: Sample pseudo-ternary diagram. Microemulsion regions are marked by number of phases. Bars indicate location of microemulsion for biphasic regions. The three phase region contains a middle microemulsion phase.

Figure 1-7: Schematic representation of the linker effect. The use of co-surfactants and linkers extends the interfacial ordering of the surfactant deeper into each phase. The location and overlap of boxes is intended to reflect the relative distribution of each species.

Figure 1-8: Mass/ion transfer between w/o droplets.

Figure 1-9: Schematic depiction of oil, water and surfactant interactions

Figure 2-1: General structure of silicone alkyl polyether surfactant (PEG-8 Dimethicone). The

hydrophilicity is determined by the ratio of polyether to silicone and alkyl groups. ${ }^{46}$ 37

Figure 2-2: Salinity range required to form a three phase $\mu \mathrm{E}$ for mixtures of $0.65 \mathrm{cSt}$ silicone oil (A) or $3 \mathrm{cSt}$ silicone oil (B) with toluene as a function of toluene volume fraction in the oil blend. Dashed line represents the average value of $\ln \mathrm{S}^{*} . \mathrm{T}=20^{\circ} \mathrm{C}$

Figure 2-3: Salinity range required to form a three phase $\mu \mathrm{E}$ for mixtures of dodecane with toluene as a function of toluene volume fraction in the oil blend. Dashed line represents the average value of $\ln \mathrm{S}^{*} . \mathrm{T}=20^{\circ} \mathrm{C}$ 
Figure 2-4: Effect of acrylic monomers on phase behaviour. A) Phase scan of formulations containing equal parts oil and aqueous phase and a 50/50 blend of surfactants $\mathrm{A}$ and $\mathrm{B}$ with increasing MAA/HEMA content in aqueous solution (phase boundaries highlighted) B) Conductance measurement for formulations in $3 \mathrm{a}$. Formulations use $0.65 \mathrm{cSt}$ oil, and contain $10 \%$ surfactant by volume. $\mathrm{T}=25^{\circ} \mathrm{C}$.

Figure 2-5: Normalized electrical conductivity (conductivity of the microemulsion/ conductivity of aqueous phase) for formulations with and without HEMA/MAA (33\% and 22\% V/V in aqueous solution, respectively). Formulations contain a 1:1 ratio of $0.65 \mathrm{cSt}$ oil and aqueous solution, and contain $10 \%$ surfactant by volume. $\mathrm{T}=25^{\circ} \mathrm{C}$.

Figure 2-6: Temperature-dependent phase behaviour in $\mathrm{pH} 2.5$, deionized water based system (n $=3$ ). Each point represents one sample (10\% increments by volume), with higher resolution ( $2 \%$ increments) near phase boundaries.

Figure 2-7: Phase behaviour for $\mathrm{pH} 6$ systems adjusted with $\mathrm{NaOH}(\mathrm{n}=3)$. Each point represents one sample ( $10 \%$ increments by volume), with higher resolution ( $2 \%$ increments) near phase boundaries 55

Figure 2-8: Normalized conductance scans of $\mathrm{pH} 2.5$ (triangular points) and $\mathrm{pH} 6$ (square points, $\mathrm{NaOH}$ adjusted) systems. Conductance is normalized to the conductance of the aqueous solution used, and is measured at $25^{\circ} \mathrm{C}(\mathrm{n}=3)$. Conductance is reported for homogeneous formulations only

Figure 2-9: Viscosity as measured by falling ball viscometer $(\mathrm{n}=3)$ for $3 \mathrm{cSt}, \mathrm{pH} 6$ system, $\mathrm{T}=$ $25{ }^{\circ} \mathrm{C}$.

Figure 2-10: Comparison between the molar solubilization ratio of water in AOT-0.65 cSt microemulsions obtained experimentally by Binks et al ${ }^{22}$ and predicted using the HLD-NAC model 64

Figure 2-11: HLB vs. $C_{c n}$ for selected alkane alcohol ethoxylated $\left(C_{n} E_{j}\right)$, nonylphenol ethoxylated $\left(\mathrm{NPE}_{\mathrm{j}}\right)$ and silicone alkyl polyether surfactants (J208-X12). HLB and $\mathrm{C}_{\mathrm{CN}}$ values for alcohol ethoxylated and nonylphenol ethoxylated surfactants derived from ${ }^{48}$. 66 
Figure 3-1: Schematic representation of proposed linker effect in silicone microemulsion based on aqueous solubility/miscibility. The central line represents the oil-water interface. The locations of each box represent the theoretical overlap between molecules of each species near the interface, reflecting the extension of interfacial order into each phase.

Figure 3-2: Normalized conductance scans for microemulsion and excess aqueous phase in $\mathrm{pH}$ 2.5 formulation. Conductance is normalized to the conductance of the aqueous solution used, and is measured at $25{ }^{\circ} \mathrm{C}(\mathrm{n}=3)$. Points below $70 \%$ aqueous content are homogeneous microemulsions

Figure 3-3: Polymerization and settling of reactive monomer components and aggregation of surfactants with (Left) 60\% surfactant and 40\% pH 2.5 aqueous phase; (Middle) 10\% surfactant and $40 \%$ pH 2.5 aqueous phase; (Right) 40\% surfactant and 20\% pH 2.5 aqueous phase. 80

Figure 3-4: Phase behaviour for $\mathrm{pH} 6$ systems adjusted with $\mathrm{LiOH}$. Each point represents one sample (10\% increments by volume), with higher resolution ( $2 \%$ increments) near phase boundaries. Point of comparison at $35^{\circ} \mathrm{C}$ marked with black circle.

Figure 3-5: Phase behaviour for $\mathrm{pH} 6$ systems adjusted with $\mathrm{KOH}(\mathrm{n}=3)$. Each point represents one sample (10\% increments by volume), with higher resolution ( $2 \%$ increments) near phase boundaries. Point of comparison at $35^{\circ} \mathrm{C}$ marked with black circle.

Figure 3-6: Phase behaviour for $\mathrm{pH} 6$ systems adjusted with $\mathrm{CsOH}(\mathrm{n}=3)$. Each point represents one sample ( $10 \%$ increments by volume), with higher resolution ( $2 \%$ increments) near phase boundaries. Cesium precipitation occurred at $25^{\circ} \mathrm{C}$, thus the phase behaviour reflects only the liquid phases. Point of comparison at $35^{\circ} \mathrm{C}$ marked with black circle.

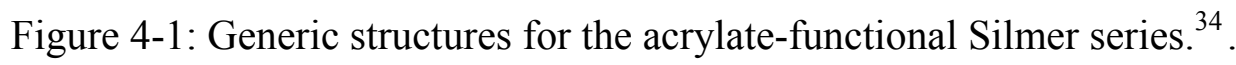
96

Figure 4-2: Phase behavior for formulations containing MAA and HEMA in aqueous solution at pH 6, vinyl and hydride terminated 3 cSt silicone oil, and a blend of non-reactive silicone lauryl polyether surfactants at $\mathrm{T}=25^{\circ} \mathrm{C}$ (left) and $\mathrm{T}=55^{\circ} \mathrm{C}$ (right). Each point represents one sample ( $10 \%$ increments by volume) with higher resolution ( $5 \%$ increments, not marked) near phase boundaries. 
Figure 4-3: Phase behavior of formulations containing MAA and HEMA in aqueous solution at $\mathrm{pH}$ 6, vinyl and hydride terminated $3 \mathrm{cSt}$ silicone oil, and silicone acrylate surfactant $\mathrm{C}$ (left) or $\mathrm{D}$ (right) at $\mathrm{T}=25,35,45$ and $55^{\circ} \mathrm{C}$. Phase behavior was observed to be unchanged over the range of temperatures investigated. Each point represents one sample (10\% increments by volume), with higher resolution ( $5 \%$ increments, not marked) near phase boundaries. 103

Figure 4-4: Comparison of hydrated IPNs at 5\% v/v TEGDMA with hydrated P(MAA-HEMA) and PDMS components used in their formulation.

Figure 4-5: Selected LSCM images of NR-IPN at equilibrium swelling, 5\% v/v TEGDMA. Images proceed from left to right and top to bottom in $2.5 \mu \mathrm{m}$ increments. The top left image represents the glass-contacting surface, and the bottom right image represents a depth of $20 \mu \mathrm{m}$. Scale bars are $2 \mu \mathrm{m}$. 107

Figure 4-6: Average domain size depth profile for NR-IPN at equilibrium swelling, 5\% v/v TEGDMA. 108

Figure 4-7: LSCM image of C-IPN (left) and D-IPN (right) at equilibrium swelling, 5\% v/v TEGDMA, $10 \mu \mathrm{m}$ depth. 110

Figure 4-8: Sample DSC comparison of an NR-IPN (5\% v/v TEGDMA) with the PDMS and $\mathrm{P}(\mathrm{MAA}-\mathrm{HEMA})(5 \% \mathrm{v} / \mathrm{v}$ TEGDMA) components used in its formulation. DSC measurements are taken on dry samples.

Figure 4-9: Texture analysis of microemulsion templated IPNs with 3\% v/v TEGDMA. A) Force of compression/penetration through IPNs as a function of depth. B) Comparison of peak forces with control PDMS and P(MAA-HEMA), 3\% v/v TEGDMA. Peak forces are normalized to the peak force of compression/penetration for the PDMS control. 113

Figure 4-10: Swelling behavior for polymers formed from silicone microemulsions with nonreactive surfactants at 3\%,5\% and 10\% v/v (HEMA/MAA) TEDGMA. $\mathrm{N}=4$. Hydration is calculated as water mass/total swollen mass. Control hydrogel is polymerized from the aqueous mixtures used in the microemulsion-templated IPN. 115 
Figure 4-11: Comparison of maximum hydration for IPNs formed from formulations with and without polymerizable surfactant at 3\% and 10\% v/v (HEMA, MAA) TEGMA. $\mathrm{N}=4$. Control hydrogel is polymerized from the aqueous mixtures used in the microemulsion-templated IPN.

Figure 4-12: Comparison of vitamin $\mathrm{B}_{12}$ permeabilities $(\mathrm{n}=4)$ for IPNs at $3 \% \mathrm{v} / \mathrm{v}$ TEGDMA with PDMS-PMAA (Turner et al. ${ }^{50}, 30 \%$ PMAA) and estimates for the diffusion coefficient of $\mathrm{B}_{12}$ in water ${ }^{51-53}$

Figure 5-1 LSCM image of hydrogel-PDMS boundary in 0:5:5 formulation. The highlighted regions indicate the presence of sodium fluorescein, $15 \mu \mathrm{m}$ depth.

Figure 5-2: LSCM image of 3:2:5 NR-IPN swollen in sodium fluorescein solution, $15 \mu \mathrm{m}$ depth.

Figure 5-3: Comparison of materials polymerized from microemulsion (lower phase) and excess oil (upper phase) in 3:5:2 NR formulation.

Figure 5-4: LSCM image of 3:5:2 NR- Polymer blend swollen in sodium fluorescein solution, $15 \mu \mathrm{m}$ depth. This formulation begins as a lower phase microemulsion at $25^{\circ} \mathrm{C}$, and the material shown here is extracted from that region. 130

Figure 5-5: LSCM image of 2:1:1 NR-IPN swollen in sodium fluorescein solution, $15 \mu \mathrm{m}$ depth.

Figure 5-6: Schematic representation of surfactant removal and replacement with free water in NR-IPN, and polymer expansion/hydration/relaxation.

Figure 5-7: NR-IPN swelling comparison at 3\% and 10\% TEDGMA. Approximate swelling space generated by surfactant removal denoted by solid line (3:2:5 NR formulation) and dashed line (2:1:1 NR formulation). 135

Figure 5-8: TEM images of dry NR-IPN, 5\% v/v TEGDMA. Darkened areas are associated with higher silicone content. ${ }^{9}$ A) IPN nanostructure is heterogeneous, but contains gaps. B) Fracturing and interpenetration at higher magnification. 139 


\section{List of Equations}

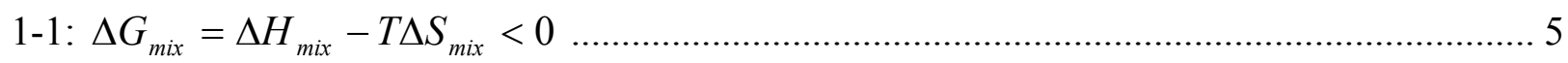

$1-2: \frac{\partial^{2} \Delta G_{m i x}}{\partial \varphi^{2}}>0$

1-3: $\Delta G_{m i x}=k T V\left[\frac{\varphi_{1}}{v_{1}} \ln \varphi_{1}+\frac{\varphi_{2}}{v_{2}} \ln \varphi_{2}+\frac{\chi_{12}}{v_{s}} \varphi_{1} \varphi_{2}\right] \ldots$

1-4: $\Delta G=\Delta H-T \Delta S+\gamma \Delta A$

1-5: $\gamma=\left(\frac{\delta G}{\delta A}\right)_{T, P}$

1-6: $\xi=Z \times\left(\frac{\varphi_{\text {oil }} \varphi_{\text {aqueous }}}{C_{s} A_{s}}\right)$

1-7: $\Gamma=\frac{1}{2.303 R T}\left(\frac{\partial \gamma}{\partial \log C}\right)_{T}$

1-8: $A_{s}=\frac{10^{16}}{N \times \Gamma}$

1-9: $R=\frac{A_{S O}-A_{o o}-A_{L L}}{A_{S W}-A_{W W}-A_{H H}}$

1-10: $H L B=20 \times \frac{M_{h}}{M_{t}}$

1-11: $\mathrm{HLD}=\ln (S)-K^{*} E A C N-f(A)-\alpha \Delta T+C_{c}$

1-12: $\mathrm{HLD}=b(S)-K * E A C N-\varphi(A)+c \Delta T+C_{c n}$

2-1: $\mathrm{HLD}=\ln (S)-K^{*} E A C N-f(A)-\alpha \Delta T+C_{c}$ 
2-2: $\mathrm{HLD}=b(S)-K * E A C N-\varphi(A)+c \Delta T+C_{c n}$

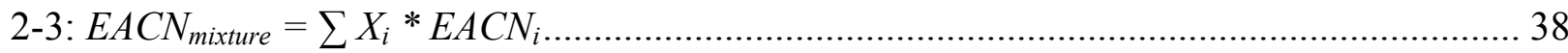

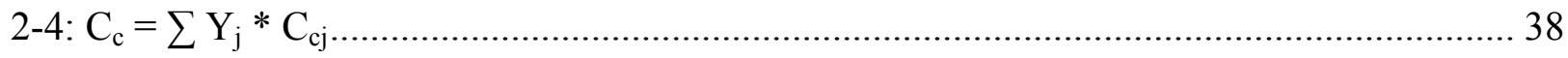

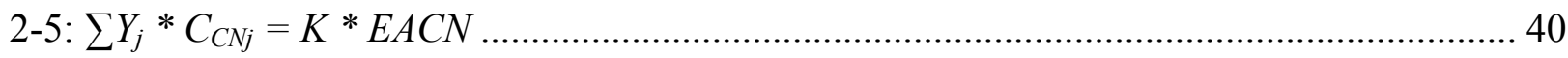

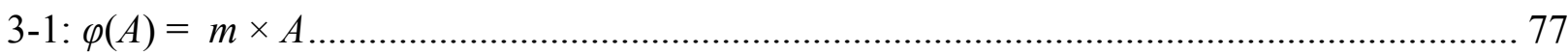

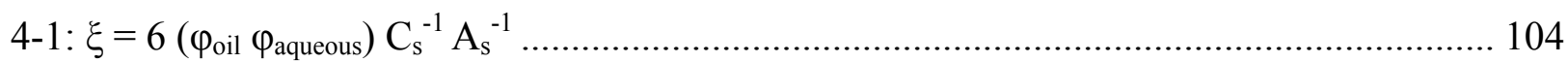




\section{INTRODUCTION}

The widespread availability of polymeric materials with distinct physical and chemical properties offers a number of opportunities for generating novel materials. By combining two or more polymers with known properties, we are able to design materials that combine the characteristics of each polymer. The combination can be synergistic, where the blended material possesses more desirable temperature stability, solvent resistance, or mechanical properties than could be obtained through an individual polymer. The combination can also apply the properties of one polymer to address the deficiencies in another. Approaching the development of new materials through alloys, blends and co-polymers has become a commercially important area of polymer science, as it is often both easier and cheaper to use existing materials than it is to design and synthesize entirely new polymers. ${ }^{1-3}$

The different types of polymer blends can be characterized by their phase behaviour, which in turn is based on polymer miscibility ${ }^{4}$. There are a number of methods for combining polymers, with an important focus on compatibilization. The simplest methods involve physical blending through melt-mixing and shear, or dissolving two compatible materials in solvent. The result is often a macroscopic mixture with significant phase separation. More sophisticated physicochemical approaches may involve the use of co-polymerization, interfacial agents (i.e. polymeric surfactants), or interpenetrating crosslinks. ${ }^{1-5}$ 
Designing polymer blends is particularly important in the field of biomaterials, where using existing, approved materials is generally more efficient and cost effective than designing and certifying novel materials. Useful polymer blends can fall into several categories. Common applications include elastomer blends for high endurance materials, emulsified blends for adhesives and coatings and hydrophilic-rubber blends for biomaterials and multiphase transport requirements. In particular, many blends focus on lending the mechanical strength of one material to another material that may have desirable chemical properties ${ }^{4}$. Of particular interest is combining the aqueous transport of hydrogels with the family of polydimethylsiloxanes (PDMS) and their derivatives. The important features of PDMS, such as chain mobility, organization and density of methyl groups and the Si-O backbone, yield desirable physical properties and chemical stability for a wide range of applications. Crosslinked PDMS elastomers are useful as oxygen permeable biomaterials or as substrates for microfluidic devices ${ }^{3,6-9}$. In order to develop processes for combining distinct polymers, it is important to understand and resolve the miscibility gap. While the thermodynamics of polymer solutions and blends are well understood ${ }^{1,2}$, it remains important to investigate and refine the processes by which polymer miscibility can be achieved. 


\subsection{COMBINING POLYMERS}

\subsubsection{Polymer Basics}

The properties of a polymer are strongly dependent on the properties of the monomer, as well as the arrangement of polymer chains. Chain length and molecular weight will also impact polymer properties, though with diminishing returns. Various methods for polymer classification are available. ${ }^{1-4}$ Early classification proceeded according to heat-dependent behaviour, dividing polymers into two categories: Thermoplastics and thermosets. Thermoplastics are distinguished by reversible structural changes in response to heating and cooling cycles. Once cured, thermosets degrade upon heating. In the modern approach, polymers are classified according to their characteristic polymerization reaction. Chain growth polymerization is defined by the sequential addition of unsaturated monomer to the end of a growing polymer chain. In step growth polymerization, the functional monomers combine step-wise into increasingly larger units. A third method of classification focuses on the molecular structure. Linear chain polymers consist of long, regular chains of covalently bonded repeat units. Interactions between chains can be overcome by an increase in thermal energy without destroying the chain, leading to reversible structural changes in response to a heating cycle. If the chain interactions are strong and well defined, the polymer can form a crystalline structure. Branched polymers contain side chains attached to the backbone chain, introducing irregularities to the structure. Branching usually requires that some component of the polymer possess more than two functional groups, though it also commonly occurs as a result of chain transfer reactions in radical polymerization. At high levels of branching, it is possible for side chains to form connections between backbone 
chains, resulting in the third classification: Network polymers. Network polymers are of particular interest, as they form a three-dimensional structure stabilized by the permanent crosslinks. These classifications are depicted in Figure 1-1. ${ }^{1,2}$

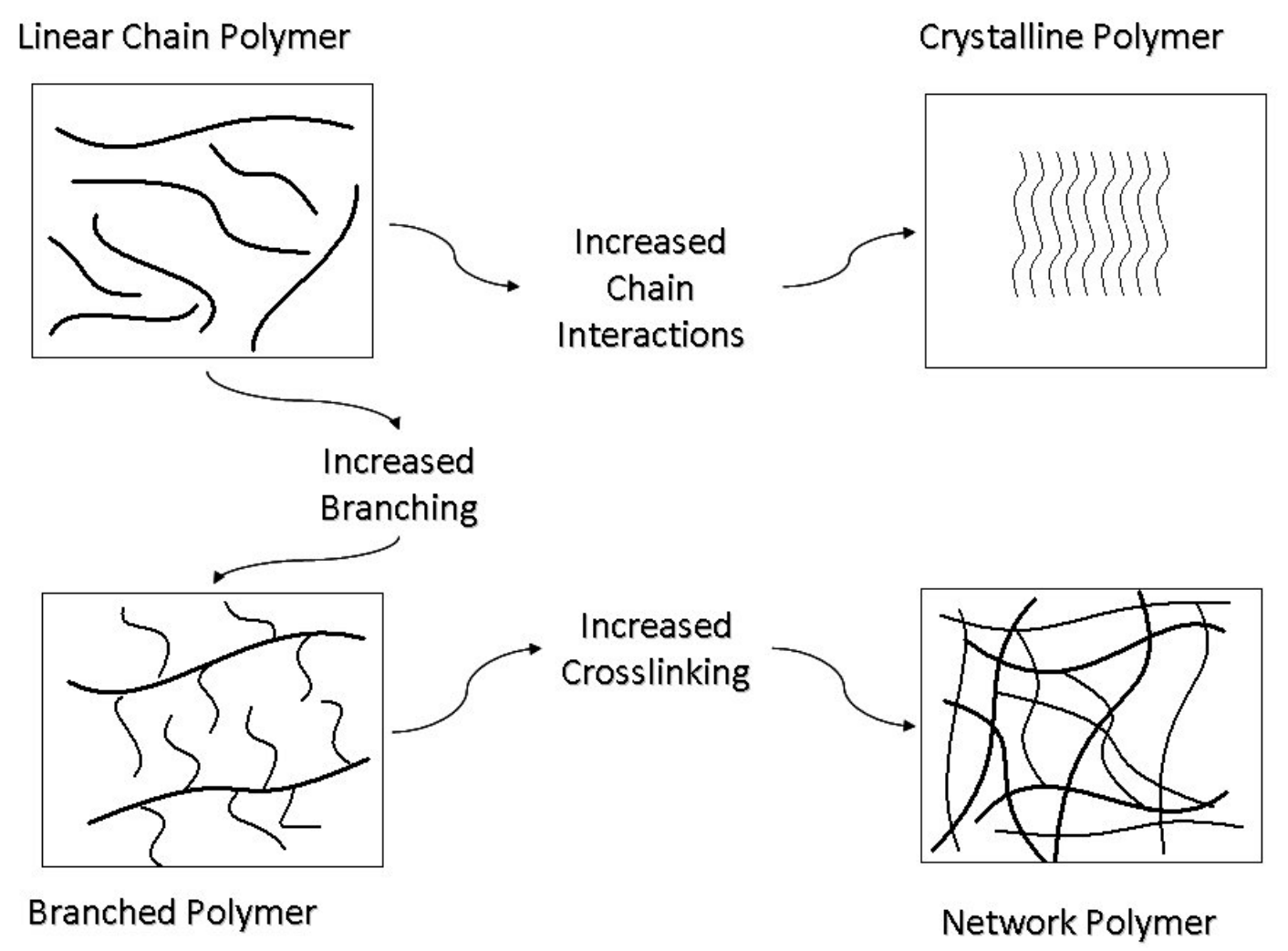

Figure 1-1: Classification of polymers by molecular structure.

\subsubsection{Thermodynamics and Phase Behaviour}

The thermodynamics of polymer solutions are governed by the Gibbs free energy of mixing, $\Delta \mathrm{G}_{\text {mix }}$, the enthalpy of mixing, $\Delta \mathrm{H}_{\text {mix }}$ and the entropy of mixing, $\Delta \mathrm{S}_{\text {mix }}$. Whether dissolving a 
polymer in solvent or creating a mixture of two or more polymers at constant temperature $(T)$ and pressure $(P)$, the Gibbs free energy must follow the relation:

1-1: $\Delta G_{m i x}=\Delta H_{m i x}-T \Delta S_{m i x}<0$

Thus a stable mixture is obtained when the free energy of the mixture is less than the combined free energies of the components. The concept of stability refers to three possible states: Stable, metastable and unstable. The metastable regions correspond to local or weak minima in the free energy of the system. Unstable formulations decompose into multiple phases, as the free energy of mixing is positive. ${ }^{1,10}$ In addition to minimizing free energy, the conditions for stability are satisfied when:

1-2: $\frac{\partial^{2} \Delta G_{m i x}}{\partial \varphi^{2}}>0$

Where $\varphi$ denotes the mole fraction of given component in a theoretical two-component system. Examples of these regions and boundaries are illustrated in Figure 1-2. The binodal curve marks the boundary between the stable and metastable region. Within the binodal curve, phase separation is favored. The spinodal marks the boundary between the metastable and unstable region, satisfying the condition, where $\frac{\partial^{2} \Delta G_{m i x}}{\partial \varphi^{2}}=0$. The upper (UCST) and lower (LCST) critical solutions temperature mark the highest and lowest temperatures that define the transition from instability to stability. A solvent-polymer solution will typically exhibit a UCST, though there are examples of systems exhibiting an LCST, such as poly(N-isopropylacrylamide), NIPAAM, in water. A polymer-polymer mixture may exhibit an LCST, UCST, or both. Systems exhibiting a UCST are associated with positive entropy of mixing, while systems exhibiting an LCST are associated with negative entropy of mixing. ${ }^{1,10}$ 


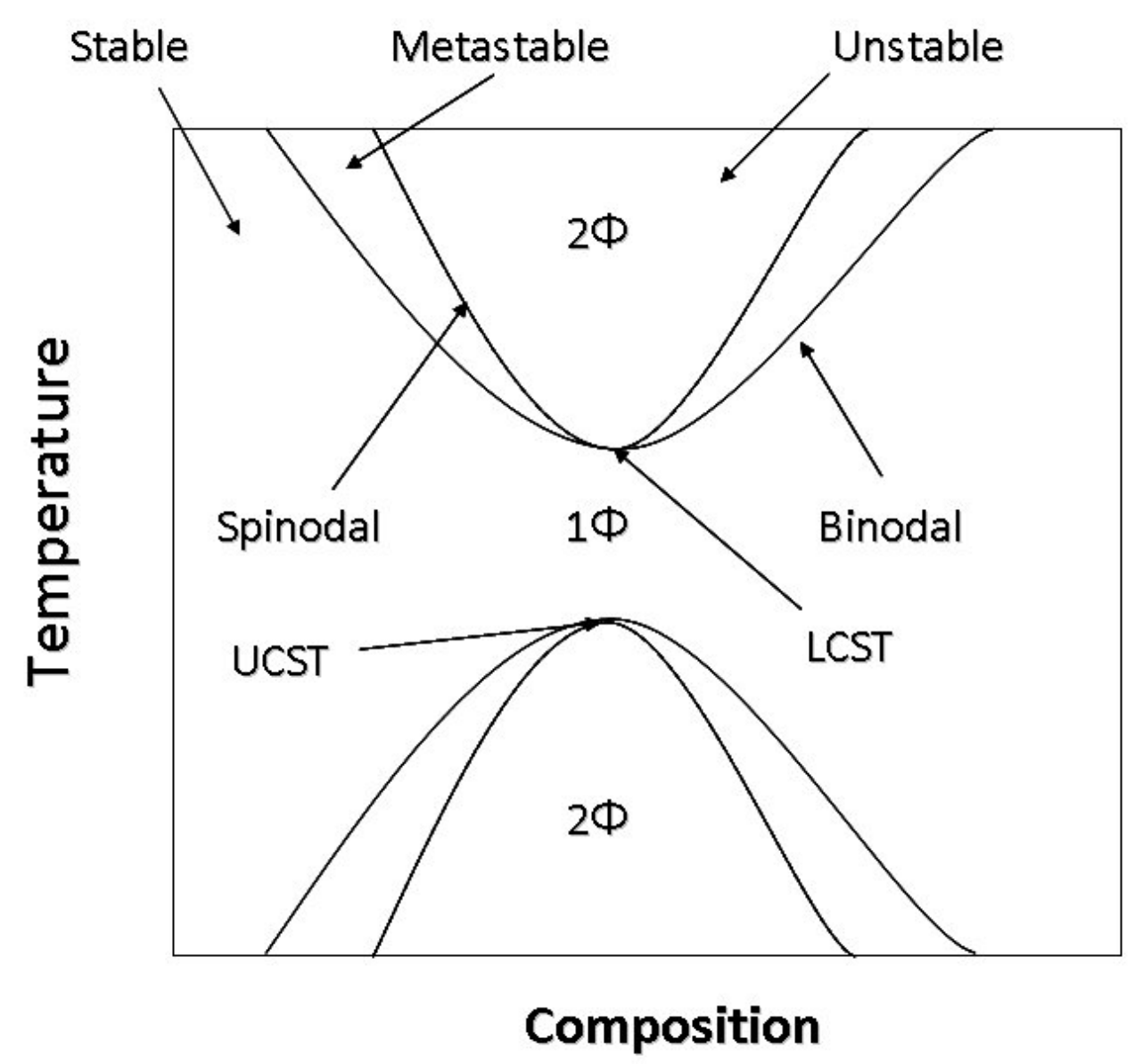

Figure 1-2: General phase behaviour for a polymer blend or solution with either an upper (UCST) or lower (LCST) critical solution temperature.

According to the Flory-Huggins theory modified for polymer mixtures ${ }^{1,10-12}$, the free energy of mixing for two polymers can be described as follows:

1-3: $\Delta G_{m i x}=k T V\left[\frac{\varphi_{1}}{v_{1}} \ln \varphi_{1}+\frac{\varphi_{2}}{v_{2}} \ln \varphi_{2}+\frac{\chi_{12}}{v_{s}} \varphi_{1} \varphi_{2}\right]$

where $k$ is the Boltzmann constant, $\mathrm{T}$ is temperature, $\mathrm{V}$ is the total volume, $\varphi_{\mathrm{i}}$ and $\mathrm{v}_{\mathrm{i}}$ are the volume fraction and volume per molecule of polymer (or solvent) $i$, respectively, $\mathrm{v}_{\mathrm{s}}$ is the 
interacting volume per lattice site in the Flory-Huggins model and $\chi_{12}$ is the Flory-Huggins interaction parameter. The first two terms correspond to the entropy of mixing, $\Delta \mathrm{S}_{\mathrm{mix}}$, while the last term corresponds to the enthalpy of mixing, $\Delta \mathrm{H}_{\text {mix }}$. Thus, if the volumes per molecule for the polymers are significantly larger than $\mathrm{v}_{\mathrm{s}}$, then this approach implies that $\Delta \mathrm{G}_{\text {mix }} \approx \Delta \mathrm{H}_{\text {mix }}{ }^{1}$

\subsubsection{Copolymers and Blends}

Overcoming the polymer miscibility gap does not necessarily require intimate mixing at the molecular scale. Instead, efforts to increase miscibility are generally focused on achieving blend properties (e.g. transition temperatures) that reflect a single material ${ }^{4}$. Mixtures of polymers can be characterized according to the interactions that resist the thermodynamically driven phase separation described above. In the case of mechanically mixed immiscible polymers, the interactions are limited to weaker secondary forces (Van der Waals). One common approach to stabilizing these blends is the introduction of an interfacial agent to reduce the surface tension between the primary polymers, such as a co- polymer or surfactant ${ }^{3,4}$. At the other end of the spectrum, two polymers can be held together by strong covalent bonds (ethylene vinyl acetate), ionic interactions (sodium alginate with calcium chloride), or physical entanglements (silicone hydrogel interpenetrating polymer networks) .

Lipatov et al. ${ }^{10}$ defines polymer mixtures associated with weaker interactions according to two categories: Alloys and blends. Alloys contain polymers that are miscible in the molten state, with structure dependent on thermodynamics and kinetics of cooling. A system with a UCST 
will phase separate on cooling, so that the final structure will depend on the balance between the kinetics of phase separation and the increasing viscosity. In a system with an LCST, the onephase structure is preserved on cooling. Blends are immiscible in the molten state. Thus, the molten mixing state would occur below the binodal for a system with UCST, and above the binodal for a system with LCST. Again, the structures are dependent on the degree of mixing and rate of phase separation. The temperature-phase behaviour relationship that characterizes these systems is referred to as thermally induced phase separation, or TIPS ${ }^{4,5,13}$.

Random copolymerization is an effective approach to combining the properties of multiple polymers, but is limited to polymers (or monomers) with similar solubility, or which require similar reaction environments. Other forms of copolymerization, such as block of graft, can be carried out sequentially in separate reaction environments. Covalent bonding is generally sufficient to prevent phase separation if the monomer units are intimately mixed. However, copolymers that contain large segments of a single type (i.e. block or graft copolymers) can still contain microphases as the segments phase separate. The type of copolymer that can be produced is dependent on the miscibility or common solubility of the two (or more) polymers being combined, and on the relative amounts and reaction rates of each component ${ }^{14}$. Several types of copolymers are depicted in Figure 1-3. 


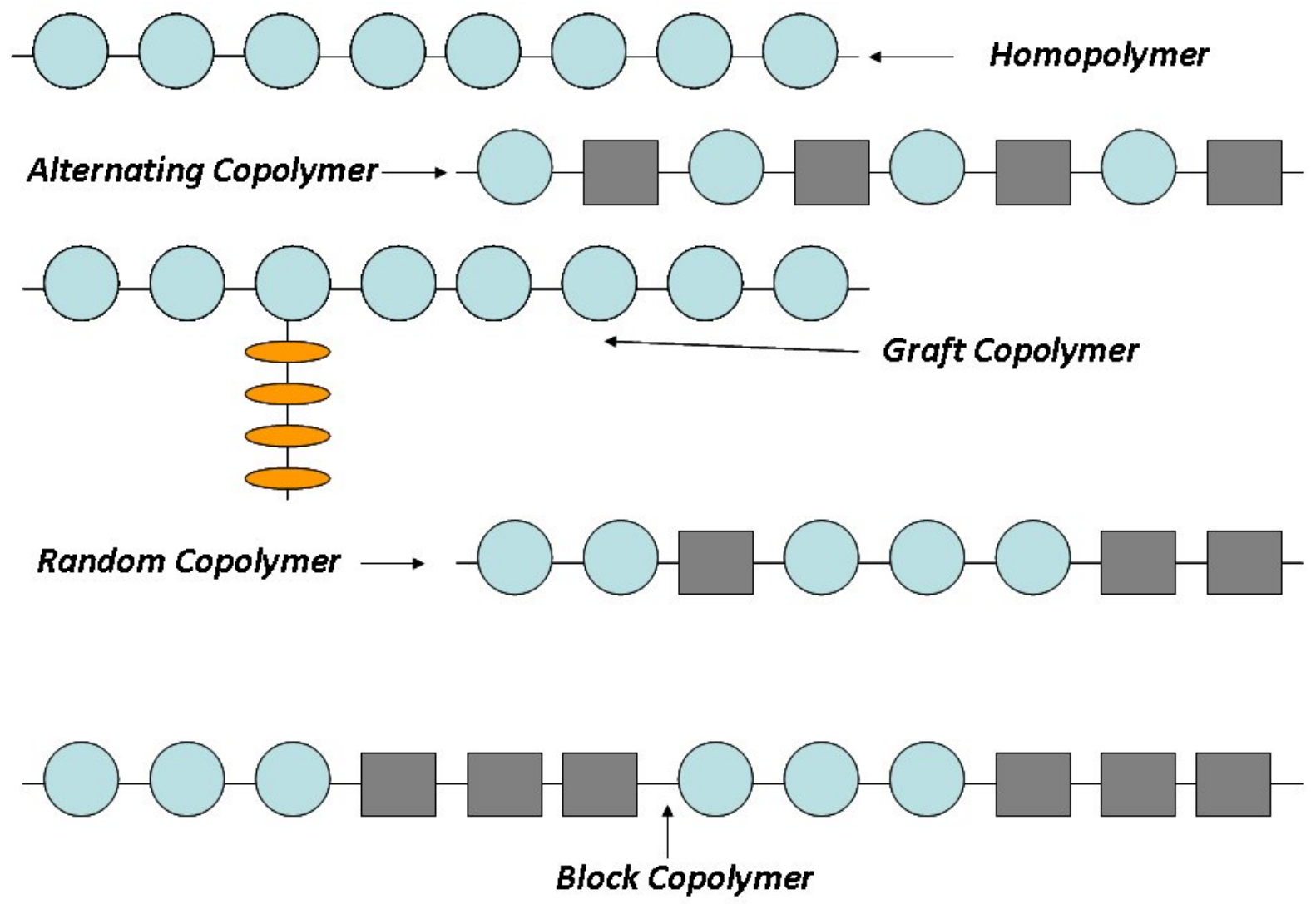

Figure 1-3: Schematic comparison of co-polymer formats

\subsubsection{Interpenetrating Polymer Networks}

The conventional model for interpenetrating polymer networks (IPNs) describes these as formed from two or more crosslinked polymer networks that are physically entangled but not covalently linked. The interpenetration ideally occurs on the molecular scale; however, the mixing of high molecular weight polymers on the molecular scale is hindered by the enthalpy of mixing, $\Delta \mathrm{H}_{\mathrm{mix}}$, 
as previously described. Thus phase separation becomes particularly important as polymerization proceeds, and this has been linked to IPN morphology. ${ }^{3,6,15-17}$

IPNs can be formed through sequential or simultaneous polymerization, depicted schematically in Figure 1-4. For simultaneous polymerization, the monomers, crosslinkers and activating agents for each component are pre-mixed. Ideally, the polymerization time scale is similar for all components. The process is defined by three critical steps: Gelation of network A, gelation of network $\mathrm{B}$, and phase separation. The resulting morphology is partly defined by the relative rates and the order in which the stages progress. For example, rapid, near-simultaneous polymerization of both networks will generally yield small domain sizes. Conversely, if phase separation occurs faster than gelation, then domain sizes will tend to be larger ${ }^{10,15,17}$. Simultaneous polymerization is limited to formulations with non-interfering reactions, and generally is only applicable to polymers that have some degree of miscibility or co-solubility. Sequential polymerization is primarily used to combine materials requiring incompatible polymerization environments or techniques. The "host" polymer is prepared first, and is then swollen with "guest" monomers and crosslinkers. The result is that the host polymer is likely to form the continuous phase, and the structure of the guest monomer is constrained by the high viscosity polymerization environment. Through both processes, the two phases form permanent physical entanglements that present a kinetic barrier to phase separation., $3,10,16,17$ 


\section{Sequential Polymerization}
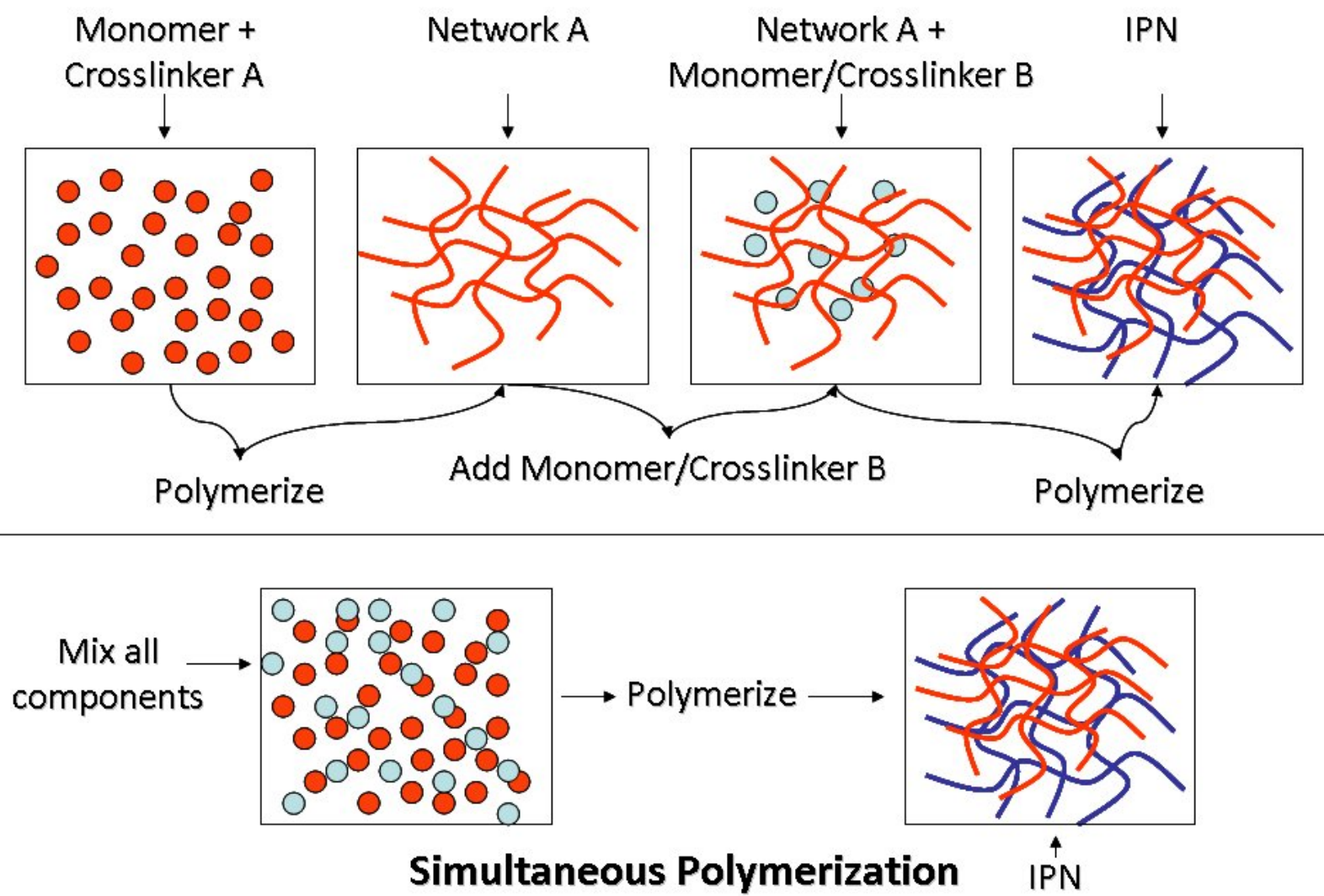

Figure 1-4: Schematic representation of sequential and simultaneous IPN synthesis.

In contrast to the TIPS that characterizes melt-mix blends, or the strong interactions of copolymerization, phase separation in IPNs is primarily defined by polymerization-induced phase separation, or PIPS ${ }^{6,18,19}$. The phase separation in IPNs can proceed through two mechanisms: Nucleation and growth (NG) or spinodal decomposition (SD). Nucleation and growth occurs in the metastable region of the phase diagram. Compositional fluctuations lead to the nucleation of a new phase. As fluctuations progress, nuclei grow and coalesce, leading to the formation of an irregular second phase. SD is the more common mechanism defining IPN phase behaviour and structure ${ }^{6,10,15}$. SD occurs in the unstable region of the phase diagram, and is 
marked by the formation of regular, periodic concentration fluctuations. These fluctuations in turn lead to the formation of an interpenetrating, two-phase structure. As the amplitude and wavelength of the fluctuations increases and phase separation progresses, there is a corresponding increase in the size distribution that eventually leads to nucleation, growth and coalescence. IPN morphology is thus dependent on both the thermodynamically driven phase separation, and the kinetic barrier to phase separation presented by the permanent crosslinking and accompanying increase in viscosity. ${ }^{10,18-23}$

\subsection{MICROEMULSIONS}

In its simplest form, a microemulsion $(\mu \mathrm{E})$ is a thermodynamically stable, macroscopically homogeneous mixture of a polar solvent (usually water), a non-polar solvent (oil) and an amphiphile (surfactant) with low interfacial tension (IFT). The concept of a surfactantcontaining system as a microemulsion was evolved by Hoar and Schulman ${ }^{24}$ in 1943 in reference to micellar systems and studies of oil solubilization at the time. Despite the framework introduced by Hoar and Schulman ${ }^{24}$ and later Winsor ${ }^{25,26}$, the original contributions by Sjoebloom and Friberg ${ }^{27}$ and Ekwall ${ }^{28}$ investigating micellar/inverse micellar systems, selfassembly and water-surfactant interactions were not initially considered microemulsions.

Gillberg et al. ${ }^{29}$ found that the inverse micellar systems could solubilize large amounts of oil, thus connecting these investigations to the modern understanding of microemulsions ${ }^{30}$. Danielsson and Lindman ${ }^{31}$ later presented their widely accepted definition of microemulsions in 1981, that: "A microemulsion is a system of water, oil, and amphiphile, which is a single optically isotropic and thermodynamically stable liquid solution." While sharing many 
important structural and behavioural properties, microemulsions are fundamentally different from conventional, or macro-emulsions. Terms such as emulsion, miniemulsion or nanoemulsion refer to oil-water dispersions that are kinetically stable, but not thermodynamically stable. In other words, the phase separated state is the equilibrium state, but the presence of a surfactant film presents a kinetic barrier to coalescence ${ }^{30,32-34}$.

Microemulsions should also be distinguished from molecular solutions, where the components are intimately mixed at the molecular level.

With the potential for high solubilization of hydrophilic and lipophilic compounds, thermodynamic stability and ease of formulation, the study of microemulsion phase behaviour and structure has recently re-emerged as a theme of interest in cosmetics, food and pharmaceuticals $^{32,35-38}$, fabric treatments ${ }^{39-42}$, and even as a platform for nanoscale polymerization ${ }^{43-47}$. Microemulsions allow for submicron mixing (1-100 nm) of otherwise immiscible polar and non-polar solvents through a surfactant mediated reduction in interfacial tension, and can form a variety of droplet, interpenetrating and parallel structures with different interfacial curvatures. ${ }^{33,48-51}$ However, designing microemulsion systems in order to exploit their low interfacial tensions requires an understanding of the formulation variables, since microemulsion morphology is linked to phase behaviour ${ }^{32,52,53}$. 


\subsubsection{Thermodynamics of Surfactant Systems}

The study of surfactant self-assembly systems could be considered as a precursor to the current field of microemulsions. Understanding the behaviour of surfactant self-assembly systems and interfacial tension becomes especially important as the interfacial area of a system increases relative to its volume, as is the case with high solubilization microemulsions. Surfactants can be divided into four groups: cationic, anionic, zwitterionic or nonionic. More simply, they can be considered as ionic or nonionic. Surfactants are amphiphiles, with a structure that includes both hydrophilic (head) and lipophilic (tail) groups. When a surfactant is introduced into a solvent, one of these components will disrupt the solvent structure. Thus in order to minimize the free energy of mixing, the surfactant will tend to concentrate at a polar - non-polar interface, ejecting the hydrophobic end from a polar solvent, or the hydrophilic end from a non-polar solvent ${ }^{51,53,54}$.

The thermodynamic basis for microemulsion formation is not yet as rigorously studied as that for polymer solutions, which may be a result of the difficulty in linking the wide range of complex, empirically studied surfactant systems to the fundamental processes that govern them

${ }^{52}$. However, critical areas such as droplet formation and questions of stability have been well studied ${ }^{53-55}$. The free energy of droplet formation differs slightly from that studied previously for polymer solutions. Instead of molecular mixing, we must consider the work required to form an interface of area $A$, with: 
1-4: $\Delta G=\Delta H-T \Delta S+\gamma \Delta A$

and

1-5: $\gamma=\left(\frac{\delta G}{\delta A}\right)_{T, P}$

Where $\gamma$ is the interfacial tension (IFT). If the initial state consists of two entirely phase separated liquids, it is expected that dispersion of one phase into another will increase the entropy, particularly as droplet size is decreased ${ }^{53-55}$. However, the immiscibility of oil and water is characterized by a high IFT, $\gamma$, so that the increase in interfacial area will correspond to an increase in Gibbs free energy ${ }^{56,57}$. Briefly, the introduction of a surfactant and co-surfactant at the interface lowers the interfacial tension, essentially replacing the higher energy water-oil interactions with lower energy water-amphiphile and oil-amphiphile interactions. While this reduction in the miscibility gap does not lead to a molecular solution, the low interfacial tension characteristic of microemulsions does lead to nanoscale mixing.

\subsubsection{Microemulsion Structure and Behaviour}

The low interfacial tensions and corresponding dispersion seen in microemulsions can lead to several kinds of structures. Microemulsions are typically seen as oil in water $(\mathrm{o} / \mathrm{w}$, Winsor type I) micelles, water in oil (w/o, Winsor type II) reverse micelles, or bicontinuous. (Winsor type III, IV) as defined by Winsor et al. ${ }^{25,26}$. Type I microemulsions consist of a surfactant-rich aqueous phase in equilibrium with excess oil and type II consists of a surfactant-rich oil phase in equilibrium with excess aqueous phase. Type III microemulsions contain a surfactant rich middle phase in equilibrium with both excess oil and aqueous phase ${ }^{58}$, while type IV 
microemulsions are macroscopically homogeneous, with no excess. The structure and transitions between these systems are depicted in Figure 1-5. The phase behaviour can be illustrated using a pseudo-ternary phase diagram, which may also include additional colloidal, self-assembled surfactant systems ${ }^{59}$, shown in Figure 1-6. The term "pseudo-ternary" refers to the possibility that one or more vertices may be a mixture (i.e. a blend of surfactants, or an aqueous solution). For microemulsion systems, a phase is defined as a macroscopically optically homogeneous region, as opposed to the classical thermodynamic definitions which implies molecular or intimate mixing.

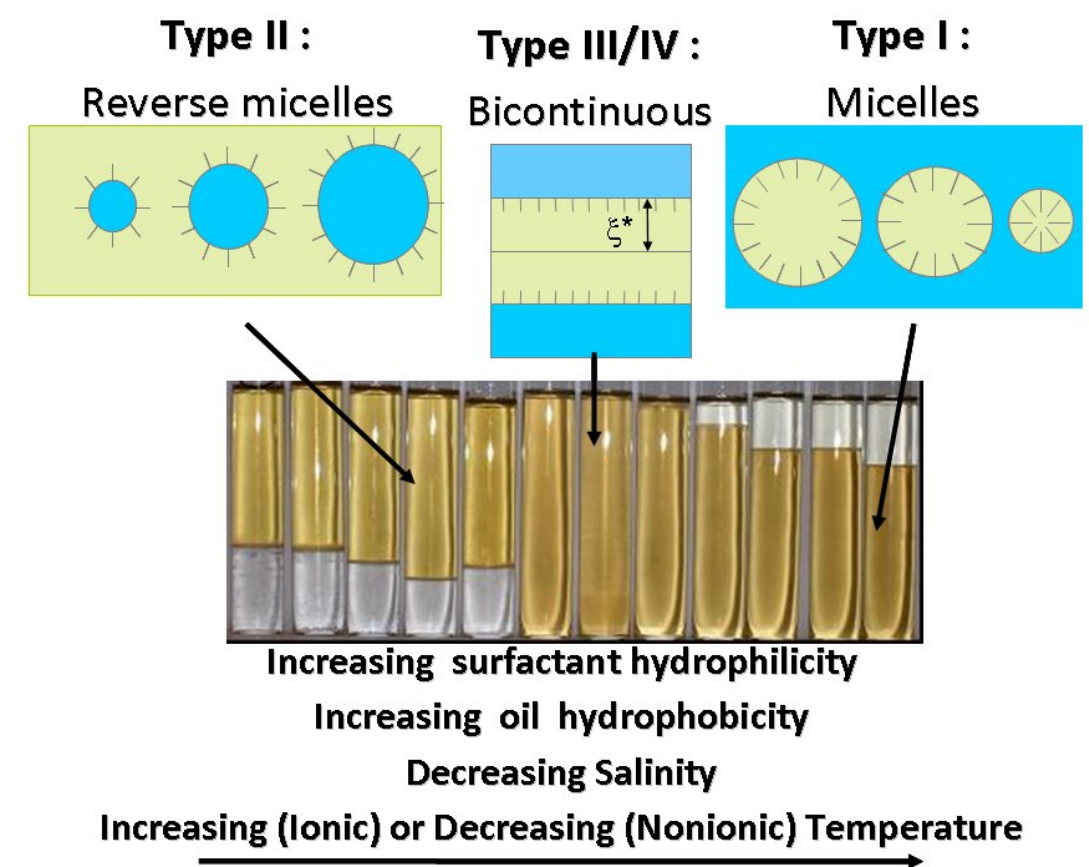

Figure 1-5: Typical microemulsion structures and dependence on formulation variables. The persistence length is marked by $\xi$ for bicontinuous microemulsions. 


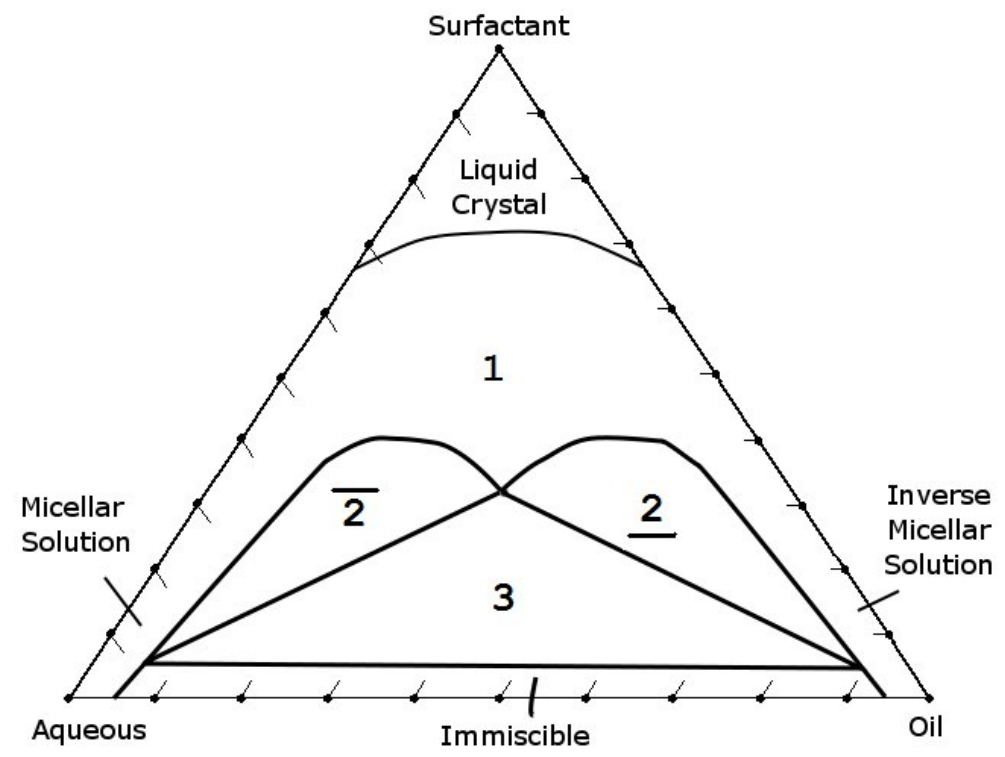

Figure 1-6: Sample pseudo-ternary diagram. Microemulsion regions are marked by number of phases. Bars indicate location of microemulsion for biphasic regions. The three phase region contains a middle microemulsion phase.

\subsubsection{The linker effect}

One of the tools that have been used to produce microemulsions with complex hydrophobic oils is the linker formulation approach ${ }^{60,61}$. According to this approach, the hydrophilic-lipophilic interaction can be balanced by mixtures of hydrophilic and lipophilic surfactants that form a series of mediating connections to enhance the solubilisation of one phase into the other ${ }^{34,62,63}$. This effect is extended through the use of surfactant-like components that co-adsorb near the interface, while remaining segregated into the oil (lipophilic linker) or aqueous (hydrophilic linker) phase ${ }^{64,65}$. The concept was first developed by Graciaa et al., who found that long-chain alcohols and ethoxylated fatty acids preferentially adsorbed near the surfactant tails, and increased oil solubilisation ${ }^{66,67}$. The linker approach is particularly useful when forming 
microemulsions with oils that are difficult to solubilize, essentially replacing a few higher energy interactions with a series of lower energy interactions. The linker effect is shown schematically in Figure 1-7.

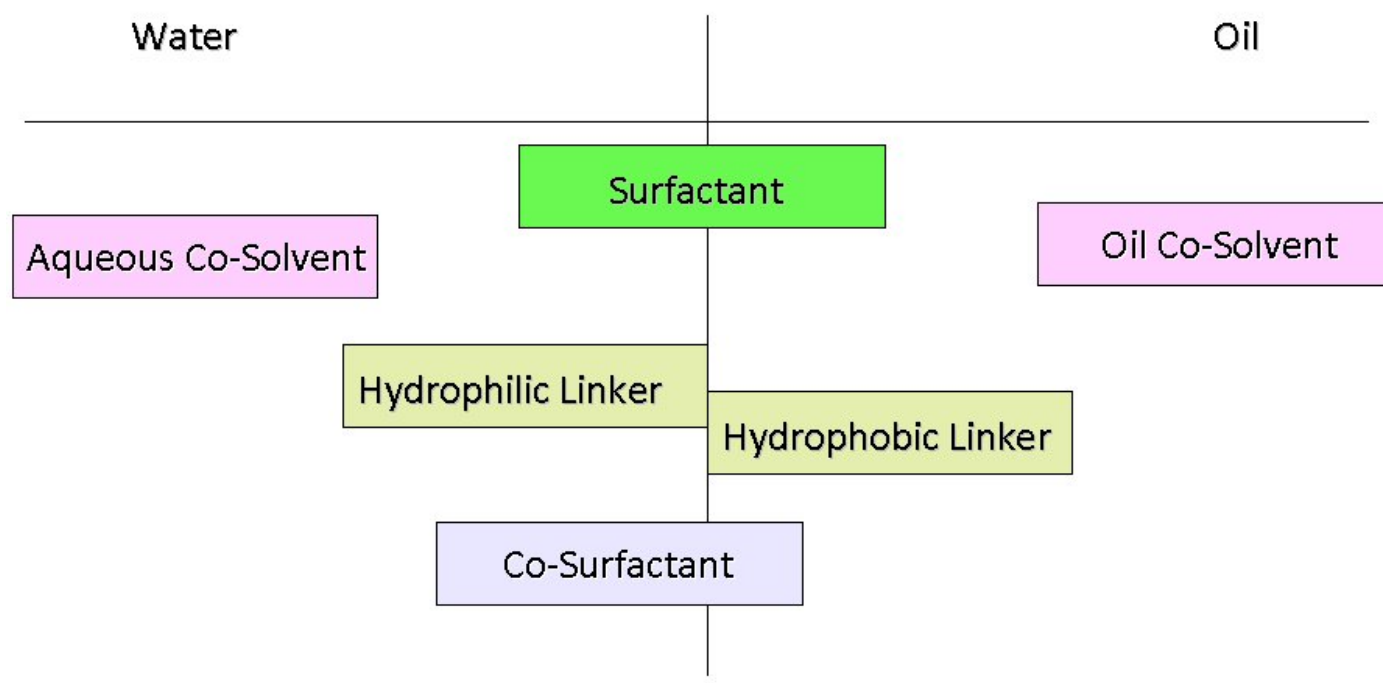

Figure 1-7: Schematic representation of the linker effect. The use of co-surfactants and linkers extends the interfacial ordering of the surfactant deeper into each phase. The location and overlap of boxes is intended to reflect the relative distribution of each species.

\subsection{MICROEMULSION CHARACTERIZATION}

Microemulsions are commonly characterized through several means. Microstructure can be distinguished through scattering techniques such as small angle neutron scattering (SANS), small angle x-ray scattering (SAXS) and for dilute droplets, dynamic light scattering (DLS). Select theoretical models for characterizing microemulsion structure and phase behaviour are presented below. 


\subsubsection{Persistence Length}

The persistence length of a bicontinuous microemulsion, $\xi$, is a measure of channel radius in the interpenetrating domains, as noted in Figure 1-5. The approach to calculating this characteristic length was originally developed by Scriven et al. ${ }^{68}$, Talmon et al. ${ }^{69}$ and finally simplified by De Gennes and Taupin ${ }^{51}$, and is based the geometric and surfactant surface concentration constraints in a bicontinuous microemulsion. The persistence length is given by:

1-6: $\xi=Z \times\left(\frac{\varphi_{\text {oil }} \varphi_{\text {aqueous }}}{C_{s} A_{s}}\right)$

Where $\varphi_{\text {oil }}$ and $\varphi_{\text {aqueous }}$ are the volume fractions of the oil and aqueous phase, respectively, $C_{s}$ is the surfactant concentration, and $\mathrm{A}_{\mathrm{s}}$ is the area per surfactant molecule. $\mathrm{Z}$ is a constant, estimated as $5.82^{69}, 6^{51}$ and $7.16^{70}$. In this work, we use the value $\mathrm{Z}=6$ from De Gennes' simplified model for consistency with other reports ${ }^{71,72}$. The effective area per molecule, $A_{s}$ can be estimated by solving the Gibbs Adsorption equation for surface concentration at constant $\mathrm{T}$, given by:

1-7: $\Gamma=\frac{1}{2.303 R T}\left(\frac{\partial \gamma}{\partial \log C}\right)_{T}$ and 
1-8: $A_{s}=\frac{10^{16}}{N \times \Gamma}$

Where $\Gamma$ is the surface concentration in $\mathrm{mol} / \mathrm{cm}^{2}, \mathrm{C}$ is the molecular concentration of the surfactant, $\mathrm{N}$ is Avogadro's number, and $\mathrm{A}_{\mathrm{s}}$ is given in $\AA^{2} /$ molecule. $^{53,73,74}$

\subsubsection{Percolation}

Conductance measurement is a technique for measuring the connectivity of the aqueous phase through a phenomenon known as percolation. Where the aqueous phase is completely continuous (dilute $\mathrm{o} / \mathrm{w}$ ), the conductance is expected to be high. Where the aqueous phase is dispersed discretely (dilute w/o), the conductance is expected to be near-zero. Percolation is modeled as a mechanism for mass or ion transfer between dispersed droplets, as shown in Figure 1-8. Conductance percolation refers to an increase in charge transfer as either aqueous concentration (increase in size/number of droplets) or temperature (higher energy) increases. Fusion and fission of nearby droplets is continuous and dynamic, thus even a system with discrete water droplets may conduct if it contains sufficient water. The concentration or temperature where aqueous phase connectivity is achieved is marked by a sharp increase in conductance. $^{54,75}$ 


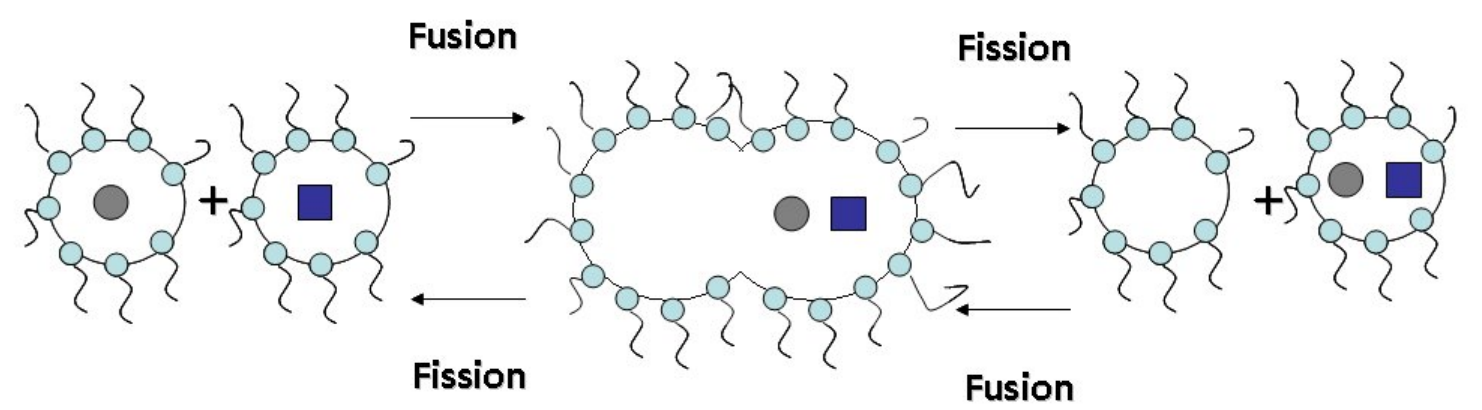

Figure 1-8: Mass/ion transfer between w/o droplets.

\subsubsection{The Winsor Ratio}

The Winsor Ratio is among the original parameterized models linking microemulsion phase behaviour to the physicochemical interactions at the interface. Winsor proposed that interaction between the water and surfactant $\left(\mathrm{A}_{\mathrm{SW}}\right)$ and between the oil and surfactant $\left(\mathrm{A}_{\mathrm{SO}}\right)$ would promote solubilization and a decrease in interfacial tension. On the other hand, interactions between water molecules $\left(\mathrm{A}_{\mathrm{WW}}\right)$, between surfactant molecules $\left(\mathrm{A}_{\mathrm{HH}}\right.$ for hydrophilic side interactions, $\mathrm{A}_{\mathrm{LL}}$ for lipophilic side interactions) and between oil molecules $\left(\mathrm{A}_{\mathrm{OO}}\right)$ would hinder miscibility

and promote phase separation ${ }^{25,32,48,52}$. The interaction scheme is shown in Figure 1-9. The Winsor Ratio, R, relates these interactions by:

1-9: $R=\frac{A_{S O}-A_{o o}-A_{L L}}{A_{S W}-A_{W W}-A_{H H}}$ 
Winsor noted that a value of $\mathrm{R}<1$ implies greater interaction between the surfactant and hydrophilic phase, supporting a tendency towards the formation of micelles and type I microemulsions. For values of $\mathrm{R}>1$, there is a tendency towards reverse micelles and type II microemulsions. For value or $\mathrm{R} \approx 1$, the curvature of the interface approaches 0 and the system is bicontinuous. This is known as the optimum formulation, as it also corresponds to maximum solubilization. ${ }^{32,52}$ However, while the $\mathrm{R}$ value provides a simple approach to evaluating microemulsion phase behaviour, measurement of the component interactions remains a barrier to widespread use as a formulation tool.

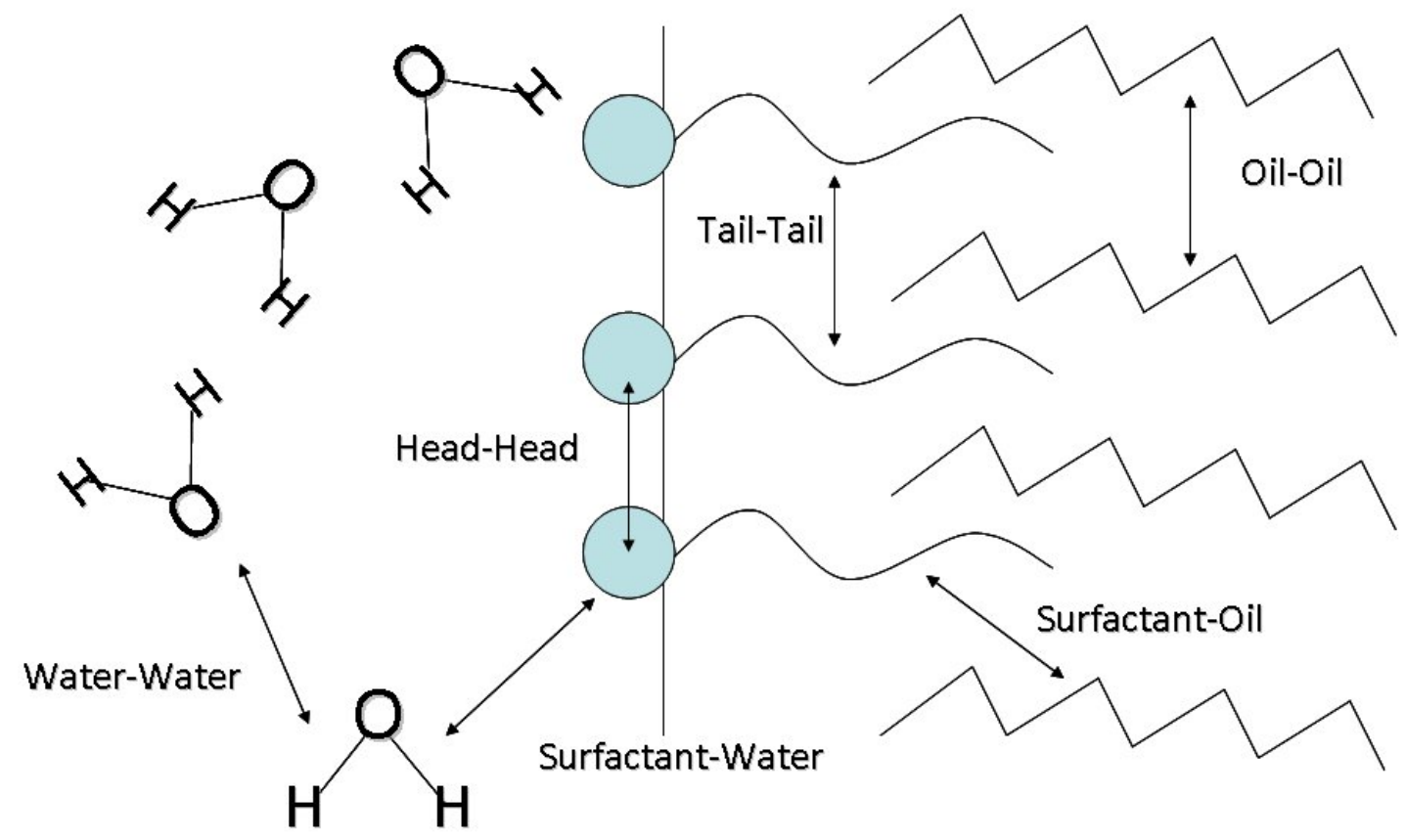

Figure 1-9: Schematic depiction of oil, water and surfactant interactions 


\subsubsection{Hydrophilic - Lipophilic balance (HLB) and Phase Inversion Temperature}

The hydrophilic - lipophilic balance (HLB) is the most commonly used characterization tool in surfactant literature and commercially, and was originally proposed by Griffin in $1949^{76-78}$. The HLB is given by:

1-10: $H L B=20 \times \frac{M_{h}}{M_{t}}$

Where $M_{h}$ is the molecular mass of the hydrophilic part of the molecule and $M_{t}$ is the total mass. Thus HLB values are reported on a scale of 0 to 20 . Oils for emulsification applications are assigned a "required" HLB value, which indicates a range of appropriate surfactants. The HLB approach provides a simple, arithmetical labeling system and is considered an industry standard 79,80 . The HLB of a mixture of surfactants is based on a linear average by weight ${ }^{52}$. However, the HLB does not account for formulation variables, and is thus limited to simple formulations with explicitly designed emulsification applications.

The phase inversion temperature is another empirical approach to characterization that simply measures the temperature at which a polyethoxylated nonionic surfactant partitions preferentially from the aqueous to oil phase, thus "inverting" the interface and causing a transition in microemulsion type. This temperature is considered equivalent to a Winsor Ratio of $\mathrm{R}=1^{52}$. 


\subsubsection{Hydrophilic-Lipophilic Difference (HLD)}

The hydrophilic-lipophilic-difference (HLD) is a semi-empirical equation for predicting and evaluating microemulsion phase behaviour for a given set of formulation conditions ${ }^{52}$. The value computed for a specific formulation reflects the curvature of the interface, so that an $\mathrm{O} / \mathrm{W}$ $\mu \mathrm{E}$ has an $\mathrm{HLD}<0$, while a $\mathrm{W} / \mathrm{O} \mu \mathrm{E}$ has an HLD $>0$. An HLD value of 0 corresponds to the optimal formulation, though in practice the range of values near zero that yield a bicontinuous microemulsion depends on the formulation. ${ }^{32,52,81-86}$. The HLD equations were constructed from simple empirical relationships ${ }^{87}$, relating phase behaviour to salinity, temperature, the nature of the oil, the nature of the surfactant, and the nature of a co-solvent. Though originally based on fitted parameters, recent efforts to characterize the natures of the oil and surfactants have led to a better understanding of the link between molecular structure and HLD contribution 71,88. Appendix A present examples of estimated characteristic values for surfactants and oils, respectively.

The HLD equation for ionic surfactants ${ }^{85}$ is given as:

$$
\text { 1-11: HLD }=\ln (S)-K * E A C N-f(A)-\alpha \Delta T+C_{c}
$$

For nonionic surfactants, the equation is ${ }^{87}$ :

$$
\text { 1-12: HLD }=b(S)-K * E A C N-\varphi(A)+c \Delta T+C_{c n}
$$

In this equation, $\mathrm{S}$ is the electrolyte concentration in the aqueous phase $(\mathrm{g} / 100 \mathrm{~mL})$. $\mathrm{EACN}$ is the equivalent alkane carbon number, which is a measure of the hydrophilic-lipophilic nature of the oil. For alkanes, this is simply the number of carbons in the chain. The co-surfactant 
functions $\mathrm{f}(\mathrm{A})$ and $\varphi(A)$ depend on the type and concentration of alcohol, or co-solvents. The terms $\mathrm{C}_{\mathrm{c}}$ and $\mathrm{C}_{\mathrm{cn}}$ represent characteristic curvatures for ionic or nonionic surfactants. $\Delta \mathrm{T}$ is the deviation from a reference of $298 \mathrm{~K}$. The terms $\mathrm{b}, \mathrm{K}, \alpha$ and $\mathrm{c}$ are empirical constants that depend on the system being studied, and are estimated by plotting the HLD variation with each respective term while holding the others constant for that system.

The HLD equation provides an "equation of state" for microemulsions that can be used to predict the formation of a desired morphology and to compare the hydrophilic-lipophilic nature of oils and surfactants. A limitation of the equation is that it was developed with experimental data generated with formulations that used oil/water ratios of 1 , and surfactant concentrations typically lower than $30 \%{ }^{84,86}$. Despite this limitation, the HLD provides the most consistent basis for quantifying and comparing unknown oils and surfactants.

\subsection{MOTIVATION, HYPOTHESES AND OBJECTIVES}

The primary motivation for this work is the development of biomaterials suitable for use in physiological fluid-contacting applications. Modern soft contact lenses, for example, are commonly made of silicone hydrogels - the silicone phase contributes the required mechanical properties and gas transport pathways, while the hydrogel phase provides the aqueous transport pathways. Applications in medical device coatings, microfluidic substrates in blood-contacting devices and drug delivery devices can have similar requirements. Thus, the overall goal of this project is to develop a simple method for formulating IPNs of hydrophobic and hydrophilic 
polymers using microemulsion morphology as a template. This process would eliminate the need for multiple curing and equilibration steps, while still allowing for a wide range of materials and nanostructures to be developed. The thermodynamics of polymer blends suggest that the critical obstacle in mixing polymers is the enthalpy of mixing, $\Delta \mathrm{H}_{\text {mix }}$. This is particularly a problem when the mixture includes silicone rubbers, which are largely immiscible with other polymers and are particularly immiscible with hydrophilic polymers ${ }^{3}$. The introduction of interfacial agents to reduce interfacial tension has met with general success in polymer blends ${ }^{4,5}$. Here we investigate applying the low interfacial tensions and domain sizes common to microemulsions to the problem of polymer miscibility. By introducing polymerizable components into the oil and aqueous phases of a microemulsion, we may essentially create a low viscosity, low interfacial tension, bicontinuous template with nanostructured morphologies and narrow domain size distributions analogous to those generated through conventional IPN synthesis and spinodal decomposition. Polymerization of this formulation would ideally result in permanent crosslinks that will arrest polymerization induced phase separation, ensuring nanoscale mixing at both the monomeric and polymeric stages. However, while hydrocarbon microemulsions have been well studied, research into the behaviour and characterization of silicone-based microemulsions is limited, and attempts at polymerization remain restricted by an inability to preserve the microemulsion structure through the disruptive polymerization process. Thus, the hypothesis driving this work is summarized as follows: 
IF THE INTERPENETRATING MORPHOLOGY OF A BICONTINUOUS

MICROEMULSION CONTAINING REACTIVE MONOMERS CAN BE

STABILIZED, THEN POLYMERIZATION OF BOTH PHASES MAY YIELD AN INTERPENETRATING POLYMER NETWORK.

A critical component of this hypothesis is the extension of the classical IPN definition to include interpenetrating networks on a new scale of entanglement. In conventional IPNs, spinodal decomposition leads to a scale of entanglement at the level of polymer chains, whereas microemulsion templated IPNs are expected to be entangled on the scale of groups of polymer chains. This hypothesis leads to a series of secondary hypotheses and objectives that must be addressed. The first part of the project focuses on contributing to an understanding of how silicone microemulsions can be formulated and controlled. By building a consistent foundation for understanding how a formulation will behave under the conditions required for polymerization, polymerizable microemulsions that retain their initial nanostructure may be more easily designed. The second part of the project focuses on developing a methodology for polymerizing reactive, bicontinuous microemulsions containing silicone oil, methacrylic acid and hydroxyethyl methacrylate

The major objectives are summarized below:

1. Formulate a bicontinuous microemulsion containing reactive monomers in aqueous solution and silicone oil

a. Characterize the contributions of each component to the phase behaviour

b. Evaluate the phase behaviour in order to confirm bicontinuity, and to investigate stability over a range of temperatures and $\mathrm{pH}$ 


\section{Produce and characterize a silicone hydrogel with a bicontinuous morphology from a bicontinuous microemulsion template}

\section{a. Develop a polymerization method}

\section{b. Compare non-reactive and polymerizable surfactants}

\subsection{THESIS OUTLINE}

This dissertation is divided into four chapters designed to address a series of hypotheses and objectives based on the primary hypothesis and objectives described above. Chapter 2 presents an investigation of silicone oils, silicone surfactants and silicone microemulsions according to the HLD framework, according to the first major objective. Chapter 3 continues the discussion of silicone microemulsion characterization with a focus on the specific impact of monomer solubility vs. solubilization as it relates to the formulation of reactive microemulsions. Chapter 4 addresses the second major objective by introducing the methodology for exploiting the microemulsions developed in earlier chapters as reactive templates for silicone hydrogels, while also exploring techniques to minimize disruption and preserve the microemulsion structure. Chapter 5 examines the link between microemulsion phase behaviour and polymer microstructure, showing that it is possible to generate a variety of structures by changing the microemulsion template. Finally, chapter 6 summarizes the findings of this thesis and offers suggestions for future work. 


\subsection{REFERENCES}

1. Kumar, A.; Gupta, R. K. Fundamentals of Polymers; McGraw-Hill: 1998.

2. Young, R. J.; Lovell, P. A. Introduction to Polymers; Chapman and Hall: London, 1991; pp 443.

3. Lucas, P.; Robin, J. -J. Advances in Polymer Science 2007, 1, 111-147.

4. Robeson, L. M. Polymer Blends, A Comprehensive Review; Carl Hanser Verlag Edu: Munich, 2007.

5. Shonaike, G. O.; Simon, G. P. Polymer Blends and Alloys; Marcel Dekker: New York, 1999.

6. Turner, J. S.; Cheng, Y. -L. Macromolecules 2003, 6, 1962-1966.

7. Giordano, N.; Cheng, J. -T. J. Phys. : Condens. Matter 2001, R271-R295.

8. Bhattacharya, S.; Datta, A.; Berg, J. M.; Gangopadhyay, S. J Microelectromech Syst 2005, 3, 590(8)-598.

9. Duffy, D. C.; McDonald, J. C.; Schueller, O. J. A.; Whitesides, G. M. Anal. Chem. 1998, 4974-4984.

10. Lipatov, Y. S.; Nesterov, A. E. Thermodynamics of Polymer Blends; Technomic: Lancaster, USA, 1997; Vol. 1, pp 450.

11. Huggins, M. L. J. Chem. Phys. 1941, 5, 440.

12. Flory, P. J. J. Chem. Phys. 1942, 1, 51-61.

13. Kiefer, J.; Hedrick, J.; Hilborn, J. 1999, 161-247.

14. Hagiopol, C. Copolymerization: Towards a systemic approach; Kluwer Academic: New York, 1999; Vol. 2.

15. Klempner, D.; Frisch, K. C. In Advances in Interpenetrating Polymer Networks. Technomic: Lancaster, PA, 1994; .

16. Sperling, L. H. Interpenetrating Polymer Networks and Related Materials; Plenum: New York, 1981; .

17. Sperling, L. H.; Mishra, V. Polym. Adv. Technol. 1996, 4, 197-208.

18. Park, J. W.; Kim, S. C. Phase Separation During Synthesis of Polyetherimide/Epoxy SemiIPNs. In IPNs Around the World; Kim, S. C., Sperling, L. H., Eds.; John Wiley: Chichester, England, 1997; pp 27.

19. Tran-Cong, Q.; Kawai, J.; Nishikawa, Y.; Jinnai, H. Physical Review E - Statistical Physics, Plasmas, Fluids, and Related Interdisciplinary Topics 1999, 2 B, R1150-R1153.

20. Binder, K.; Stauffer, D. Phys. Rev. Lett. 1974, 17, 1006-1009.

21. Chou, Y. C.; Lee, L. J. Polym. Eng. Sci. 1994, 16, 1239-1249.

22. Cahn, J. W. Acta Metallurgica 1961, 9, 795-801. 
23. Cahn, J. W. J. Chem. Phys. 1965, 1, 93-99.

24. Hoar, T. P.; Schulman, J. H. Nature 1943, 3847, 102-103.

25. Winsor, P. A. Solvent Properties of Amphiphilic Compounds; Butterworths: London, 1954; .

26. Winsor, P. A. Transactions of the Faraday Society 1948, 376-398.

27. Sjoeblom, E.; Friberg, S. Journal of Colloid and Interf 1978, 16.

28. Ekwall, P. Composition, properties and structures of liquid crystalline phases in systems of amphiphilic compounds. In Advances in Liquid Crystals; Brown, G. H., Ed.; Academic Press: New York, 1975; pp 1.

29. Gillberg, G.; Lehtinen, H.; Friberg, S. J. Colloid Interface Sci. 1970, 1, 40-53.

30. Friberg, S. E.; Aikens, P. A. A Phase Diagram Approach to Microemulsions. In Microemulsions: Properties and Applications; Fanum, M., Ed.; CRC Press: Boca Raton, Florida, 2009; pp 1.

31. Danielsson, I.; Lindman, B. Colloids and Surfaces 1981, 4, 391-392.

32. Salager, J.; Antón, R. E.; Sabatini, D. A.; Harwell, J. H.; Acosta, E. J.; Tolosa, L. I. J Surfact Deterg 2005, 1, 3-21.

33. Shinoda, K.; Lindman, B. Langmuir 1987, 2, 135-149.

34. Fanum, M. Microemulsions with Mixed Nonionic Surfactants. In Microemulsions: Properties and Applications; Fanum, M., Ed.; CRC Press: Boca Raton, Florida, 2009; pp 87.

35. Holmberg, K. Microemulsions in Biotechnology. In Industrial Application of Microemulsions; Solans, C., Kunieda, H., Eds.; Marcel Dekker: New York, 1997; pp 69.

36. Fredon, L.; Mallard, C. Ann. Dermatol. Venereol. 2007, 3 C2, 2S30-2S36.

37. Patravale, V. B.; Mandawgade, S. D. Int. J. Cosmetic Sci. 2008, 1, 19-33.

38. Lawrence, M. J.; Rees, G. D. Advanced Drug Delivery Reviews 2000, 1, 89-121.

39. Carrión, F. J. Boletin Intexter del Instituto de Investigacion Textil y de Cooperacion Industrial 2007, 131, 17-25.

40. Hou, A.; Chen, S. J. Dispersion Sci. Technol. 2010, 1, 102-107.

41. Parvinzadeh, M.; Hajiraissi, R. Journal of Surfactants and Detergents 2008, 4, 269-273.

42. Hashem, M.; Ibrahim, N. A.; El-Shafei, A.; Refaie, R.; Hauser, P. Carbohydr. Polym. 2009, 4, 690-703.

43. Zhang, D.; Jiang, X.; Yang, C. J Appl Polym Sci 2003, 13, 3587-3593.

44. Zhang, D.; Jiang, X.; Yang, C. J Appl Polym Sci 2005, 1, 347-352.

45. Xu, Y.; Liu, Y.; Zhao, N.; Yuan, C. Huagong Xuebao/Journal of Chemical Industry and Engineering (China) 2006, 6, 1464-1467.

46. Guerrero-Ramírez, L. G.; Nuño-Donlucas, S. M.; Cesteros, L. C.; Katime, I. Materials Chemistry and Physics 2008, 3, 1088-1092. 
47. Sharma, S. C.; Tsuchiya, K.; Sakai, K.; Sakai, H.; Abe, M.; Komura, S.; Sakamoto, K.; Miyahara, R. Langmuir 2008, 15, 7658-7662.

48. Gilchrist, C. A.; Rogers, J.; Steel, G.; Vaal, E. G.; Winsor, P. A. Journal of Colloid and Interface Science 1967, 3, 409-420.

49. Scriven, L. E. Nature 1976, 5573, 123-125.

50. Clausse, M.; Peyrelasse, J.; Heil, J.; Boned, C.; Lagourette, B. Nature 1981, 5834, 636-638.

51. Degennes, P. G.; Taupin, C. J. Phys. Chem. 1982, 13, 2294-2304.

52. Salager, J. Formulation Concepts for the Emulsion Maker. In Pharmaceutical Emulsions and Suspensions; Nielloud, F., Marti-Mestres, G., Eds.; MARCEL DEKKER, INC.: New York, 2000; pp 19.

53. Rosen, M. J. Surfactants and Interfacial Phenomena, 3rd edition; John Wiley and Sons: New York, 2004; .

54. Rakshit, A. K.; Moulik, S. P. Physiochemistry of W/O Microemulsions: Formation, Stability and Droplet Clustering. In Microemulsions: Properties and Applications; Fanum, M., Ed.; CRC Press: Boca Raton, Florida, 2009; pp 17.

55. Ruckenstein, E.; Chi, J. C. Journal of the Chemical Society, Faraday Transactions 2: Molecular and Chemical Physics 1975, 1690-1707.

56. Prince, L. M. Microemulsions: Theory and Practice; Academic Press: New York, 1977; .

57. Holmberg, K. Quarter century progress and new horizons in microemulsions. In Micelles, Microemulsions, and Monolayers Science and Technology; Shah, D. O., Ed.; Marcel Dekker: New York, 1998; pp 161-192.

58. Lindman, B.; Shinoda, K.; Olsson, U.; Anderson, D.; Karlström, G.; Wennerström, H. Colloids and Surfaces 1989, 1, 205-224.

59. Kumar, P.; Mittal, K. L. Handbook of microemulsion science and technology; Marcel Dekker: New York, 1999; .

60. Sabatini, D. A.; Acosta, E.; Harwell, J. H. Current Opinion in Colloids and Interface Science 2003, 4-5, 316-326.

61. Acosta, E.; Uchiyama, H.; Sabatini, D. A.; Harwell, J. H. Journal of Surfactants and Detergents 2002, 2, 151-157.

62. Silas, J. A.; Kaler, E. W.; Hill, R. M. Langmuir 2001, 15, 4534-4539.

63. Gee, R. P. Colloids and Surfaces A: Physicochemical and Engineering Aspects 1998, 1-3, 91-101.

64. Liu, J.; Huang, S.; Wu, S. Key Engineering Materials 2008, 497-500.

65. Liu, J.; Wu, S.; Huang, S.; Cong, P.; Mi, Y. Iranian Polymer Journal (English Edition) 2009, 2, 159-166.

66. Sharma, S. C.; Tsuchiya, K.; Sakai, K.; Sakai, H.; Abe, M.; Miyahara, R. Journal of Oleo Science 2008, 12, 669-673.

67. Hill, R. M. Current Opinion in Colloids and Interface Science 2002, 5-6, 255-261. 
68. Scriven, L. E. Nature 1976, 5573, 123-125.

69. Talmon, Y.; Prager, S. J. Chem. Phys. 1978, 7, 2984-2991.

70. Sottmann, T.; Strey, R.; Chen, S. -H. J. Chem. Phys. 1996, 15, 6483-6491.

71. Acosta, E. J. Colloids Surf. Physicochem. Eng. Aspects 2008, 1-3, 193-204.

72. Zemb, T. Comptes Rendus Chimie 2009, 1-2, 218-224.

73. Guest, D.; Auvray, L.; Langevin, D. Journal de physique.Lettres 1985, 22, 1055-1063.

74. Gentle, T. E.; Snow, S. A. Langmuir 1995, 8, 2905-2910.

75. Mehta, S. K.; Kaur, K.; Kaur, G.; Bhasin, K. K. Percolating Phenomenon in Microemulsions: Effect of External Entity. In Microemulsions: Properties and Applications; Fanum, M., Ed.; CRC Press: Boca Raton, Florida, 2009; pp 59.

76. Griffin, W. C. J.Soc. Cosmet.Chem. 1949, 311-326.

77. Griffin, W. C. Am Perfumer Essent Oil Rev 1955, 5, 26-29.

78. Griffin, W. C. J.Soc.Cosmet.Chem. 1954, 249-256.

79. Pasquali, R. C.; Sacco, N.; Bregni, C. Latin American Journal of Pharmacy 2009, 2, 313317.

80. Pasquali, R. C.; Taurozzi, M. P.; Bregni, C. Int. J. Pharm. 2008, 1-2, 44-51.

81. Anton, R. E.; Salager, J. L. J. Colloid Interface Sci. 1990, 1, 75-81.

82. Nardello, V.; Chailloux, N.; Poprawski, J.; Salager, J. -L.; Aubry, J. -M. Polym. Int. 2003, 4, 602-609.

83. Poprawski, J.; Catté, M.; Marquez, L.; Marti, M. -J.; Salager, J. -L.; Aubry, J. -M. Polym. Int. 2003, 4, 629-632.

84. Queste, S.; Salager, J. L.; Strey, R.; Aubry, J. M. J. Colloid Interface Sci. 2007, 1, 98-107.

85. Salager, J. L.; Morgan, J. C.; Schechter, R. S.; Wade, W. H.; Vasquez, E. Soc Pet Eng AIME $J$ 1979, 2, 107-115.

86. Bouton, F.; Durand, M.; Nardello-Rataj, V.; Serry, M.; Aubry, J. Colloids Surf. Physicochem. Eng. Aspects 2009, 1-3, 142-147.

87. Bourrel, M.; Salager, J. L.; Schechter, R. S.; Wade, W. H. J. Colloid Interface Sci. 1980, 2, 451-461.

88. Acosta, E. J.; Bhakta, A. S. Journal of Surfactants and Detergents 2009, 1, 7-19. 


\section{THE HYDROPHOBICITY OF SILICONE-BASED OILS AND SURFACTANTS AND THEIR USE IN REACTIVE MICROEMULSIONS}

The contents of this chapter have been published under the following citation:

Castellino, V., Cheng, Y.-L., Acosta, E. The hydrophobicity of silicone-based oils and surfactants and their use in reactive microemulsions (2011) Journal of Colloid and Interface Science, 353 (1), pp. 196-205

\subsection{INTRODUCTION AND BACKGROUND}

Microemulsions $(\mu \mathrm{E})$ are thermodynamically stable, nanostructured systems containing oil and water domains stabilized by surfactants. The nanostructures are typically seen as oil-in-water micelles $(\mathrm{O} / \mathrm{W}$, Winsor type I), water-in-oil reverse micelles (W/O, type II), or co-existing bicontinuous domains of water and oil. Theoretical understanding of microemulsion structure and phase behaviour has been a subject of interest since the $1940 \mathrm{~s}^{1-4}$. Recent interest in silicone oil based microemulsions has gravitated towards delivery systems for cosmetics and pharmaceuticals ${ }^{5-9}$, fabric treatments ${ }^{10-13}$, and nanoscale polymers ${ }^{14-18}$. The stability and phase behaviour of silicone oil based microemulsions with a range of surfactants have been previously characterized, with a focus on developing systems that emulate the solubilization and oil uptake behaviour of hydrocarbon microemulsions ${ }^{19-22}$. As a result, most studies of unmodified silicone oils have focused on the lowest viscosity silicone oil commercially available: 0.65 centistoke (cSt) hexamethyldisiloxane ${ }^{20-22}$. Solubilization of higher viscosity oils has required either altering the silicone chemistry to include hydrophilic side groups, or adding a solvent to the oil 
phase $^{11,18,23-28}$. The development of siloxane based surfactants has facilitated silicone oil solubilization ${ }^{29,30}$ and several groups have observed transparent microemulsion formation in silicone oil-water-silicone/siloxane surfactant systems, as well as ordered lamellar, hexagonal or cubic liquid crystal phases. The use of these surfactants and low molecular weight (90-200 Dalton) or modified silicone oils have resulted in the formation of true bicontinuous microemulsions over a limited range of temperatures and compositions ${ }^{18,20,28,31-34}$. However, research into the phase behaviour of microemulsions containing unmodified silicone oils and silicone-based surfactants, particularly higher molecular weight polydimethylsiloxane (PDMS), remains scarce. High molecular weight PDMS is desirable, among other reasons, to reduce the toxicity and volatility of the oil.

In order to characterize the phase behaviour of a surfactant-oil-water (SOW) system, we turn to the hydrophilic-lipophilic difference (HLD) framework. The HLD is a semi-empirical equation for predicting and evaluating microemulsion phase behaviour for a given set of formulation conditions ${ }^{35}$. The HLD value computed for a specific formulation reflects the curvature of the interface, so that an oil in water $(\mathrm{O} / \mathrm{W}) \mu \mathrm{E}$ has an $\mathrm{HLD}<0$, while a W/O $\mu \mathrm{E}$ has an HLD $>0$ and a bicontinuous $\mu \mathrm{E}$ has an HLD $\sim 0$. The HLD framework has previously been readily applied to hydrocarbon based microemulsions ${ }^{36-41}$. However, a mathematical approach to microemulsion formulation has yet to be extended to silicone oils and silicone surfactants. Here we investigate, for the first time, the application of the HLD concept towards characterizing the hydrophilic-lipophilic nature of silicone oils and silicone-based surfactants through the use of reference systems containing oils and surfactants with known equivalent alkane carbon number $(\mathrm{EACN})$ and characteristic curvature $\left(\mathrm{C}_{\mathrm{c}}\right)$. 
Microemulsion morphologies have already been exploited to synthesize polymer nanoparticles of controllable size. A review by Chow and $\mathrm{Gan}^{42}$ presents various methodologies for exploiting and controlling microemulsion properties for use as a nano-scale reaction template for polymers, and several groups have investigated polymerization of hydrocarbon monomers. However, while polymerization of silicone-containing microemulsions has been reported ${ }^{14,15}$, studies to date have generally focused on polymerization in the aqueous phase ${ }^{17,42-44}$. Of particular interest is the possibility of using bicontinuous nanostructures to synthesize bicontinuous network of polymers, similar to the morphology of interpenetrating polymer networks (IPNs). A combination of interest is incorporating a crosslinked silicone elastomer with a hydrogel using bicontinuous microemulsions of reactive systems as a polymerization template. One of the challenges in formulating these microemulsions is a limited ability to quantify and predict the behaviour of silicone oils and silicone surfactants in reactive microemulsions. In addition, the impact of high concentrations of reactive monomer on phase behaviour is not well understood. This chapter is guided by the following hypothesis:

\section{If silicone oils and surfactants are formulated into microemulsions, they can be characterized by the HLD framework.}

Thus there are two main objectives in this work: 1) To investigate the application of the HLD framework towards characterizing and quantifying the relative hydrophobicities of selected silicone oils and silicone surfactants, and to investigate the effect of reactive monomers on phase behaviour, 2) To investigate the phase behaviour of reactive microemulsions containing

silicone oil, silicone alkyl polyether and reactive monomers in aqueous solution with respect to composition, $\mathrm{pH}$ and temperature. 


\subsection{EXPERIMENTAL}

\subsubsection{Materials}

Methacrylic acid (MAA, 99\%), hydroxyethyl methacrylate (HEMA, 97\%), toluene (99.8\%), dodecane (99\%), sodium chloride (99.5\%), lithium hydroxide (98\%), cesium hydroxide solution (50 wt. \%), potassium hydroxide solution (50 wt. \%) and sodium dihexyl sulfosuccinate (SDHS) solution ( $80 \%$ in water) were obtained from Sigma Aldrich. $10 \mathrm{~N}$ sodium hydroxide was purchased from VWR Canada. Trimethylsiloxy-terminated silicone oil (non-reactive 0.65 and 3 cSt oil) was obtained from Gelest. The general formula for the silicone oils is $\mathrm{CH}_{3}\left[\mathrm{Si}\left(\mathrm{CH}_{3}\right)_{2} \mathrm{O}\right]_{\mathrm{N}} \mathrm{Si}\left(\mathrm{CH}_{3}\right)_{3}$, where $\mathrm{N}=1$ for the $0.65 \mathrm{cSt}$ oil and $\mathrm{N}=4$ to 7 for the $3 \mathrm{cSt}$ silicone oil $^{45}$. Biosoft N1-5 (C11.5E5, 98\%) was donated by Stepan Canada. Silicone alkyl polyether surfactants (Silube J208) were donated by Siltech Corporation. The J208 Silube series consists of lauryl polyethylene glycol (PEG-8) dimethicones. Properties of the surfactant line are presented in Table 2-1 ${ }^{46}$, and the general PEG-dimethicone structure is shown in Figure 2-1.

Table 2-1: Summary of surfactant properties ${ }^{46}$

\begin{tabular}{cccc}
\hline Surfactant & $\begin{array}{c}\text { Product } \\
\text { Designation }\end{array}$ & $\begin{array}{c}\text { Molecular Weight } \\
(\mathbf{g} / \mathbf{m o l})\end{array}$ & $\begin{array}{c}\text { Hydrophilic-Lipophilic } \\
\text { Balance (HLB) }\end{array}$ \\
\hline A & Silube J208-812 & 4500 & 3.2 \\
B & Silube J208-612 & 5000 & 5.6 \\
Y & Silube J208-412 & 5500 & 7.8 \\
Z & Silube J208-212 & 6000 & 9.6 \\
\hline
\end{tabular}




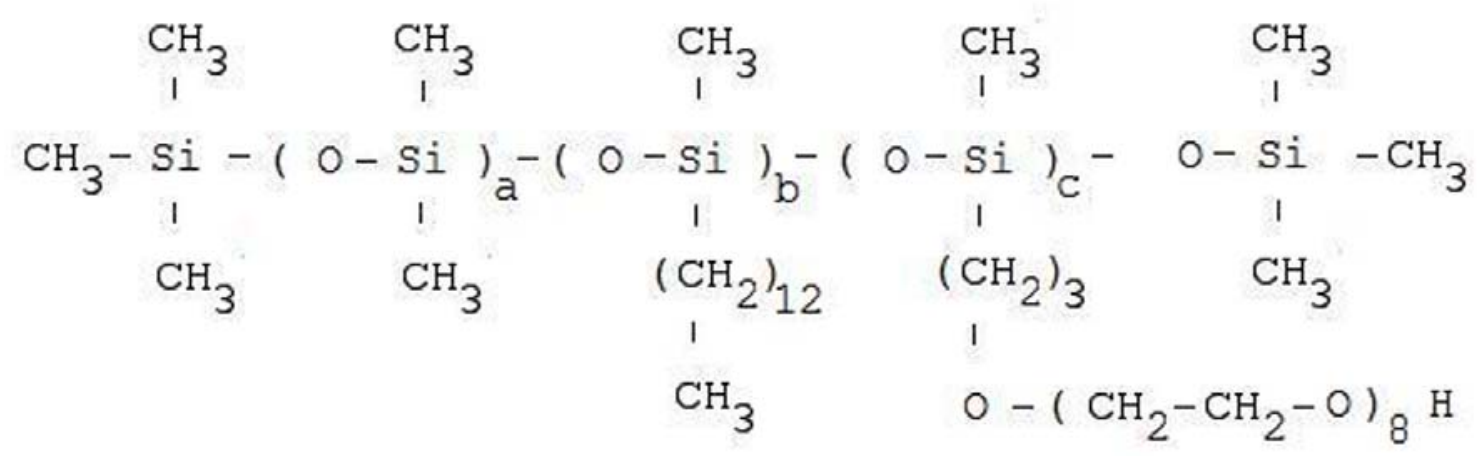

Figure 2-1: General structure of silicone alkyl polyether surfactant (PEG-8 Dimethicone). The hydrophilicity is determined by the ratio of polyether to silicone and alkyl groups. ${ }^{46}$

\subsubsection{Determination of silicone oil EACN}

The HLD equation for ionic surfactants is given as:

2-1: HLD $=\ln (S)-K * E A C N-f(A)-\alpha \Delta T+C_{c}$

For nonionic surfactants, the equation is:

2-2: HLD $=b(S)-K * E A C N-\varphi(A)+c \Delta T+C_{c n}$

In this equation, $\mathrm{S}$ is the electrolyte concentration in the aqueous phase $(\mathrm{g} / 100 \mathrm{~mL})$. EACN is the equivalent alkane carbon number, and is a measure of the hydrophilic-lipophilic nature of the oil, with polar oils having lower values. For alkanes, this is simply the number of carbons in the chain. The functions $\mathrm{f}(\mathrm{A})$ and $\varphi(A)$ depend on the type and concentration of alcohol, or cosolvents. The terms $\mathrm{C}_{\mathrm{c}}$ and $\mathrm{C}_{\mathrm{cn}}$ represent characteristic curvatures for ionic or nonionic surfactants. $\Delta \mathrm{T}$ is the deviation from a reference of $298 \mathrm{~K}$. The terms $\mathrm{b}, \mathrm{K}, \alpha$ and c are 
empirical constants that depend on the system being studied. The value of $b$ is typically 0.13 for sodium salts. The value of $\mathrm{K}$ can range from 0.1 to 0.2 , but is typically 0.16 for the nonionic reference systems used in the current work. Similarly the value of $\mathrm{c}$ for the nonionic reference system is typically 0.06 and the value of $\alpha$ for the ionic reference system is $0.01{ }^{47,48}$. The value for $\mathrm{K}$ in the ionic reference system was obtained by fitting 2-1 to a known oil, toluene, yielding $\mathrm{K}=0.145$.

It has also been found that both the EACN of an oil mixture and the characteristic curvature of a surfactant mixture can be expressed by simple linear mixing:

\section{2-3: $\operatorname{EACN}_{\text {mixture }}=\sum X_{i} * E A C N_{i}$}

and

\section{2-4: $\mathbf{C}_{\mathrm{c}}=\sum \mathbf{Y}_{\mathbf{j}} * \mathbf{C}_{\mathrm{cj}}$}

Where $\mathrm{X}_{\mathrm{i}}$ is the volume fraction of oil " $\mathrm{i}$ " in the oil phase, and $\mathrm{Y}_{\mathrm{j}}$ is the mole fraction of surfactant " $\mathrm{j}$ " in the surfactant blend ${ }^{37,49,50}$. As previously described, the shift from negative to zero to positive HLD is marked by a transition from Type I $\rightarrow$ Type III $\rightarrow$ Type II. By eliminating certain components in the formulation or holding them constant, the case for HLD = 0 can be exploited to establish simple linear relationships between variables. It is important to note, however, that the region of existence for bicontinuous microemulsions is not limited to a value of $H L D=0$, but rather includes a range of near-zero HLD values. This is most easily observed in a "fish" diagram plotting temperature, salinity, or other formulation variables against surfactant concentration. An example fish diagram can be found in Appendix B. In a 
typical diagram, the range of temperatures at which a three-phase region exists is a function of surfactant concentration. As the HLD value is linearly related to temperature, each temperature point corresponds to a different value of the HLD, indicating that a range of HLD values is also required to characterize the three phase region.

The EACNs of 0.65 and $3 \mathrm{cSt}$ silicone oils were determined in a reference system containing SDHS $\left(C_{c}=-0.92{ }^{50}\right)$ and toluene. The test oils were diluted with toluene in volume fractions from 0 to $100 \%$ in $10 \%$ increments, and then mixed in a $1: 1$ ratio with $0.1 \mathrm{M}$ SDHS in aqueous solution. $\mathrm{NaCl}$ was added in $0.25 \mathrm{~g} / 100 \mathrm{~mL}$ (aqueous) increments. Samples were vortexed to promote shearing of droplets, and allowed to equilibrate for 24 hours at $\mathrm{T}=20^{\circ} \mathrm{C}$. The salinity required for a transition from Type I $\rightarrow$ III (lower boundary) and from Type III $\rightarrow$ II (upper boundary) was recorded, marking the range of existence for the three phase region. The optimal salinity (salinity required to reach $\mathrm{HLD}=0$ ), $S *$ was estimated as the average between the upper and lower boundary. The EACN for each oil mixture was calculated from the optimal salinity and the $\mathrm{C}_{\mathrm{c}}$ for SDHS using equation 2-1 for HLD $=0$. The EACN of the silicone oil was then calculated from the mixing rule described by equation 2-3.

\subsubsection{Determining the characteristic curvature of silicone surfactants}

With the EACNs for the 0.65 and $3 \mathrm{cSt}$ silicone oils established, the characteristic curvature, $\mathrm{C}_{\mathrm{cn}}$, for the nonionic silicone-based surfactants was determined in a reference system containing

$0.65 \mathrm{cSt}$ oil and $\mathrm{C} 11.5 \mathrm{E} 5$ at $25^{\circ} \mathrm{C}$. The $\mathrm{C}_{\mathrm{cn}}$ for $\mathrm{C} 11.5 \mathrm{E} 5$ was previously calculated to be $0.63{ }^{51}$. 
Silicone-based surfactants were diluted with C11.5E5 in volume fractions from 0 to $100 \%$ in $10 \%$ increments. Formulations containing $47.5 \%$ water, $47.5 \% 0.65 \mathrm{cSt}$ silicone oil and 5\% surfactant were prepared for each surfactant mixture. Samples were vortexed and allowed to equilibrate. The characteristic curvature of the surfactant mixture was calculated by combining equations 2-2 and 2-4 for formulations at the reference temperature with no salt or alcohol contribution. For a type III microemulsion $(\mathrm{HLD}=0)$ under these conditions, the equation simplifies to:

2-5: $\sum \boldsymbol{Y}_{j} * C_{C N j}=K * E A C N$

Since the values for $\mathrm{K}, \mathrm{EACN}$ and the $\mathrm{C}_{\mathrm{CN}}$ of $\mathrm{C} 11.5 \mathrm{E} 5$ are known, the $\mathrm{C}_{\mathrm{CN}}$ for the remaining surfactant can be calculated.

\subsubsection{Aqueous solution containing acrylic monomers}

The aqueous phase was prepared using deionized water, HEMA and MAA at a volumetric ratio of 4:3:2 (9.4:1:1 molar ratio). HEMA and water are miscible at room temperature. The solubility of MAA in water is $89 \mathrm{~g} / \mathrm{L}$ at $25^{\circ} \mathrm{C}$, which increases with pH and the presence of HEMA. HEMA content facilitates the co-dissolution of HEMA and MAA. This solution had a $\mathrm{pH}$ of 2.5 , below the pKa of MAA (pKa $=4.58)$. In order to investigate phase behaviour above the $\mathrm{pKa}$ of MAA, a pH 6 solution was prepared by the addition of sodium hydroxide, resulting in of $27.09 \mathrm{~g} / 100 \mathrm{~mL}$ (aqueous) sodium methacrylate. Previous studies have found that the ionic strength in the empirical HLD equation is largely dependent on the cation, in this case sodium 
52. An estimate for effective ionic strength is therefore calculated based on the molarity of sodium, so that salinity $(\mathrm{S})=14.54 \mathrm{~g} / 100 \mathrm{~mL}$ (aqueous, $\mathrm{NaCl}$ equivalent).

\subsubsection{Microemulsion preparation and phase behaviour scans}

Mixtures of aqueous solution, trimethylsiloxy terminated silicone oil and surfactant blend were combined in appropriate volumes to populate a ternary phase diagram. The ternary diagrams are initially populated by $662 \mathrm{~mL}$ samples with compositional changes in $10 \%$ increments by volume (i.e. $40 \%$ aqueous, $40 \%$ oil, $20 \%$ surfactant by volume). When adjacent compositions showed a change in phase behaviour, additional samples were added in $2 \%$ increments in order to resolve the phase boundary. Mixtures were vortexed, allowed to equilibrate overnight, then characterized by the number of liquid phases visible, type of Winsor system observed (if any), turbidity, and the stability of the aqueous phase. Systems were deemed stable if the aqueous phases formed molecular solutions with no separation or precipitation of monomer or solid fragments. Systems were deemed unstable if separation or precipitation of components occurred within the aqueous phase, which is visibly different than the turbidity or cloudiness observed in the separation between aqueous and oil phases. Each formulation was also characterized by the critical surfactant concentration, $C^{*}$, which is the minimum amount of surfactant required to achieve a type IV system.

To determine the effect of temperature on phase behaviour, samples were placed in a water bath with a Thermo Electron Corporation Haake DC 30 circulator. Samples were allowed to 
equilibrate for 8 hours or longer, until there were no visible changes in state or structure. As heating of the microemulsions sometimes resulted in irreversible structural changes, new samples were used for each temperature. Phase transitions were initially identified by observation, and confirmed by conductance measurements on formulations that formed microemulsions.

\subsubsection{Microemulsion characterization: conductance, microscopy and viscosity}

Conductance was measured at $25^{\circ} \mathrm{C}$ using a VWR/Traceable portable conductivity meter with a glass probe (cell constant of 1) supplied by Microelectrodes, Incorporated. In order to examine the change in conductance with respect to composition in the ternary phase diagrams, $5 \mathrm{~mL}$ samples containing a 50/50 mixture by volume ( $7.3: 1$ by mol) of $3 \mathrm{cSt}$ silicone oil and surfactant blend were prepared, and were diluted incrementally with aqueous solution until phase separation occurred. Conductance, normalized to the aqueous phase, is reported for homogeneous samples only. The formulation was allowed to equilibrate for 8 hours before conductance measurement at each dilution step. Conductance is measured by inserting the platinum probes to the middle of the sample. The conductivity of the aqueous solution was also measured as a reference at $25^{\circ} \mathrm{C}$.

For single and multiphase samples in phase behaviour experiments and experiments investigating the effect of MAA and HEMA as non-aqueous solvents, the microemulsion phase 
was first identified by visual inspection, and was confirmed by comparing the relative volumes and conductance of each phase.

For homogeneous microemulsions formulated with $3 \mathrm{cSt}$ PDMS, the presence of liquid crystals was evaluated by using cross-polarizers in the following cases: Samples with greater than $70 \%$ surfactant by volume, samples along the aqueous-surfactant axis of the ternary phase diagram, and samples along the dilution line used to measure conductivity. Birefringence was assessed by depositing $500 \mu \mathrm{L}$ of sample onto glass microscope slides with glass cover slips, and examining the samples under the cross-polarizing filter.

Viscosity was measured at $25^{\circ} \mathrm{C}$ using a Gilmont GV-2200 falling ball viscometer and a stainless steel ball for homogeneous samples along the dilution line used to measure conductivity. Samples were loaded into the viscometer, and the time required for a stainless steel ball to drop vertically through the sample over a fixed distance was measured. Sample viscosity is calculated from the measured velocity. 


\subsection{RESULTS}

\subsubsection{EACN of Silicone Oils}

Results for the salinity scan on formulations containing mixtures of silicone oils with the test oil, toluene, are shown in Figure 2-2. The EACN of the oil mixture is derived from the average value for $\ln \mathrm{S}^{*}$ according to equation 2-1 and the EACN of the silicone oil is then calculated from the mixing relationship described in equation 2-3. The linear mixing relationship is validated in Figure 2-2, which contains plots of $\ln \left(\mathrm{S}^{*}\right)$ vs. toluene volume fraction for both the $0.65 \mathrm{cSt}$ and $3 \mathrm{cSt}$ silicone oils.
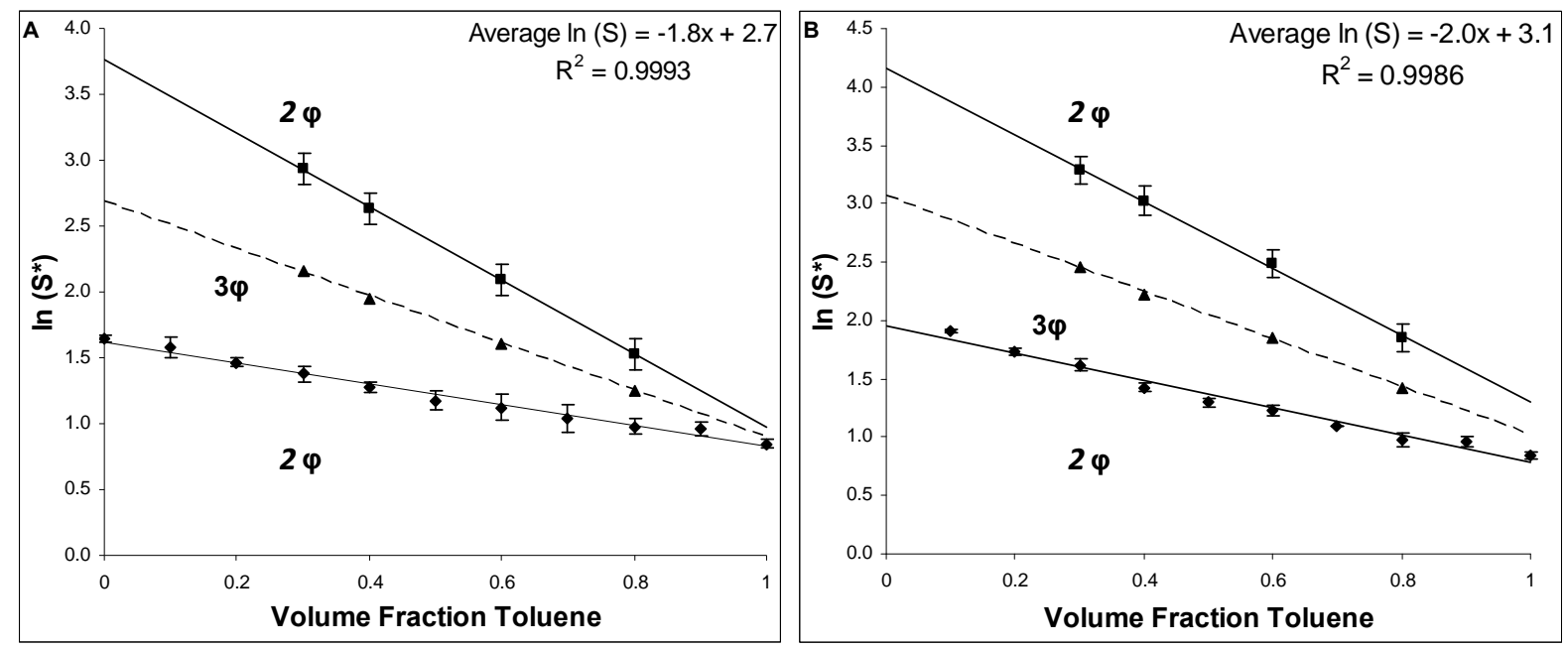

Figure 2-2: Salinity range required to form a three phase $\mu \mathrm{E}$ for mixtures of $0.65 \mathrm{cSt}$ silicone oil (A) or $3 \mathrm{cSt}$ silicone oil (B) with toluene as a function of toluene volume fraction in the oil blend. Dashed line represents the average value of $\ln S^{*} . T=20^{\circ} \mathrm{C}$. 
Since formulations at higher silicone oil content and higher salinity resulted in precipitation of the SDHS, we must extrapolate the range of salinities, and the optimal salinity $\mathrm{S}^{*}$, from the linear mixing relationship. The EACN for $0.65 \mathrm{cSt}$ silicone oil is estimated to be $12.3 \pm 0.7$, and the EACN for $3 \mathrm{cSt}$ silicone oil is estimated to be $15.0 \pm 0.7$.

\subsubsection{Comparison with Dodecane}

The calculated EACN of $12.3 \pm 0.7$ for $0.65 \mathrm{cSt}$ silicone oil implies that the oil behaves like $\mathrm{n}$ dodecane in the reference system studied. By definition, the alkane carbon number for $n-$ dodecane is 12 . In order to validate this comparison, the experiment was repeated with dodecane replacing 0.65 silicone oil, treating dodecane as oil with unknown EACN. The result is shown in Figure 2-3.

The salinity scan shows that optimal salinity, $\mathrm{S}^{*}$, for dodecane in the ionic reference system is similar to that of $0.65 \mathrm{cSt}$ silicone oil. Using the same values of $\mathrm{K}$ and $\alpha$ as for the silicone oils, this yields an EACN of 12 for dodecane and confirms an EACN of 1 for toluene. This validates both the assertion that $0.65 \mathrm{cSt}$ silicone oil behaves like dodecane, and the selection of parameters for the ionic reference system. 


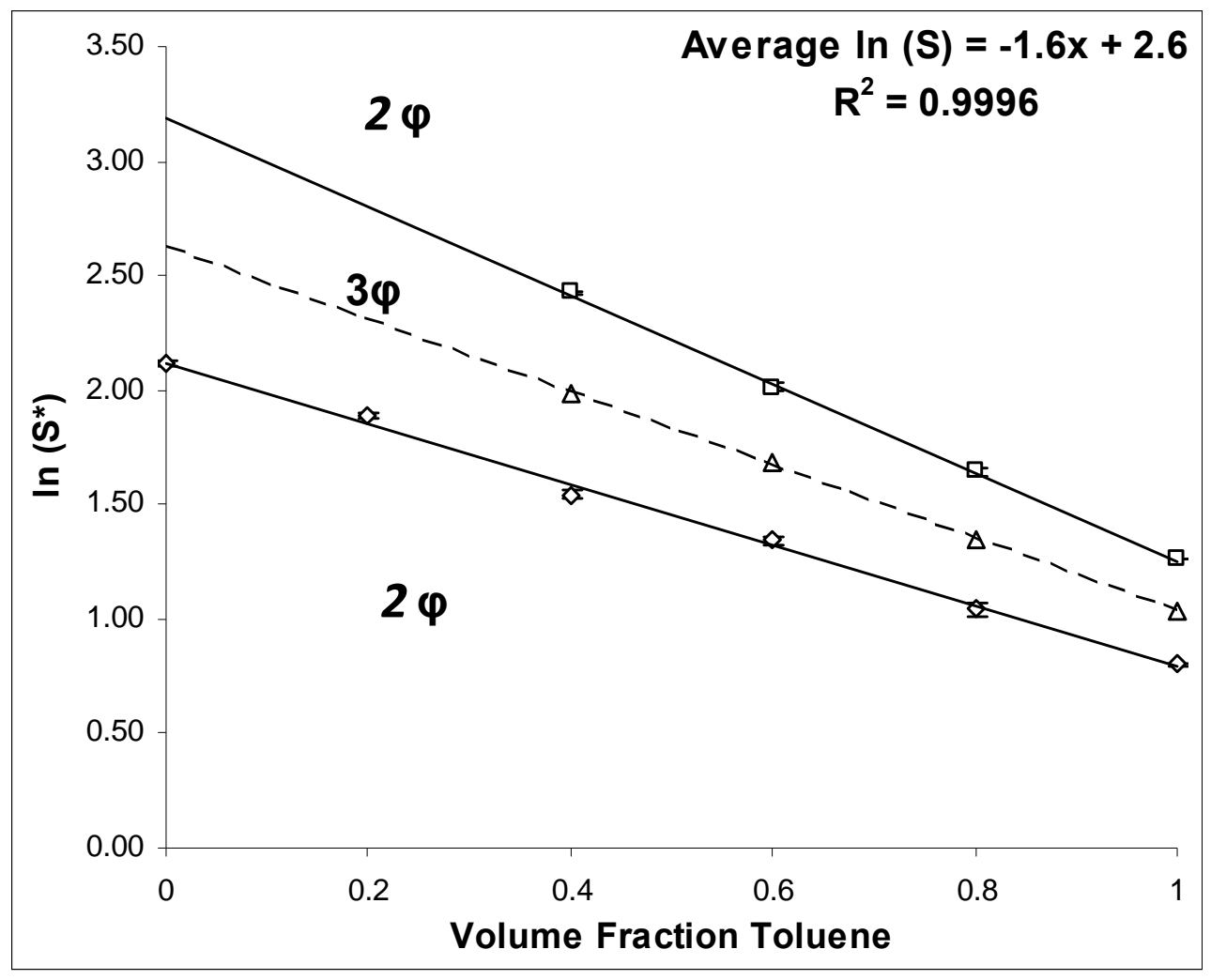

Figure 2-3: Salinity range required to form a three phase $\mu \mathrm{E}$ for mixtures of dodecane with toluene as a function of toluene volume fraction in the oil blend. Dashed line represents the average value of $\ln \mathrm{S}^{*} . \mathrm{T}=20^{\circ} \mathrm{C}$.

\subsubsection{Characteristic Curvature of Silicone Alkyl Polyether Surfactants}

Results for the phase behaviour scans on formulations containing $0.65 \mathrm{cSt}$ silicone oil, water, and a blend of C11.5E5 and silicone surfactant are summarized in Table 2-2. The calculated $\mathrm{C}_{\mathrm{CN}}$ values are based on the observed phase transitions and equation 2-5. 
Table 2-2: Calculated $\mathrm{C}_{\mathrm{CN}}$ values for the $\mathbf{J} 208$ series of silicone alkyl polyether surfactants in systems containing $0.65 \mathrm{cSt}$ silicone oil and deionized water.

\begin{tabular}{ccc} 
Test Surfactant & $\begin{array}{c}\text { Mole Fraction C11.5E5 at Phase } \\
\text { Transition }\end{array}$ & $\begin{array}{c}\text { Estimated } \\
\text { J208 C CN }_{\mathbf{C N}}\end{array}$ \\
\hline A & 0.91 & $18.9 \pm 1.1$ \\
B & 0.88 & $14.6 \pm 0.6$ \\
Y & 0.76 & $7.3 \pm 0.1$ \\
Z & 0.42 & 3.4 \\
\hline
\end{tabular}

\subsubsection{HLD Equation for Silicone-based Microemulsions and the Effect of HEMA/MAA}

A combination of surfactants A and B was selected for detailed phase behaviour investigation.

The selection of surfactants was based on preliminary phase behaviour studies that showed that the addition of the reactive monomers, HEMA and MAA, in most cases resulted in oil-in-water (type I) microemulsions, thus requiring the use of more hydrophobic silicone surfactants (surfactants A and B). Table 2-3 presents a sample calculation of HLD values using equation 2-2 for formulations containing 0.65 or $3 \mathrm{cSt}$ silicone oil, a blend of silicone alkyl polyether surfactants $\mathrm{A}$ and $\mathrm{B}$, and water with salinities of 0 (representing $\mathrm{pH} 2.5$ condition) and 14.54 $\mathrm{g} / 100 \mathrm{~mL}$ (to simulate equivalent ionic strength at $\mathrm{pH}$ 6). The positive sign of the HLD values in Table 2-3, in both cases, indicates that formulations with a mixture of surfactants A and B in water (no monomers) produce reverse micelles. 
Table 2-3: Calculated HLD values for formulations containing 0.65 or 3 cSt silicone oil, silicone alkyl polyether surfactant, and water with equivalent salinities of 0 and 14.54 $\mathrm{g} / 100 \mathrm{~mL}$. HLD values are calculated for a blend of surfactants $A$ and $B$ at $25^{\circ} \mathrm{C}$.

\begin{tabular}{ccccc}
$\begin{array}{c}\text { Volume Fraction of } \\
\text { Surfactant B in } \\
\text { Surfactant Blend }\end{array}$ & $\mathbf{0 . 6 5} \mathbf{c S t}$ HLD & $\mathbf{0 . 6 5}$ cSt HLD & $\mathbf{3}$ cSt HLD & $\mathbf{3}$ cSt HLD \\
\hline 0 & 17.0 & 18.9 & 16.5 & 18.4 \\
$(\mathbf{S}=\mathbf{0})$ & $(\mathbf{S}=\mathbf{1 4 . 5 4})$ & $\mathbf{( S = 0 )}$ & $\mathbf{1 4 . 5 4 )}$ \\
\hline 0.2 & 16.1 & 18.0 & 15.7 & 17.6 \\
0.4 & 15.3 & 17.1 & 14.8 & 16.7 \\
0.6 & 14.4 & 16.3 & 13.9 & 15.8 \\
0.8 & 13.5 & 15.4 & 13.1 & 15.0 \\
1 & 12.7 & 14.5 & 12.2 & 14.1 \\
\hline
\end{tabular}

According to Table 2-3, a more hydrophilic surfactant (e.g. a hydrophilic linker-like compound) should be used to bring the HLD values closer to zero, since the calculated HLD values are positive.

In this case, however, we compensate the hydrophobicity of the surfactant mixture with the mixture of HEMA and MAA as organic co-solvents that modify the hydrophilic environment of the aqueous phase. This approach has been used before in producing microemulsions with fluorocarbon-based oils ${ }^{53}$, and in producing microemulsion templated latex ${ }^{54}$. Unfortunately, the effect of this co-solvent mixture in the HLD, as reflected by $\varphi(A)$ in equation $2-2$, has not been defined in the literature.

To explore the use of mixtures of HEMA and MAA (3:2 volume ratio, 1:1 molar ratio) as aqueous co-solvents, the phase behaviour of $0.65 \mathrm{cSt}$ PDMS microemulsions was evaluated as a 
function of the volume fractions of HEMA/MAA in the aqueous phase containing a 50/50 volume ratio (1.1: molar ratio) of surfactant $\mathrm{A} / \mathrm{B}$ mixture. This phase behaviour is presented in Figure 2-4. According to this figure, increasing the HEMA and MAA content induced a transition from a type II (HLD $>0$ ) to a type III (HLD $~ 0)$ microemulsion. Conductance measurements also confirm a shift from oil-continuous to bicontinuous microemulsion. This observation corroborates that the addition of HEMA and MAA in aqueous solution produces a significant negative shift in HLD, allowing the formulation to approach bicontinuity.

Figure 2-5 shows the effect of surfactant $\mathrm{A} / \mathrm{B}$ composition on the phase behaviour and relative conductivity of the (water-MAA-HEMA) - (Surfactant A+B) - $(0.65 \mathrm{cSt})$ microemulsion. Surfactant mixtures that have more surfactant A (more hydrophobic) have slightly lower conductivities than microemulsions containing more surfactant $\mathrm{B}$. These observations are consistent with the difference in HLDs in Table 2-3 in that surfactant B, being more hydrophilic than surfactant A should produce slightly more hydrophilic formulations (i.e. higher relative conductivity in this case. 

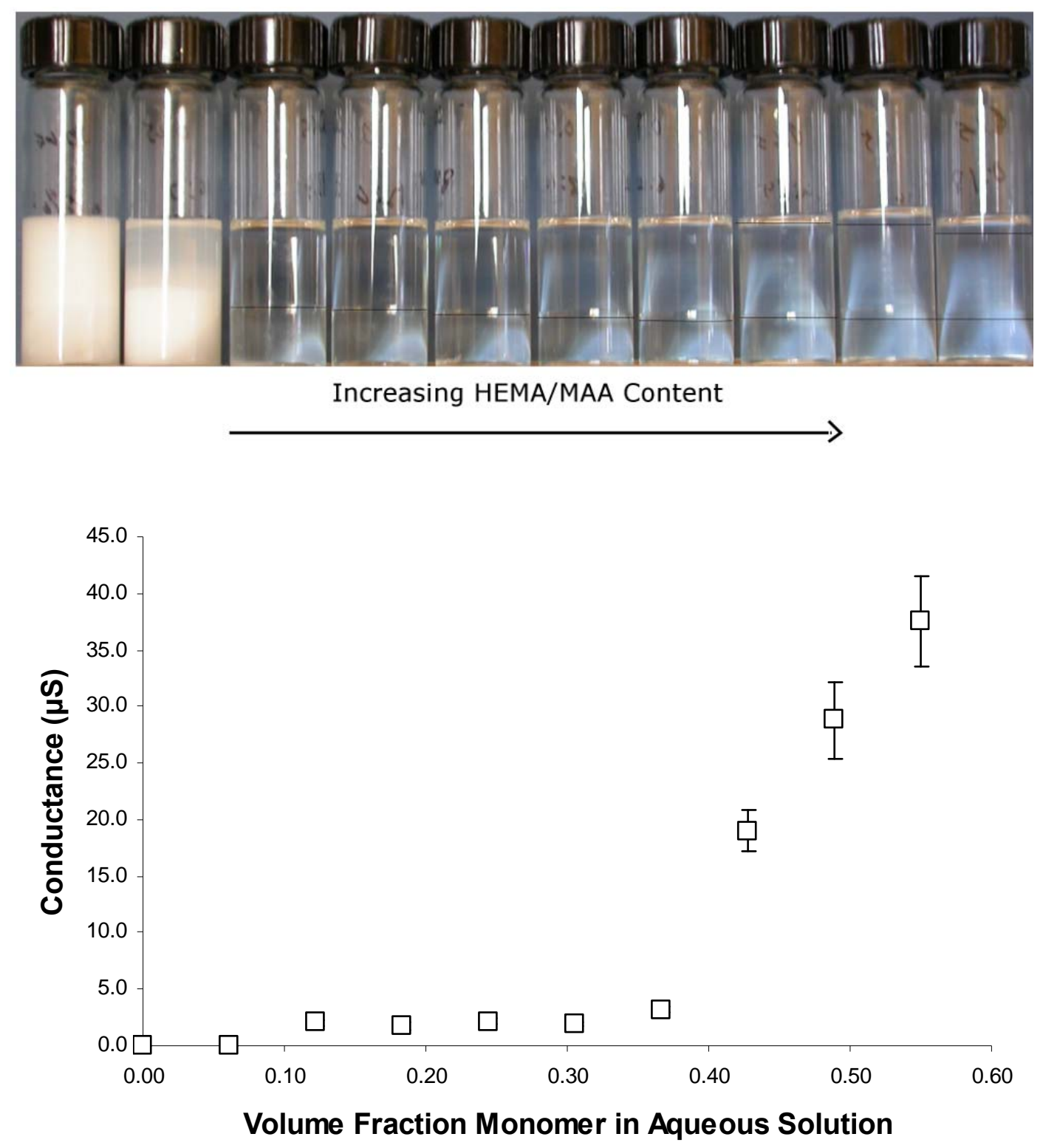

Figure 2-4: Effect of acrylic monomers on phase behaviour. A) Phase scan of formulations containing equal parts oil and aqueous phase and a 50/50 blend of surfactants $A$ and $B$ with increasing MAA/HEMA content in aqueous solution (phase boundaries highlighted) B) Conductance measurement for formulations in 3a. Formulations use $0.65 \mathrm{cSt}$ oil, and contain $10 \%$ surfactant by volume. $\mathrm{T}=25^{\circ} \mathrm{C}$. 


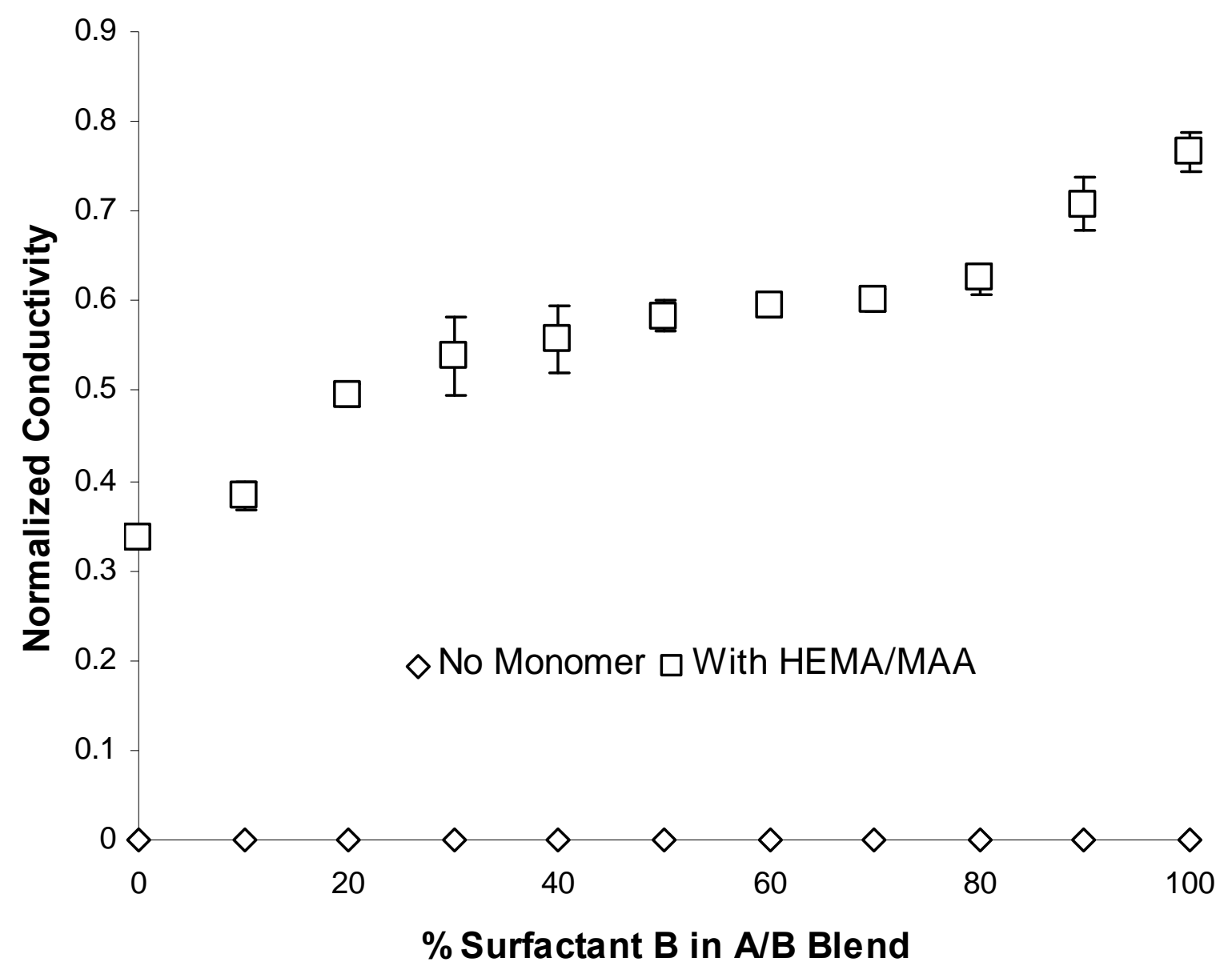

Figure 2-5: Normalized electrical conductivity (conductivity of the microemulsion/ conductivity of aqueous phase) for formulations with and without HEMA/MAA (33\% and $22 \% \mathrm{~V} / \mathrm{V}$ in aqueous solution, respectively). Formulations contain a 1:1 ratio of $0.65 \mathrm{cSt}$ oil and aqueous solution, and contain $10 \%$ surfactant by volume. $T=25^{\circ} \mathrm{C}$.

\subsubsection{Effect of Temperature and $\mathrm{pH}$ on Phase Behaviour}

The phase behaviour of microemulsions containing the aqueous solution with a 4:3:2 volumetric ratio of water: HEMA: MAA, $3 \mathrm{cSt}$ silicone oil, and a 50/50 volumetric blend of surfactants A and $\mathrm{B}$ was characterized at $25{ }^{\circ} \mathrm{C}, 35^{\circ} \mathrm{C}, 45^{\circ} \mathrm{C}$ and $55^{\circ} \mathrm{C}$. Note that the selection of the $50 / 50$ 
blend of surfactant $\mathrm{A} / \mathrm{B}$ was based on the fact that this mixture also produces bicontinuous mixtures with $3 \mathrm{cSt}$ oil (similar studies to that of Figure 2-5), however the mixture is not extremely different from pure A or pure B. The maximum temperature is limited both by the boiling points of the silicone-based components and the heat activated spontaneous polymerization of MAA. Results for the $\mathrm{pH} 2.5$ system are shown in Figure 2-6. While phase behaviour above the critical surfactant concentration, $C^{*}$, was consistent for each system, behaviour below $\mathrm{C}^{*}$ of samples reported as containing two phases could not be characterized by Winsor type. Conductance measurements were therefore used to locate and describe the microemulsion phase. At high aqueous volume fraction, two phase samples were found to contain an upper microemulsion phase that has either oil continuous or bicontinuous characteristics accompanied by excess aqueous solution. At high silicone oil content, samples contained a lower microemulsion phase with bicontinuous characteristics accompanied by an excess oil phase. 


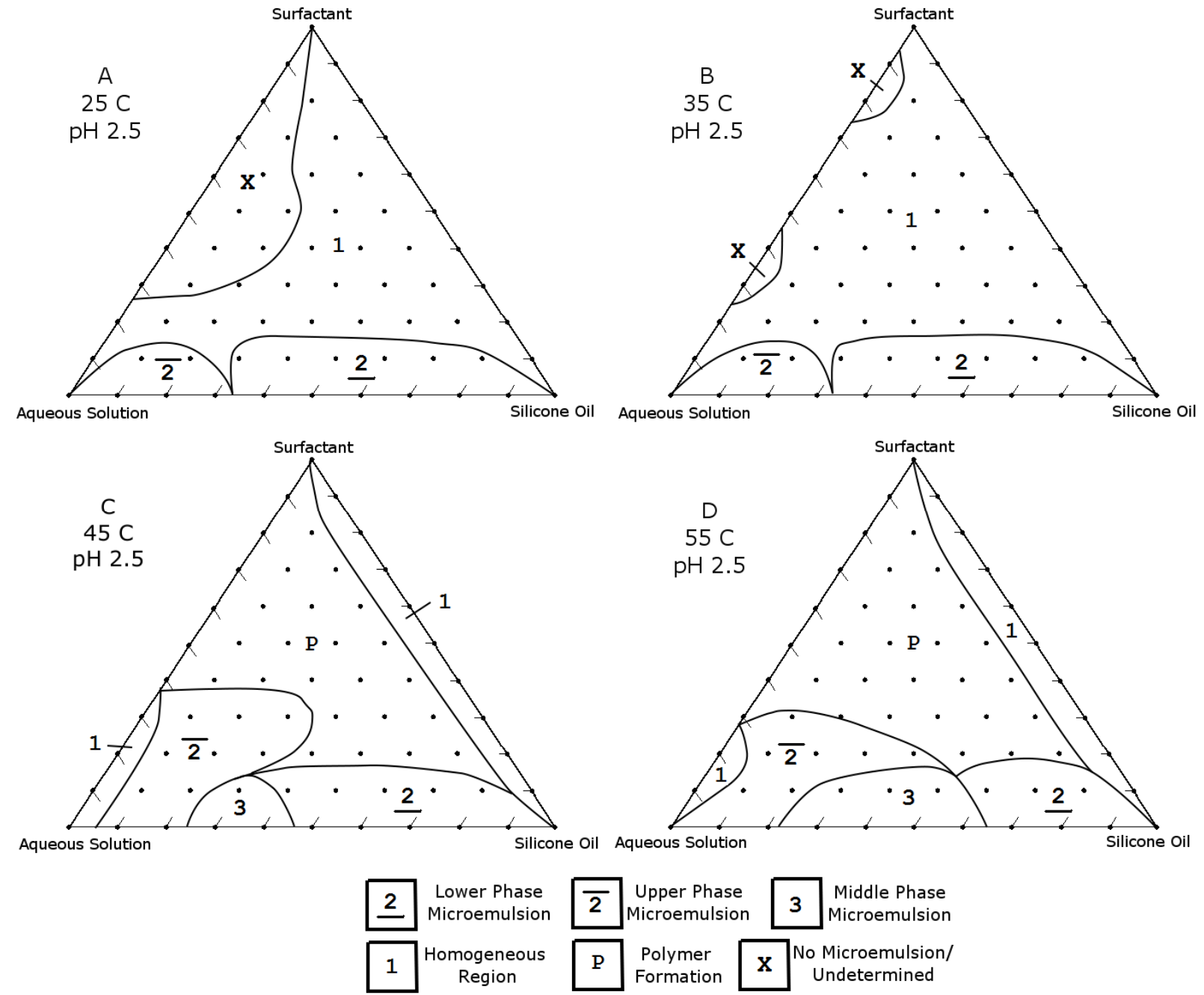

Figure 2-6: Temperature-dependent phase behaviour in pH 2.5, deionized water based system $(n=3)$. Each point represents one sample (10\% increments by volume), with higher resolution ( $2 \%$ increments) near phase boundaries.

At 25 and $35{ }^{\circ} \mathrm{C}$, large regions of homogeneous, optically isotropic liquid were observed, while multiphase regions were observed at below 20\% surfactant and some low silicone oil content compositions. The lowest surfactant concentration necessary to produce a single phase microemulsion $\left(\mathrm{C}^{*}\right)$ was estimated to be $15 \%$ at $25^{\circ} \mathrm{C}$. At 45 and $55^{\circ} \mathrm{C}$, the homogeneous liquid regions are now confined to a smaller range of compositions. The transitions observed at 
higher temperature are consistent with changes predicted by equation $2-2$, particularly that increasing temperature produces a positive shift in HLD. Positive HLD shifts means a greater tendency to incorporate oil and reject water from the microemulsion, which in turns produces a larger region of upper phase microemulsion and a smaller region of lower phase microemulsion. In addition, at the higher temperatures and at nearly all compositions with more than $10 \%$ surfactant, spontaneous polymerization of the monomers occurred (and ensuing polymer precipitation) before well defined microemulsions were observed. Thus, phase behaviour at 45 and $55^{\circ} \mathrm{C}$ cannot be characterized and interpreted conventionally. In samples that formed a homogeneous region at lower temperatures, polymerization at elevated temperature resulted in the formation of thin polymer strands sometimes progressing to substantial solid blocks dispersed throughout the sample. 


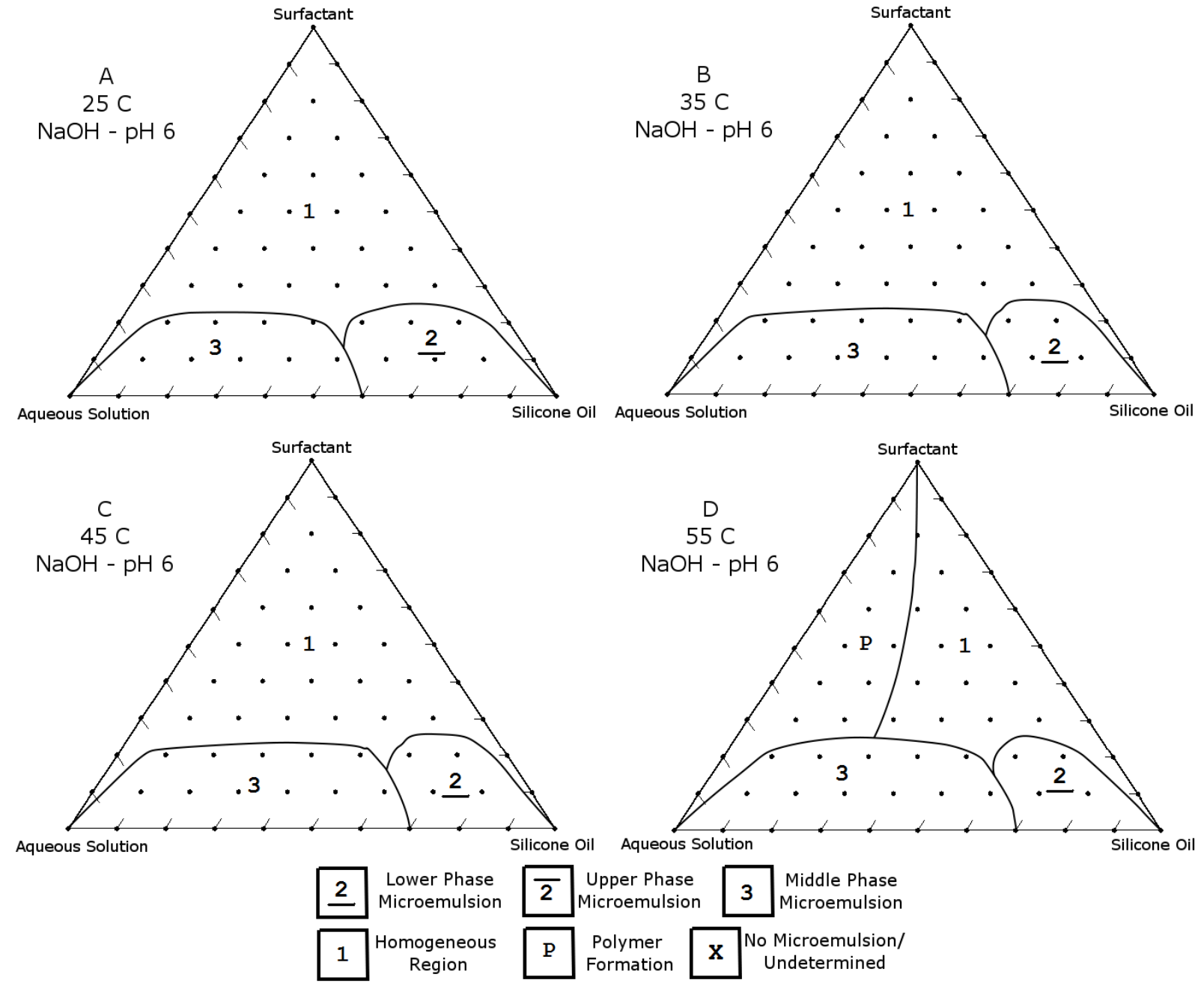

Figure 2-7: Phase behaviour for pH 6 systems adjusted with $\mathrm{NaOH}(\mathrm{n}=3)$. Each point represents one sample (10\% increments by volume), with higher resolution $(2 \%$ increments) near phase boundaries.

We hypothesized that the formation of the polymers at high temperature may have been aided by the relatively low solubility of MAA at $\mathrm{pH} 2.5$. At $\mathrm{pH}$, we expected MAA to be dissociated, thus increasing its solubility and its tendency to remain in the aqueous solution. Raising the aqueous phase $\mathrm{pH}$ to approach the $\mathrm{pKa}$ of MAA led to a reduction in the size of the homogeneous region at $25^{\circ} \mathrm{C}$ and $35^{\circ} \mathrm{C}$, an increase in the region of upper phase 
microemulsion, and a reduction in the region of lower phase microemulsion. These changes are also consistent with the predictions of equation 2-2 and Table 2-3 in that the ionization of MAA at $\mathrm{pH} 6$ produces a positive shift in HLD (a salting out effect) that results in an increase in oil uptake and water rejection from the microemulsion (i.e. larger regions of upper phase microemulsion and smaller regions of lower phase microemulsions). Above the pKa of MAA, further increases in $\mathrm{pH}$ resulted in greater solubilization of the monomers into the aqueous phase, and a large homogeneous microemulsion region even at elevated temperatures. Significantly, the phase behaviour at $\mathrm{pH} 6$ was observed to be temperature-insensitive between 25 and $45{ }^{\circ} \mathrm{C}$, with similar phase diagrams recorded at 25,35 and $45^{\circ} \mathrm{C}$ (Figure 2-7). This observation contrasts with the stronger temperature-dependence seen at $\mathrm{pH} 2.5$. In addition, polymerization and precipitation of reactive monomer components was observed only when temperature was raised to $55^{\circ} \mathrm{C}$ - compared to $45^{\circ} \mathrm{C}$ at $\mathrm{pH} 2.5$. However, the critical surfactant concentration $C^{*}$ for this system was estimated to be $25 \%$, which is significantly higher than at pH 2.5.

\subsubsection{Characterization of the homogeneous region by electrical conductance and microscopy}

Conductance, as an indication of aqueous phase continuity, was measured to further elucidate the structure of the homogeneous regions in the ternary phase diagram. Increasing conductance implies increasing continuity in the aqueous phase, as the volume fraction of the aqueous domains increases. The microemulsion is considered bicontinuous in the isotropic region of non-zero conductance, and in a middle phase with non-zero conductance. ${ }^{55-58}$ 
Conductance was measured at $25^{\circ} \mathrm{C}$ on samples prepared by the incremental dilution of a 1:1 volumetric mixture of $3 \mathrm{cSt}$ silicone oil and surfactant blend (50/50 A:B) with an aqueous or $\mathrm{NaOH}$ solution containing 22\% V/V MAA and 33\% V/V HEMA (Figure 2-8). The measured conductance below $20 \%$ aqueous phase volume fraction was zero, indicating that while homogeneous, this region consists of discrete, disconnected aqueous domains. Connectivity of aqueous domains occurs above aqueous volume fractions of approximately $25 \%$, giving rise to non-zero conductance. However, the normalized conductance was relatively low, suggesting that the proportion of water-continuous channels was relatively low compared to that of oil continuous channels, even for systems containing more than $50 \%$ of the aqueous solution. This may be a consequence of using relatively hydrophobic, water insoluble silicone surfactants, as suggested by the HLD characterization. Due to the complexity of the mixture, results differ from what would be expected in a hydrocarbon oil-water-surfactant system, where the conductance is expected to approach that of the aqueous solution as the water content approaches $100 \%$. At $65 \%$ and $55 \%$ aqueous solution by volume, for $\mathrm{pH} 2.5$ and $\mathrm{pH} 6$, respectively, the samples transition from being a single phase microemulsion into a 2-phase system. Beyond this transition point, the resulting upper phase microemulsion maintains a constant conductance with increasing water content. 


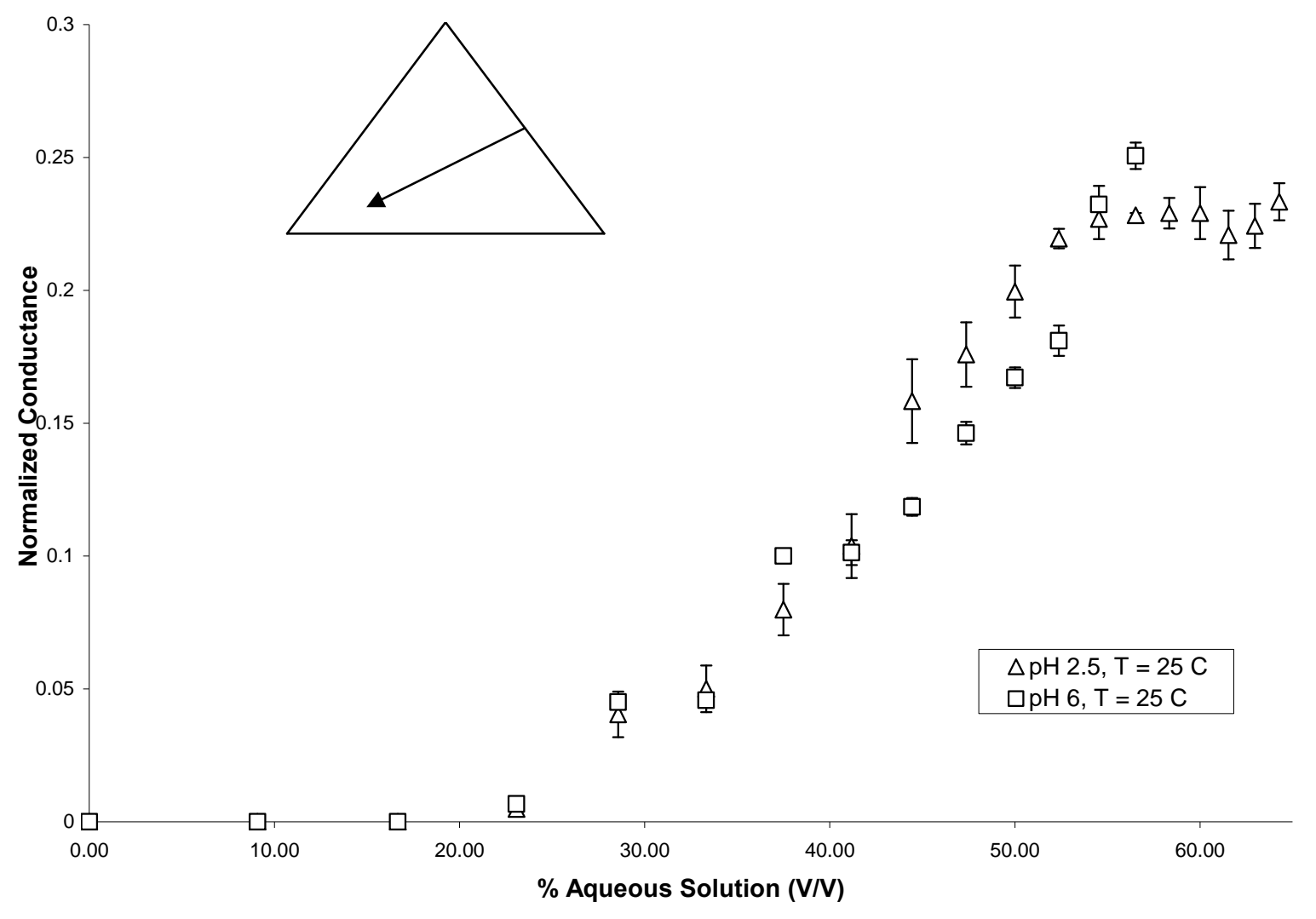

Figure 2-8: Normalized conductance scans of pH 2.5 (triangular points) and pH 6 (square points, $\mathrm{NaOH}$ adjusted) systems. Conductance is normalized to the conductance of the aqueous solution used, and is measured at $25^{\circ} \mathrm{C}(\mathrm{n}=3)$. Conductance is reported for homogeneous formulations only.

Examination of samples with greater than $70 \%$ surfactant and samples along the dilution series used to measure conductance under a cross-polarizing lens found no liquid crystal structures, providing further evidence indicating the formation of a microemulsion structure.

Viscosity measurements are shown in Figure 2-9, for the same dilution series described for conductance measurements. The conductance curve suggests that continuity in the aqueous 
phase is achieved at between $20 \%$ and $30 \%$ aqueous content. This transition coincides with an increase in viscosity, and is consistent with the findings summarized by Gradzielski et al. that the transition into a bicontinuous region is marked by such a viscosity increase ${ }^{59}$. Beyond this point, continued dilution with aqueous solution reduces the viscosity as the droplet-type microemulsion transforms into a bicontinuous structure.

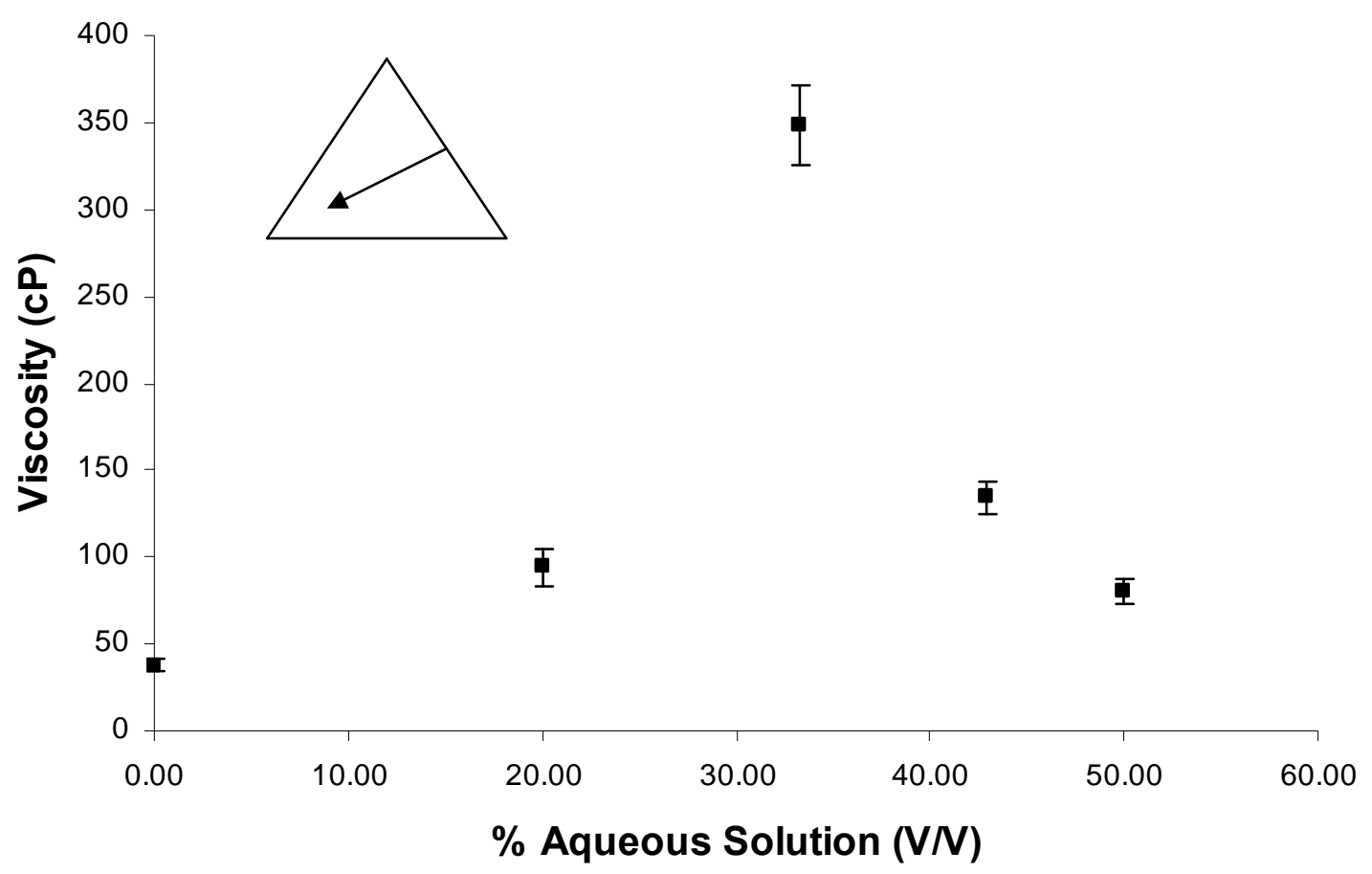

Figure 2-9: Viscosity as measured by falling ball viscometer $(n=3)$ for $3 \mathrm{cSt}$, pH 6 system, $\mathrm{T}=25^{\circ} \mathrm{C}$. 


\subsection{DISCUSSION}

\subsubsection{The hydrophilic-lipophilic nature of silicone oils and silicone alkyl polyether surfactants}

The hydrophilic-lipophilic nature of silicones oils and silicone alkyl polyether surfactants were quantified using the Hydrophilic-Lipophilic Difference empirical equation. Extending the library of known EACNs for oils, surfactant characteristic curvatures $\left(\mathrm{C}_{\mathrm{C}}, \mathrm{C}_{\mathrm{CN}}\right)$, and cosurfactant effects $(\mathrm{f}(\mathrm{A}), \varphi(A))$ will facilitate predicting the phase behaviour of future formulations, or selecting components that will yield desirable phase behaviour. The EACN component of the HLD equation allows us to compare the "net" hydrophobicities of various oils directly. In addition, calculation of EACN from measurements of salinity shifts and correlation with the volume fraction of toluene provided further empirical evidence of the volumetric linear mixing rule described in 2-3. The $0.65 \mathrm{cSt}$ oil (hexamethyl disiloxane, HMDS) essentially contains a C-Si-O-Si-C backbone with 4 methyl side groups, and has an EACN in the reference system of $12.3 \pm 0.7$, indicating a hydrophobicity similar to $n$ - dodecane. The EACN of the 3 cSt oil was $15.0 \pm 0.7$, indicating a hydrophobicity similar to $n$ - pentadecane. Studies on various carbons oils have shown that structural effects such as branching, aromatization, or the presence of oxygen in the backbone reduce the EACN value, offsetting an increase in total carbon/hydrophobic components. For example, the EACNs for benzene, toluene, and dibutyl ether are reported to be 0,1 and 3.4, respectively, much lower than the total number of backbone carbons, molecular weight, or relative mass of hydrophobic and hydrophilic

components would suggest ${ }^{36,60,61}$. However, given the difference in bulk and methyl side 
groups, the relatively low value of EACN for the $3 \mathrm{cSt}$ oil was unexpected. It is important here to note that the $3 \mathrm{cSt}$ oil comprises a mixture of oligomers of different molecular weights, and it is possible that only the smaller molecular weight fraction participates in the microemulsion. This potential segregation of molecular weights should be further considered in future studies.

\subsubsection{Comparison of HDMS and Dodecane}

Comparing the salinity scan for HDMS with a scan for dodecane offers both validation and some insight into the limitations of the EACN concept. While the optimal salinities were similar, microemulsions formed with HDMS maintained a three-phase region over a wider range of salinities than those formed with dodecane. In the context of the HLD framework, the HDMS - SDHS system maintains a middle phase microemulsion over a wider range of HLD values. A possible explanation may lie in an analysis of the two structures, summarized in Table 2-4. While HDMS and dodecane have similar molecular weights, their melting and boiling points differ significantly, indicating higher self-association in linear $n$ - dodecane than in the branched HDMS. The increased chain interactions may present a higher barrier to surfactant-oil association, reducing the ability of a surfactant to penetrate and solubilize the oil, particularly at the level required to form a middle phase microemulsion. Thus, while the EACN allows us to predict the optimal formulation for a middle phase microemulsion, additional molecular information is required to explain the transition points from lower to middle to upper phase microemulsion as a function of formulation variables. 
Table 2-4: Comparison of HDMS and Dodecane properties

\begin{tabular}{c|c|c|c|c|c}
\hline OIL & EACN & $\begin{array}{c}\text { Molecular Weight } \\
(\mathbf{g} / \mathbf{m o l})\end{array}$ & $\begin{array}{c}\text { Density } \\
\left(\mathbf{g} / \mathbf{c m}^{\mathbf{3}}\right)\end{array}$ & $\begin{array}{c}\text { Melting } \\
\text { Point }\left({ }^{\circ} \mathbf{C}\right)\end{array}$ & $\begin{array}{c}\text { Boiling Point } \\
\left({ }^{\circ} \mathbf{C}\right)\end{array}$ \\
\hline HDMS & $12.3 \pm 0.7$ & 162.38 & 0.764 & -59 & 101 \\
Dodecane & 12 & 170.34 & 0.75 & -9.6 & 216 \\
\hline
\end{tabular}

\subsubsection{Validation of the HLD framework in literature}

The HLD framework used here can also be applied to results found through empirical studies in literature. Binks et al. ${ }^{22}$ measured the optimal salinity, $\mathrm{S}^{*}$, for systems containing $0.65 \mathrm{cSt}$ silicone oil (HMDS), water and 2 - ethylhexylsulfosuccinate, sodium salt (AOT). Knowing the $\mathrm{C}_{\mathrm{c}}$ of AOT $(\mathrm{Cc}=2.5)^{49}$, we can use their optimal salinity range of 0.13 to $0.18 \mathrm{M} \mathrm{NaCl}$ to calculate an estimated EACN of $\sim 13-15$, which matches with the upper range for salinity measured in this work. Similarly, HLD calculations using the temperature dependant phase behaviour data for HMDS studied by Silas et al. [21] suggest an EACN of $\sim 14$. On the other hand, a study by Steytler et al. ${ }^{20}$ found that $0.65 \mathrm{cSt}$ silicone oil (HMDS) in an HMDS-waterAOT W/O system yielded droplet sizes and structures similar to systems containing $n$ - heptane, with water solubilization similar to systems containing $n$ - dodecane. 
One of the advantages of using the HLD framework is that it facilitates predicting solubilization. Figure 2-10 presents a comparison between the water to surfactant molar solubilization ratios obtained by Binks et al. ${ }^{22}$ for AOT- $0.65 \mathrm{cSt}$ silicone oil and the solubilization ratios predicted using the $\mathrm{HLD}$ calculated with $\mathrm{EACN}=12.3$ for $0.65 \mathrm{cSt}$. The predicted solubilization ratios were obtained using the HLD-NAC (net-average curvature) equation of state that for water in oil (Type II) microemulsions can be simplified to $1 / R_{w}=H L D / L$, where $R_{w}$ is the equivalent solubilization radius of water, and L is the extended length of the surfactant $\left(10 \AA\right.$ for AOT) ${ }^{48}$, 52 . This radius is transformed into a solubilization ratio using the ratio of volume to surface area for a sphere, and an area per molecule for AOT of $110 \AA^{262}$. Considering that there are no fitting parameters involved, the prediction of water solubilization matches reasonably well with the experimental values. 


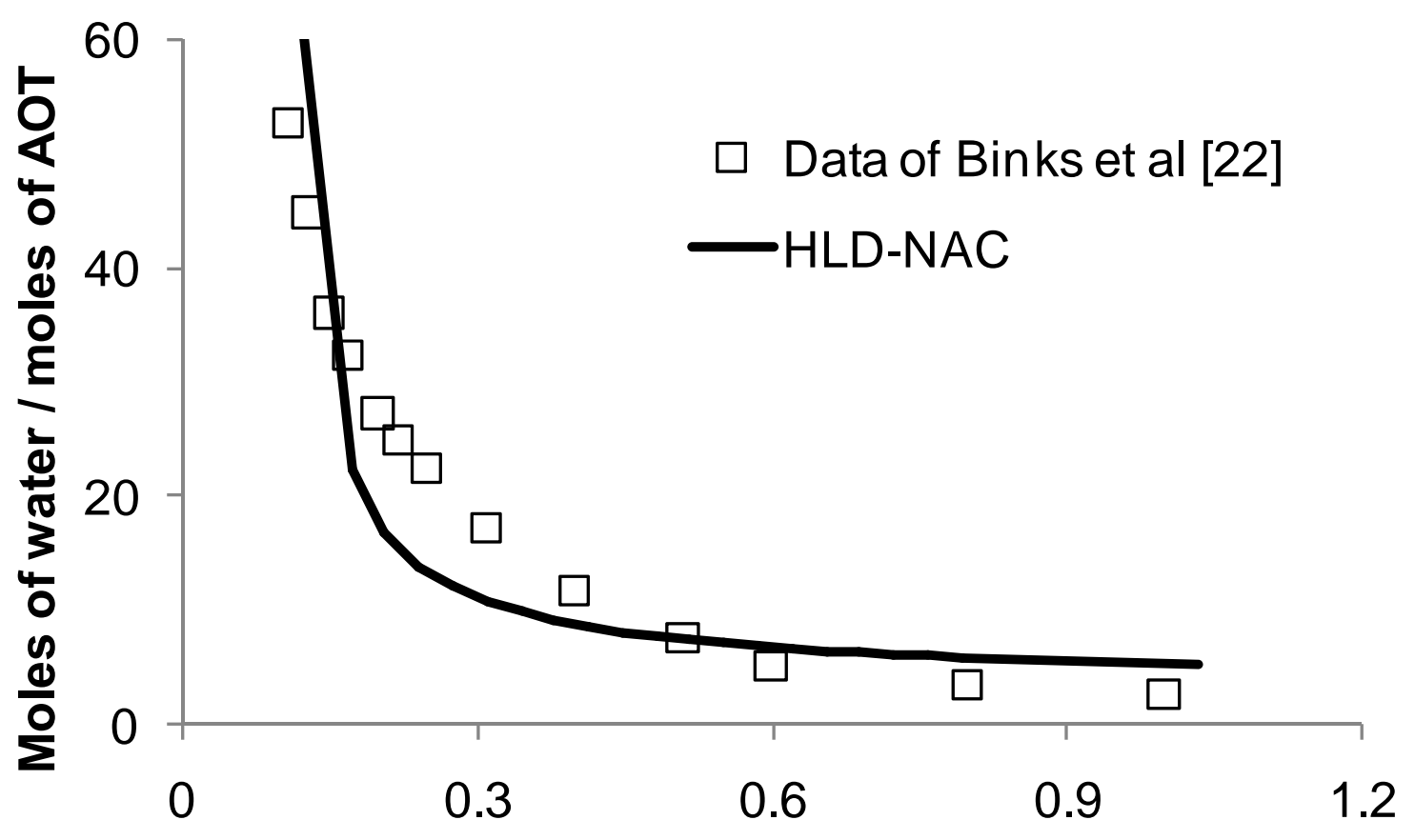

$\mathrm{NaCl}$ concentration, $\mathrm{mol} / \mathrm{L}$

Figure 2-10: Comparison between the molar solubilization ratio of water in AOT-0.65 cSt microemulsions obtained experimentally by Binks et al $^{22}$ and predicted using the HLDNAC model

\subsubsection{Comparison of characteristic curvature and hydrophilic-lipophilic balance}

As previously described, the silicone alkyl polyether surfactant hydrophobicity, and therefore characteristic curvature, is expected to be dependent on the ratio of lauryl to polyether side groups, and the surfactant with the lower ratio yielded a lower characteristic curvature. The calculated values for $\mathrm{C}_{\mathrm{CN}}$ suggest that all surfactants tested partition preferentially into the oil phase, and simple mixing experiments confirm that surfactants A, B and C are not completely miscible with water on their own. It is also relevant to put the calculated values of $\mathrm{C}_{\mathrm{cn}}$ in 
perspective. The largest value of $\mathrm{C}_{\mathrm{cn}}$ for non-ionic surfactants reported before this article was 2.0 for $\mathrm{C}_{12} \mathrm{E}_{4}{ }^{48}$ surfactant. The value of $\mathrm{C}_{\mathrm{cn}}=18.9$ for surfactant A reflects the highly hydrophobic nature of surfactant A.

To add further context to the calculated values for characteristic curvature, we can compare these directly their hydrophilic-lipophilic balance (HLB). The HLB of a surfactant is based on the ratio of the mass of the hydrophilic region to the total mass, providing a link between hydrophobicity and molecular structure. Figure 2-11 presents the relationship between HLB and $\mathrm{C}_{\mathrm{cn}}$ for alkane alcohol ethoxylated $\left(\mathrm{C}_{\mathrm{n}} \mathrm{E}_{\mathrm{j}}\right)$ surfactants, nonylphenol ethoxylated $\left(\mathrm{NPE}_{\mathrm{j}}\right)$ surfactants and the silicone surfactants studied in the current work. 


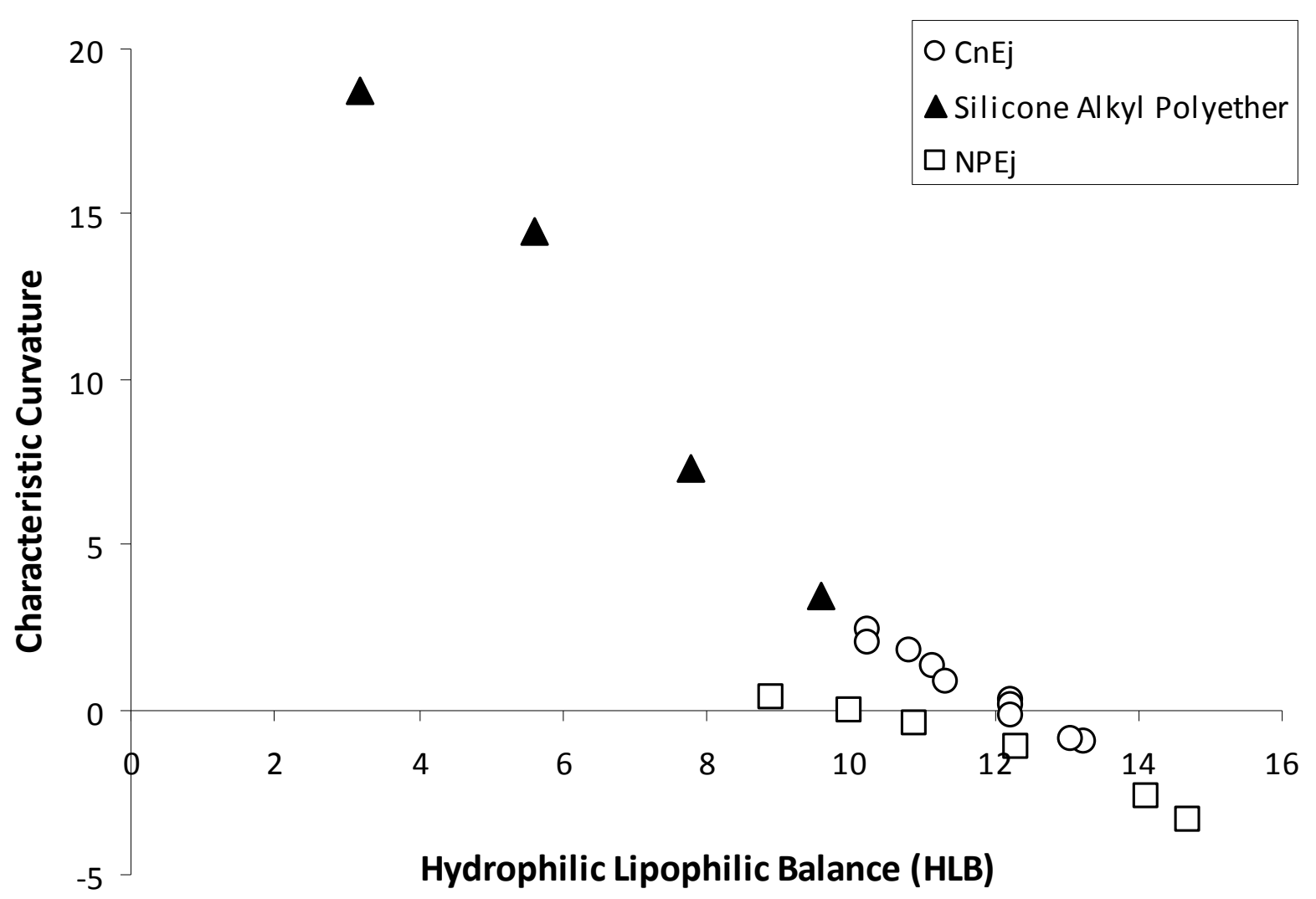

Figure 2-11: HLB vs. $C_{c n}$ for selected alkane alcohol ethoxylated $\left(C_{n} E_{j}\right)$, nonylphenol ethoxylated $\left(\mathrm{NPE}_{\mathrm{j}}\right)$ and silicone alkyl polyether surfactants (J208-X12). HLB and $\mathrm{C}_{\mathrm{CN}}$ values for alcohol ethoxylated and nonylphenol ethoxylated surfactants derived from ${ }^{48}$.

It is interesting to note that while the HLB value is based on molecular structure, it does not explicitly account for conformation, interfacial behaviour of the surfactant molecule, or formulation variables in general. The correlation between $\mathrm{HLB}$ and $\mathrm{C}_{\mathrm{cn}}$ suggested by Figure 2-11 indicates a link between the structure dependent HLB and the semi-empirical $\mathrm{C}_{\mathrm{cn}}$ for nonionic surfactants, a property that has been previously reported ${ }^{49}$. More importantly, the trends in Figure 2-11 show that the correlation for silicone alkyl polyether surfactants is comparable to that of hydrocarbon based non-ionic surfactants. 


\subsubsection{Phase Behaviour and Polymerization}

The calculated EACN of 15.0 for $3 \mathrm{cSt}$ silicone oil in an SDHS-oil-water system suggests that despite its bulk and methyl group density, it remains possible to bridge the gap between the oil and aqueous phases by using the HLD equation to select appropriate surfactants and organic water-soluble co-solvents. The silicone-based surfactants at our disposal are certainly capable of bridging the hydrophobicity gap, according to the HLD equation. The introduction of MAA and HEMA as aqueous co-solvents produces a significant negative shift in the HLD value. The mixture of 4:3:2 water-HEMA-MAA by volume behaves less like water and more like a nonaqueous solvent. While a systematic study of the effect of non-aqueous solvents on HLD shifts is still missing, it is pertinent to mention the work of Schubert et al. ${ }^{63}$ with microemulsions of formamide-AOT-styrene (EACN 2) - NaBr. Using the HLD equation, one would predict an optimal salinity of $0.2 \% \mathrm{NaBr}$ in aqueous solution; however, the optimal salinity with formamide reported by Schubert et al. is close to $11 \% \mathrm{NaBr}$. This suggests that the replacement of water with formamide produces a shift in HLD of approximately -4. A much larger shift in HLD seemed to be attained with mixtures of HEMA and MAA, perhaps indicating the mixture used here is less hydrophilic than formamide.

In the ternary phase diagram of aqueous phase (water-HEMA-MAA) - surfactant $(\mathrm{A}+\mathrm{B})$ - oil (3cSt PDMS) the changes in the phase behaviour with increasing temperature and $\mathrm{pH}$ are consistent with the behaviour predicted by the HLD equation. The positive HLD shifts associated with the increase in temperature are believed to be related to the weakening of 
hydrogen bonds ${ }^{48}$. The positive HLD shift observed with increasing electrolyte concentration (the ionization of MAA at $\mathrm{pH}$ 6) is associated with the salting out of the surfactant and MAA.

An increase in temperature for the systems at $\mathrm{pH} 2.5$ appears to facilitate polymerization of the monomers and aggregation of the surfactants. While spontaneous polymerization of inhibited MAA is possible at elevated temperatures, spontaneous polymerization at $35-40{ }^{\circ} \mathrm{C}$ was unexpected. Polymerization at elevated temperatures occurred despite the presence of an inhibitor. The inhibitor, monomethyl ether of hydroquinone (MEHQ) is used in commercial MAA and in HEMA to retard polymerization during storage. MEHQ effectiveness is known to decrease at elevated temperatures, in aqueous solution, and with depressed dissolved oxygen content; however, this effect is not expected at atmospheric pressure within the range of temperatures examined in this study ${ }^{64-66}$. Experimental results suggest that MEHQ, a free radical scavenger soluble in both water and organic solvents, possibly partitioned out of the aqueous phase in these microemulsion systems.

\subsection{CONCLUDING REMARKS}

While previous microemulsion formulation has focused primarily on empirical approaches, or trial and error, the HLD framework is intended to provide a mathematical approach based on formulation variables and properties of components ${ }^{36,41}$. In this work, it has been shown that the HLD framework can be applied to formulations containing silicone oils and surfactants. The HLD equations were used to characterize silicone oils by an EACN, silicone alkyl polyether 
surfactants by a characteristic curvature, and mixtures of MAA and HEMA in aqueous solution by their effects on HLD shifts. These findings provide a quantifiable and consistent basis for formulating and comparing reactive, silicone-based microemulsions. Previous results of empirical approaches in the literature have indicated difficulties in microemulsion formulation and solubilization with unmodified silicone oils, attributed to an "enhanced" hydrophobic behaviour $19,20,22,25,62$. This behaviour has been quantified in order to provide context for those findings, and have presented a general method for characterizing silicone oils and derivatives in the future. In addition, the EACN characterization has been validated by comparing HDMS to dodecane. The approach to reactive silicone microemulsion formulation presented here provides a basis for work linking silicone microemulsion structure to the components used in formulation.

The formation of a reactive, bicontinuous microemulsion containing MAA and HEMA, confirmed by conductivity measurement, has also been demonstrated, and its phase behaviour has been investigated with respect to composition, temperature and $\mathrm{pH}$. Characterization indicates a link between increasing monomer content and a higher required surfactant hydrophobicity, elaborating on previous studies describing the effects of replacing water with non-aqueous solvent in a microemulsion ${ }^{54}$. The bicontinuous structures developed here are intended to provide a template for polymerization or other reactions requiring interpenetrating aqueous and silicone oil channels. 


\subsection{REFERENCES}

1. Hoar, T. P.; Schulman, J. H. Nature 1943, 3847, 102-103.

2. Danielsson, I.; Lindman, B. Colloids and Surfaces 1981, 4, 391-392.

3. Winsor, P. A. Solvent Properties of Amphiphilic Compounds; Butterworths: London, 1954; .

4. Winsor, P. A. Transactions of the Faraday Society 1948, 376-398.

5. Salager, J.; Antón, R. E.; Sabatini, D. A.; Harwell, J. H.; Acosta, E. J.; Tolosa, L. I. J Surfact Deterg 2005, 1, 3-21.

6. Holmberg, K. Microemulsions in Biotechnology. In Industrial Application of Microemulsions; Solans, C., Kunieda, H., Eds.; Marcel Dekker: New York, 1997; pp 69.

7. Fredon, L.; Mallard, C. Ann. Dermatol. Venereol. 2007, 3 C2, 2S30-2S36.

8. Patravale, V. B.; Mandawgade, S. D. Int. J. Cosmetic Sci. 2008, 1, 19-33.

9. Lawrence, M. J.; Rees, G. D. Advanced Drug Delivery Reviews 2000, 1, 89-121.

10. Carrión, F. J. Boletin Intexter del Instituto de Investigacion Textil y de Cooperacion Industrial 2007, 131, 17-25.

11. Hou, A.; Chen, S. J. Dispersion Sci. Technol. 2010, 1, 102-107.

12. Parvinzadeh, M.; Hajiraissi, R. Journal of Surfactants and Detergents 2008, 4, 269-273.

13. Hashem, M.; Ibrahim, N. A.; El-Shafei, A.; Refaie, R.; Hauser, P. Carbohydr. Polym. 2009, 4, 690-703.

14. Zhang, D.; Jiang, X.; Yang, C. J Appl Polym Sci 2003, 13, 3587-3593.

15. Zhang, D.; Jiang, X.; Yang, C. J Appl Polym Sci 2005, 1, 347-352.

16. Xu, Y.; Liu, Y.; Zhao, N.; Yuan, C. Huagong Xuebao/Journal of Chemical Industry and Engineering (China) 2006, 6, 1464-1467.

17. Guerrero-Ramírez, L. G.; Nuño-Donlucas, S. M.; Cesteros, L. C.; Katime, I. Materials Chemistry and Physics 2008, 3, 1088-1092.

18. Sharma, S. C.; Tsuchiya, K.; Sakai, K.; Sakai, H.; Abe, M.; Komura, S.; Sakamoto, K.; Miyahara, R. Langmuir 2008, 15, 7658-7662.

19. Messier, A.; Schorsch, G.; Rouviere, J.; Tenebre, L. Trends in Colloid and Interface Science III 1989, 249-256.

20. Steytler, D. C.; Dowding, P. J.; Robinson, B. H.; Hague, J. D.; Rennie, J. H. S.; Leng, C. A.; Eastoe, J.; Heenan, R. K. Langmuir 1998, 13, 3517-3523.

21. Silas, J. A.; Kaler, E. W.; Hill, R. M. Langmuir 2001, 15, 4534-4539.

22. Binks, B. P.; Dong, J.; Rebolj, N. Phys. Chem. 1999, 9, 2335-2344.

23. Strey, R. Curr. Opin. Colloid Interface Sci. 1996, 402-410. 
24. Gee, R. P. Colloids and Surfaces A: Physicochemical and Engineering Aspects 1998, 1-3, 91-101.

25. John, A. C.; Uchiyama, H.; Nakamura, K.; Kunieda, H. Journal of Colloid and Interface Science 1997, 2, 294-299.

26. Liu, J.; Huang, S.; Wu, S. Key Engineering Materials 2008, 497-500.

27. Liu, J.; Wu, S.; Huang, S.; Cong, P.; Mi, Y. Iranian Polymer Journal (English Edition) 2009, 2, 159-166.

28. Sharma, S. C.; Tsuchiya, K.; Sakai, K.; Sakai, H.; Abe, M.; Miyahara, R. Journal of Oleo Science 2008, 12, 669-673.

29. Hill, R. M. Current Opinion in Colloids and Interface Science 2002, 5-6, 255-261.

30. O'Lenick, A. J. Journal of Surfactants and Detergents 2000, 3, 387-393.

31. Gašperlin, M.; Rebolj, N.; Spiclin, P.; Kristl, J. Acta Pharmaceutica 2002, 2, 101-111.

32. Kumar, A.; Uddin, M. H.; Kunieda, H.; Furukawa, H.; Harashima, A. J. Dispersion Sci. Technol. 2001, 2-3, 245-253.

33. Li, X.; Washenberger, R. M.; Scriven, L. E.; Davis, H. T.; Hill, R. M. Langmuir 1999, 7, 2278-2289.

34. Li, X.; Washenberger, R. M.; Scriven, L. E.; Davis, H. T.; Hill, R. M. Langmuir 1999, 7 , 2267-2277.

35. Salager, J. L.; Morgan, J. C.; Schechter, R. S.; Wade, W. H.; Vasquez, E. Soc Pet Eng AIME $J$ 1979, 2, 107-115.

36. Bouton, F.; Durand, M.; Nardello-Rataj, V.; Serry, M.; Aubry, J. Colloids Surf. Physicochem. Eng. Aspects 2009, 1-3, 142-147.

37. Kiran, S. K.; Acosta, E. J.; Moran, K. J. Colloid Interface Sci. 2009, 1, 304-313.

38. Nardello, V.; Chailloux, N.; Poprawski, J.; Salager, J. -L.; Aubry, J. -M. Polym. Int. 2003, 4, 602-609.

39. Pakpayat, N.; Nielloud, F.; Fortuné, R.; Tourne-Peteilh, C.; Villarreal, A.; Grillo, I.; Bataille, B. European Journal of Pharmaceutics and Biopharmaceutics 2009, 2, 444-452.

40. Poprawski, J.; Catté, M.; Marquez, L.; Marti, M. -J.; Salager, J. -L.; Aubry, J. -M. Polym. Int. 2003, 4, 629-632.

41. Witthayapanyanon, A.; Harwell, J. H.; Sabatini, D. A. J. Colloid Interface Sci. 2008, 1, 259266.

42. Chow, P. Y.; Gan, L. M. Microemulsion polymerizations and reactions. In Polymer Particles; Okubo, M., Ed.; Springer-Verlag: Berlin, 2005; Vol. 175, pp 257.

43. Chew, C. H.; Li, T. D.; Gan, L. H.; Quek, C. H.; Gan, L. M. Langmuir 1998, 21, 6068-6076.

44. O’Donnell, J.; Kaler, E. W. Macromolecules 2008, 16, 6094-6099.

45. Gelest, I. Silanes, Silicone, Metal-Organic Compounds by Gelest, Inc. http://www.gelest.com/. 
46. Siltech Corporation Siltech organo functional silicone compounds and related specialties. http://www.siltechcorp.com/index.php?option=com_content\&task=view\&id=42\&Itemid $=33$ (accessed 7/24/2009, 2009).

47. Szekeres, E.; Acosta, E.; Sabatini, D. A.; Harwell, J. H. J. Colloid Interface Sci. 2006, 1, 222-233.

48. Acosta, E. J. Colloids Surf. Physicochem. Eng. Aspects 2008, 1-3, 193-204.

49. Acosta, E. J.; Yuan, J. S.; Bhakta, A. S. Journal of Surfactants and Detergents 2008, 2, 145158.

50. Baran Jr., J. R.; Pope, G. A.; Wade, W. H.; Weerasooriya, V.; Yapa, A. J. Colloid Interface Sci. 1994, 1, 67-72.

51. Acosta, E. J.; Bhakta, A. S. Journal of Surfactants and Detergents 2009, 1, 7-19.

52. Kiran, S. K.; Acosta, E. J. Ind. Eng. Chem. Res. 2010, 7, 3424.

53. Rico, I.; Lattes, A. JCIS 1984, 1, 285.

54. Schubert, K. -V.; Lusvardi, K. M.; Kaler, E. W. Colloid and Polymer Science 1996, 9, 875.

55. Lim, W. H. J Surfact Deterg 2006, 4, 349-355.

56. Meier, W. Colloid Polym. Sci. 1997, 6, 530-536.

57. Laguës, M.; Sauterey, C. J. Phys. Chem. 1980, 26, 3503-3508.

58. Clausse, M.; Peyrelasse, J.; Heil, J.; Boned, C.; Lagourette, B. Nature 1981, 5834, 636-638.

59. Gradzielski, M.; Hoffmann, H. Rheological properties of microemulsions. In Handbook of Microemulsion Science and Technology; Kumar, P., Mittal, K. L., Eds.; CRC: 1999; pp 357-386.

60. Queste, S.; Salager, J. L.; Strey, R.; Aubry, J. M. J. Colloid Interface Sci. 2007, 1, 98-107.

61. Szekeres, E.; Acosta, E.; Sabatini, D. A.; Harwell, J. H. Langmuir 2004, 16, 6560-6569.

62. 1. Rosen, M. J. Surfactants and Interfacial Phenomena, 3rd edition; John Wiley and Sons: New York, 2004.

63. Schubert, K. -V.; Kaler, E. W. Phys. Chem. 1996, 190-205.

64. Becker, H.; Vogel, H. Chem. Eng. Technol. 2006, 10, 1227-1231.

65. Cutié, S. S.; Henton, D. E.; Powell, C.; Reim, R. E.; Smith, P. B.; Staples, T. L. J Appl Polym Sci 1997, 3, 577-589.

66. Li, R.; Joseph Schork, F. Industrial and Engineering Chemistry Research 2006, 9, 30013008 . 


\section{SOLUBILITY AND SOLUBILIZATION: EVALUATING PARAMETERS AND DEVIATIONS IN THE HLD FRAMEWORK}

\subsection{INTRODUCTION}

The HLD framework in conjunction with phase behaviour studies provided a basis for formulating and understanding silicone microemulsions with desirable characteristics. In this chapter, we examine in detail features of the phase behaviour analysis and HLD parameters for the formulations developed and presented in chapter 2. Of particular interest are the hydrophilic linker concept, the effects of monomer solubility on microemulsion formation and the counterion effect with regards to nonionic surfactants. The linker effect and monomer solubility may be partly accounted for in the HLD framework through the co-surfactant terms $f(A)$ and $\varphi(A)$, while the counter ion effect for nonionic surfactants is accounted for in the parameter $b$ for the salinity term $b \times S$. 


\subsection{AQUEOUS MONOMERS AND THE LINKER EFFECT}

The phase behaviour studies in chapter 2 indicate that the introduction of MAA and HEMA to the aqueous solution causes a significant negative shift in HLD consistent with effects of a hydrophilic co-surfactant. Thus we hypothesize that:

If the monomers play a role as hydrophilic linkers, then this effect can be quantified through the HLD framework for co-surfactants.

\section{\begin{tabular}{l|l} 
Aqueous Phase & Silicone Phase
\end{tabular}}

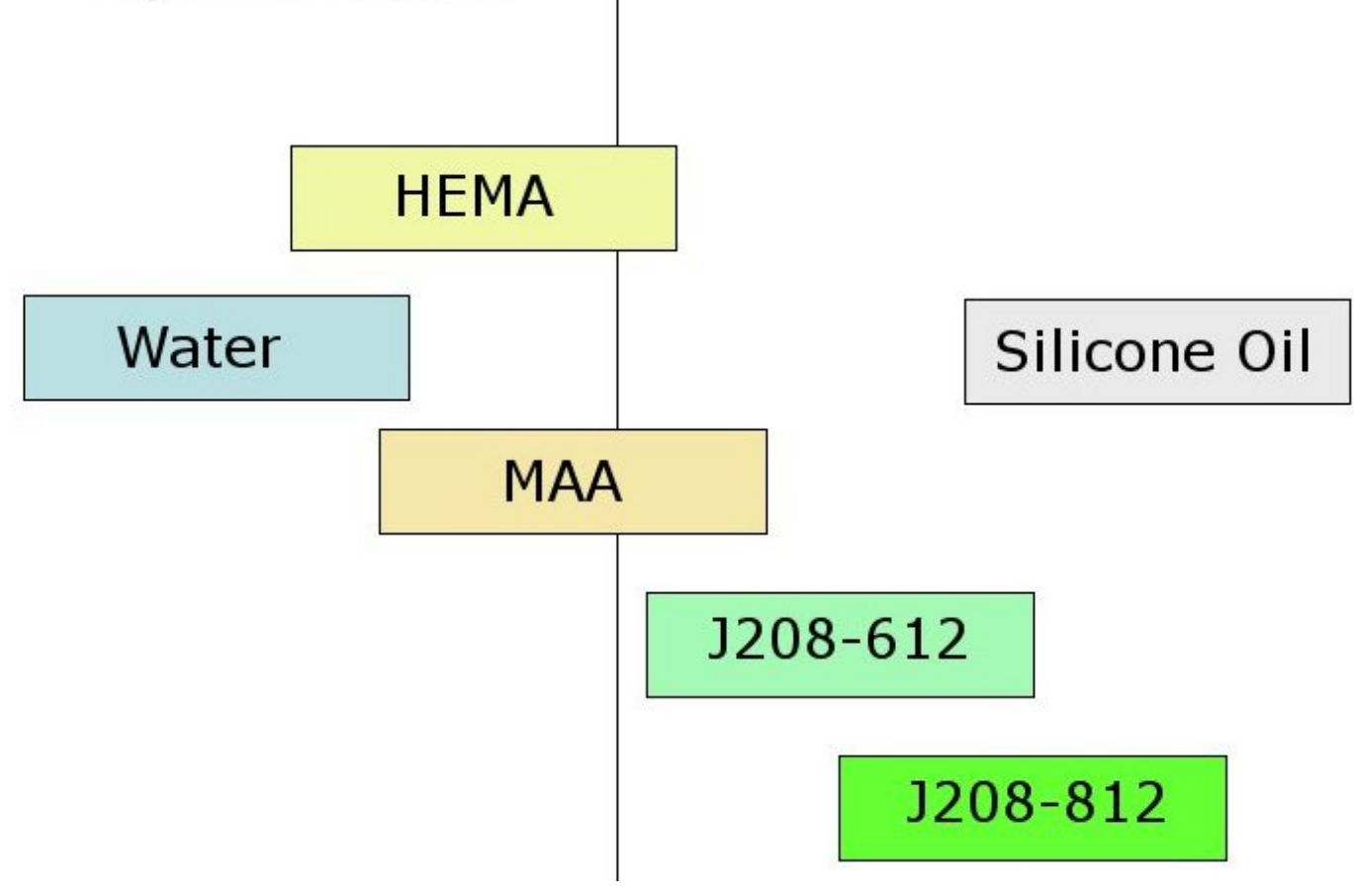

Figure 3-1: Schematic representation of proposed linker effect in silicone microemulsion based on aqueous solubility/miscibility. The central line represents the oil-water interface. The locations of each box represent the theoretical overlap between molecules of each species near the interface, reflecting the extension of interfacial order into each phase. 
In order to evaluate the contribution of HEMA and MAA to the linker effect, a linking scheme was proposed for testing on the basis of aqueous solubility/miscibility, shown in Figure 3-1. The linker effect is further described in section 1.2.3. Samples containing surfactant blend, nonreactive $3 \mathrm{cSt}$ silicone oil and aqueous solution at pH 6 were formulated according to Table 3-1. Previous results had shown that the 50:50 surfactant blend does not differ much from pure A or pure B. Table 3-1 illustrates that when components are omitted, the formulation fails to form a microemulsion. Many of the results are easily predicted, in that HEMA aids MAA solubility, and the surfactant blend is not miscible with water. However, the results suggest that both MAA and the silicone oil are also necessary links in stabilizing the microemulsion and preventing aggregation of the surfactant molecules in water.

Table 3-1: Selected formulation specifications for evaluation of the linker effect. Samples were formed from equal parts aqueous, oil and surfactant and equilibrated at $25^{\circ} \mathrm{C}$ for 14 days. Percentages are by volume. The surfactant blend contains equal parts surfactants $A$ and $B$.

\begin{tabular}{cccccc}
\hline $\begin{array}{c}\text { NaOH } \\
\text { Solution }\end{array}$ & HEMA & MAA & $\begin{array}{c}\text { Surfactant } \\
\text { Blend }\end{array}$ & $\begin{array}{c}\text { 3 cSt } \\
\text { Silicone Oil }\end{array}$ & \multicolumn{1}{l}{ Result } \\
\hline $14.7 \%$ & $11.0 \%$ & $7.3 \%$ & $33.3 \%$ & $33.3 \%$ & Homogeneous \\
$14.7 \%$ & $18.3 \%$ & - & $33.3 \%$ & $33.3 \%$ & Polymer Precipitation within 14 days \\
$14.7 \%$ & - & $18.3 \%$ & $33.3 \%$ & $33.3 \%$ & Polymer Precipitation within 2 days \\
- & $22.2 \%$ & $11.1 \%$ & $33.3 \%$ & $33.3 \%$ & Some Precipitation within 14 days \\
$33.3 \%$ & - & - & $33.3 \%$ & $33.3 \%$ & Turbid, low viscosity dispersion \\
$50 \%$ & - & - & $50 \%$ & - & High viscosity, turbid, gel-like \\
& & & & - & dispersion \\
$22.2 \%$ & $16.7 \%$ & $11.1 \%$ & $50 \%$ & - & Turbid, high viscosity dispersion \\
\hline
\end{tabular}


In addition to the formulations described in Table 3-1, formulations were generated with combinations of surfactants Y, Z, and a hydrophilic silicone polyether surfactant, Silsurf D212CG $(\mathrm{HLB}=10.4)$. Systems containing mixtures of water, surfactant blends and silicone oil (no HEMA or MAA) did not form a single phase. Combinations of water soluble/hydrophilic and water insoluble/hydrophobic surfactants led to the formation of Type I and II systems. While the water-soluble silicone polyethers and insoluble silicone alkyl polyethers are miscible on their

own, the introduction of oil and/or water caused the surfactants to partition into their preferred environment. Combinations of relatively hydrophilic surfactants did not lead to microemulsion formation, and combinations of relatively hydrophobic surfactants led to the formation of a turbid gel.

\subsection{MONOMER SOLUBILITY IN AQUEOUS SOLUTION}

While the HLD framework accounts for formulation variables such as temperature, the nature of the oil, the natures of the surfactant/co-surfactants, and the nature of the counter-ion, pseudoternary phase diagrams were still required to evaluate the effect of MAA solubility limits on microemulsion formation and behaviour. The importance of solubility is evident in that the HLD assumes the formation of a microemulsion from an equilibrated mixture of immiscible liquids with a mediating surface active agent. The HLD equations do not explicitly account for the formation of precipitates, phase separation in either the oil or aqueous components, or the formation of lamellar and liquid crystal phases, necessitating the use of ternary phase diagrams to explore formulation phase behaviour over the complete range of compositions. 


\subsubsection{Estimating $\varphi(A)$ for HEMA and MAA in aqueous solution}

The co-surfactant contribution to the nonionic HLD equation, $\varphi(A)$, has yet to be studied for HEMA and MAA. However, it can be estimated from the calculated HLD value at $\mathrm{pH} 6$ in Table 2-3 for the formulation described in Figure 2-4 (45\% oil, 45\% aqueous, 10\% surfactant), so that $\mathrm{HLD}=16.3$. According to Figure 2-7 and Figure 2-4, this formulation forms a middle phase microemulsion at a monomer concentration of $2.4 \mathrm{~mol} / \mathrm{L}$ on a total volume basis. The function $\varphi(A)$ is approximated in literature as linear with respect to co-surfactant concentration ${ }^{1-}$ ${ }^{3}$ so that:

\section{3-1: $\varphi(A)=m \times A$}

where $m$ is an empirically derived constant associated with the co-surfactant and $A$ is the cosurfactant concentration based on total volume. In order to evaluate 3-1, we make the simplifying assumption that the addition of monomer causes a reduction in HLD from 16.3 to 0 at $25^{\circ} \mathrm{C}$, the optimal formulation. This assumption is based on the transition from upper to middle phase microemulsions described in section 2.3.4. Hence $m=6.8 \mathrm{~L} / \mathrm{mol}$, and $\varphi(A)=6.8$ $\times$ A for a 3:2 volumetric mixture of HEMA and MAA.

However, as described in section 2.2.2, it is likely that the formulation does not reach the optimal concentration, but rather that it is near the upper HLD bound to the three-phase region. Thus this estimate of $\varphi(A)$ in turn represents an upper bound, such that $\mathrm{m} \leq 6.8$. In order to improve the accuracy of the estimate, the maximum monomer concentration that still produces a 
middle phase microemulsion would be required. However, this point is beyond the solubility limit for monomers in this formulation. In addition, the linear approximation for $\varphi(A)$ may be restricted to co-surfactant concentration below the aqueous solubility limit. From the analysis above and the analysis in section 2.3.4, one could consider the function $\varphi(A)$ to represent the effect of replacing water with a non-aqueous solvent, such that $\varphi(A)=0$ when the aqueous solvent is $100 \%$ water. As the co-surfactant content is increased, the aqueous phase becomes less water-like. If the co-surfactant content is increased beyond the solubility limit, it precipitates, and the HLD framework is no longer applicable. As the co-surfactant content continues to increase, it replaces water as the continuous phase.

\subsubsection{Monomer solubility, phase separation and precipitation}

Precipitation of MAA and HEMA occurred through two mechanisms: Aqueous phase separation and spontaneous polymerization. The former case can be illustrated by examining conductivity measurements for the $\mathrm{pH} 2.5$ system above $70 \%$ aqueous content, continuing along the dilution line used in Figure 2-8. At this point, the homogeneous, bicontinuous microemulsion splits into an upper phase microemulsion with excess aqueous solution, with conductivity for the upper and lower phases shown in Figure 3-2.

As the figure illustrates, the microemulsion approaches a constant conductance below $50 \%$ of the original aqueous solution conductance. By contrast, the conductance of the excess aqueous solution is approximately 2.5 times the original aqueous solution conductance. As a point of 
reference, the conductivity of the distilled water is $<5 \%$ of the aqueous solution conductivity, indicating that the monomers, MAA and HEMA, are the primary contributors to charge transfer. Figure 3-2 further illustrates that the excess aqueous phase is not the original aqueous solution, but rather contains a much higher concentration of conductive elements.

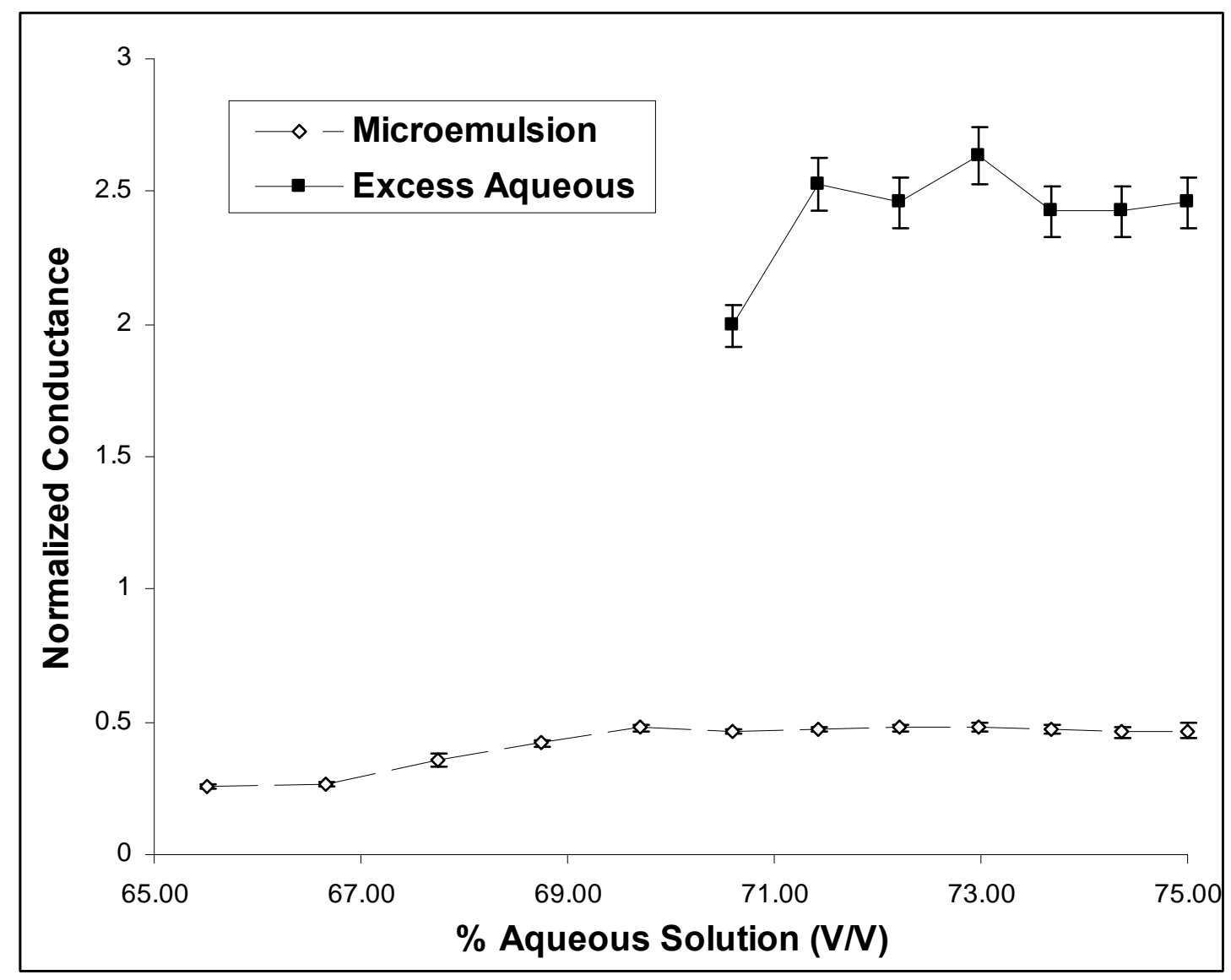

Figure 3-2: Normalized conductance scans for microemulsion and excess aqueous phase in pH 2.5 formulation. Conductance is normalized to the conductance of the aqueous solution used, and is measured at $25^{\circ} \mathrm{C}(\mathrm{n}=3)$. Points below $70 \%$ aqueous content are homogeneous microemulsions. 
In the second case, solid precipitates were observed as a result of surfactant-water interactions and polymerization of the aqueous monomers at elevated temperature in the $\mathrm{pH} 2.5$ system. At 45 and $55^{\circ} \mathrm{C}$, samples that contained excess aqueous solution at lower temperatures yielded a solid layer of polymer at the base of the vial. In samples that formed a homogeneous region at lower temperatures, polymerization at elevated temperature resulted in the formation of thin polymer strands sometimes progressing to substantial solid blocks dispersed throughout the sample. Observations from this region can be further sub-categorized. At low surfactant content (10-20\%), the polymer contracted into a flexible solid when cooled, as shown in Figure 3-3 (middle). At low aqueous content (20-30\%), these strands settled to the base of the vial as a turbid, viscous dispersion once the samples were allowed to cool, as shown in Figure 3-3 (right). In addition to polymer precipitate, multiphase samples with low oil content developed a layer of turbid surfactant aggregate, as shown in Figure 3-3 (left).

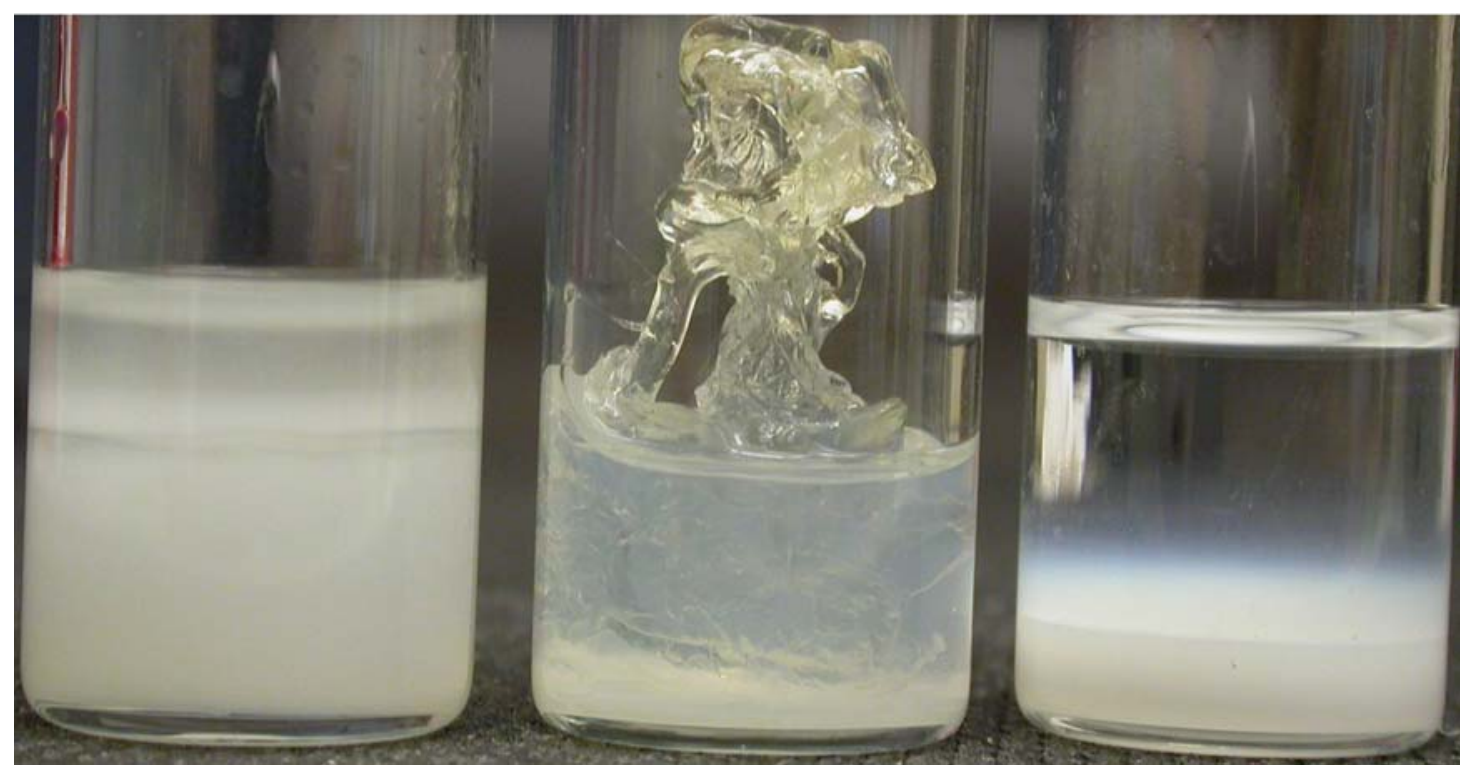

Figure 3-3: Polymerization and settling of reactive monomer components and aggregation of surfactants with (Left) $60 \%$ surfactant and $40 \%$ pH 2.5 aqueous phase; (Middle) $10 \%$ 
surfactant and 40\% pH 2.5 aqueous phase; (Right) $40 \%$ surfactant and $20 \%$ pH 2.5 aqueous phase.

As described in section 2.3.5, we hypothesized that spontaneous polymerization at high temperature may have been aided by the relatively low solubility of MAA at $\mathrm{pH} 2.5$. At $\mathrm{pH}$, the MAA is expected to dissociate, thus increasing its solubility as a monomer in the aqueous solution.

\subsubsection{Discussion: Solubility and the Solubilization}

These findings present an interesting comparison between the concepts of solubility and solubilization. If the monomers play a role as surface active agents, then this may invalidate the assumption that the aqueous phase is a uniform solution with water as the solvent. The actual distribution of various components in homogeneous systems can be difficult to deduce, though phase behaviour experiments have provided some insight. The high viscosity of the surfactants makes it relatively easy to identify the phase in which the surfactants are concentrated for multiphase samples, particularly since excess surfactant tends to form solid aggregates or highly concentrated "sponge" phases. Homogeneous samples have uniform viscosity, with optical characteristics that more closely resemble the oil and water than the surfactant blend, suggesting that the surfactants are well distributed. Phase behaviour studies with no monomer in the aqueous phase led to aggregation and precipitation of the surfactants as the surfactants came into contact with the water, even in the presence of silicone oil. That the presence of monomers, particularly HEMA, prevents this aggregation suggests that the monomers act to shield the water 
from the silicone surfactants. Taken into context with conductivity measurements, the implication is that at equilibrium, there remains a concentration gradient of conducting agents, HEMA and MAA within the aqueous phase. This potential orientation of aqueous phase components also helps to explain the suppressed conductance found at high aqueous phase volume fractions - even though the aqueous phase is continuous, the conducting agents are not necessarily uniformly distributed.

\subsection{THE COUNTER-ION EFFECT}

The nature of the counter-ion is expected to have a strong effect on ionic surfactants, reducing the ionic repulsion between the surfactant head groups. The effect is less clear with nonionic surfactants. It is believed that the introduction of a counter-ion may increase solubilization of the oil phase, an effect associated with the "salting out" of the surfactant ${ }^{4}$. According to the HLD framework, the change would be reflected in the salinity parameter, $b$. The value for sodium has been estimated at 0.13 , while the value for potassium is estimated at 0.09 , indicating that the smaller cation promotes a more positive shift in $\operatorname{HLD}^{4-6}$. At the salinity used to calculate HLD values at pH 6 in Table 2-3, the change from sodium to potassium would lower the HLD value by 0.6 . To add context, the width of the three-phase region for $3 \mathrm{cSt}$ silicone seen in Figure 2-2B corresponds to an HLD range of 2.2, and the temperature transition from 25 ${ }^{\circ} \mathrm{C}$ to $55^{\circ} \mathrm{C}$ corresponds to a positive shift of 1.8 . Thus we hypothesize that: 
If the sodium is replaced with a smaller cation, then the phase behaviour will shift to reflect a more positive HLD value, and if sodium is replaced with a larger cation, then phase behaviour will shift to reflect a more negative HLD value.

In order to evaluate the counter-ion effect on the silicone surfactant systems, the sodium hydroxide solution was replaced with lithium hydroxide, potassium hydroxide, or cesium hydroxide. Phase behaviour studies were conducted as described in section 2.2.5 for each formulation.

3.4.1 Phase behaviour variation with counter-ion size 


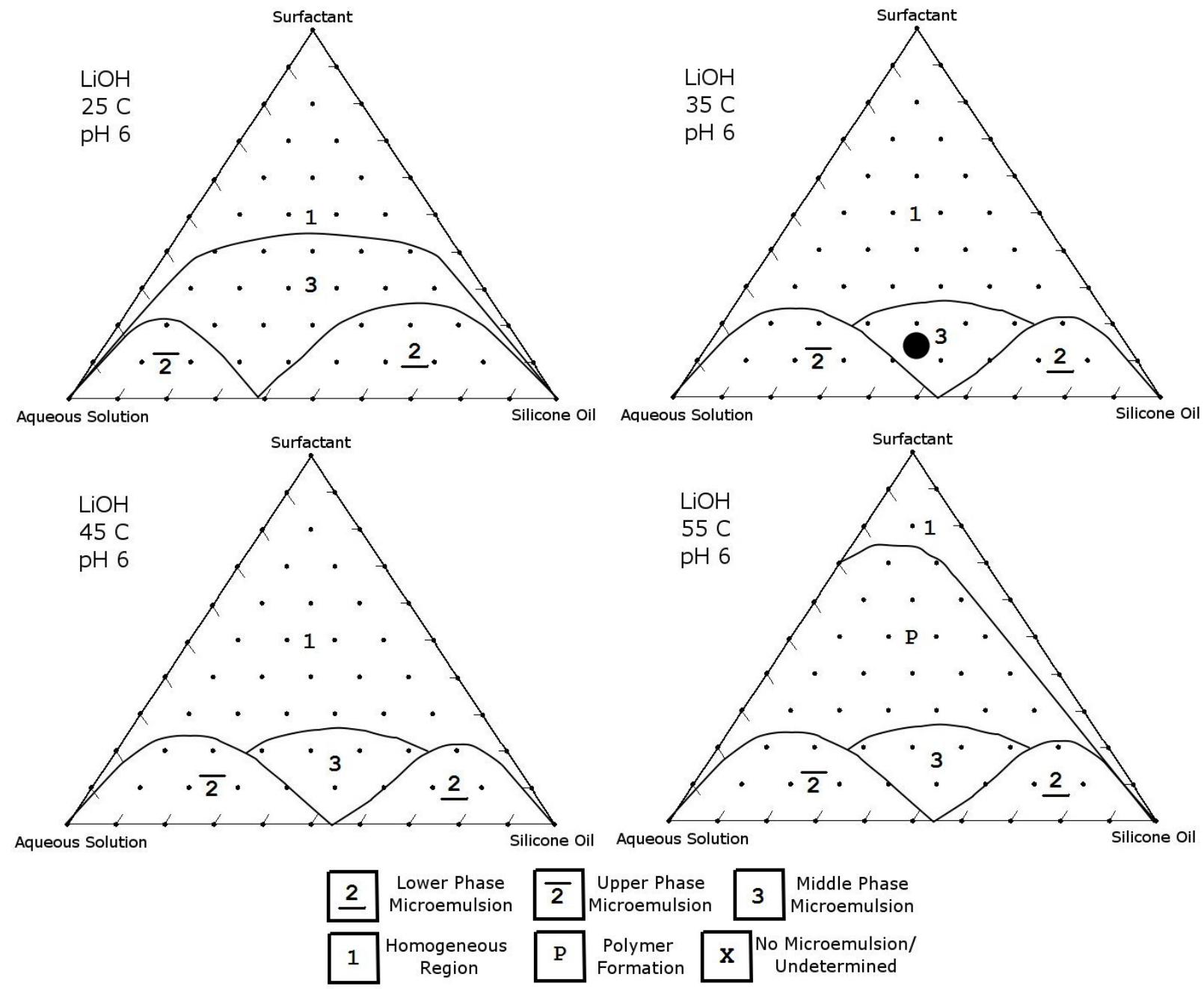

Figure 3-4: Phase behaviour for pH 6 systems adjusted with LiOH. Each point represents one sample (10\% increments by volume), with higher resolution ( $2 \%$ increments) near phase boundaries. Point of comparison at $35^{\circ} \mathrm{C}$ marked with black circle.

The phase behaviour for formulations containing $\mathrm{LiOH}$ is presented in Figure 3-4, with $\mathrm{KOH}$ presented in Figure 3-5 and $\mathrm{CsOH}$ in Figure 3-6. The HLD shift is evaluated qualitatively by comparing the relative size of regions with upper phase, middle phase and lower phase microemulsions. As solubility of Cesium at $25{ }^{\circ} \mathrm{C}$ is limited, the phase behaviour at $35{ }^{\circ} \mathrm{C}$ is used as the basis for comparison. The point of comparison is marked in each figure. 


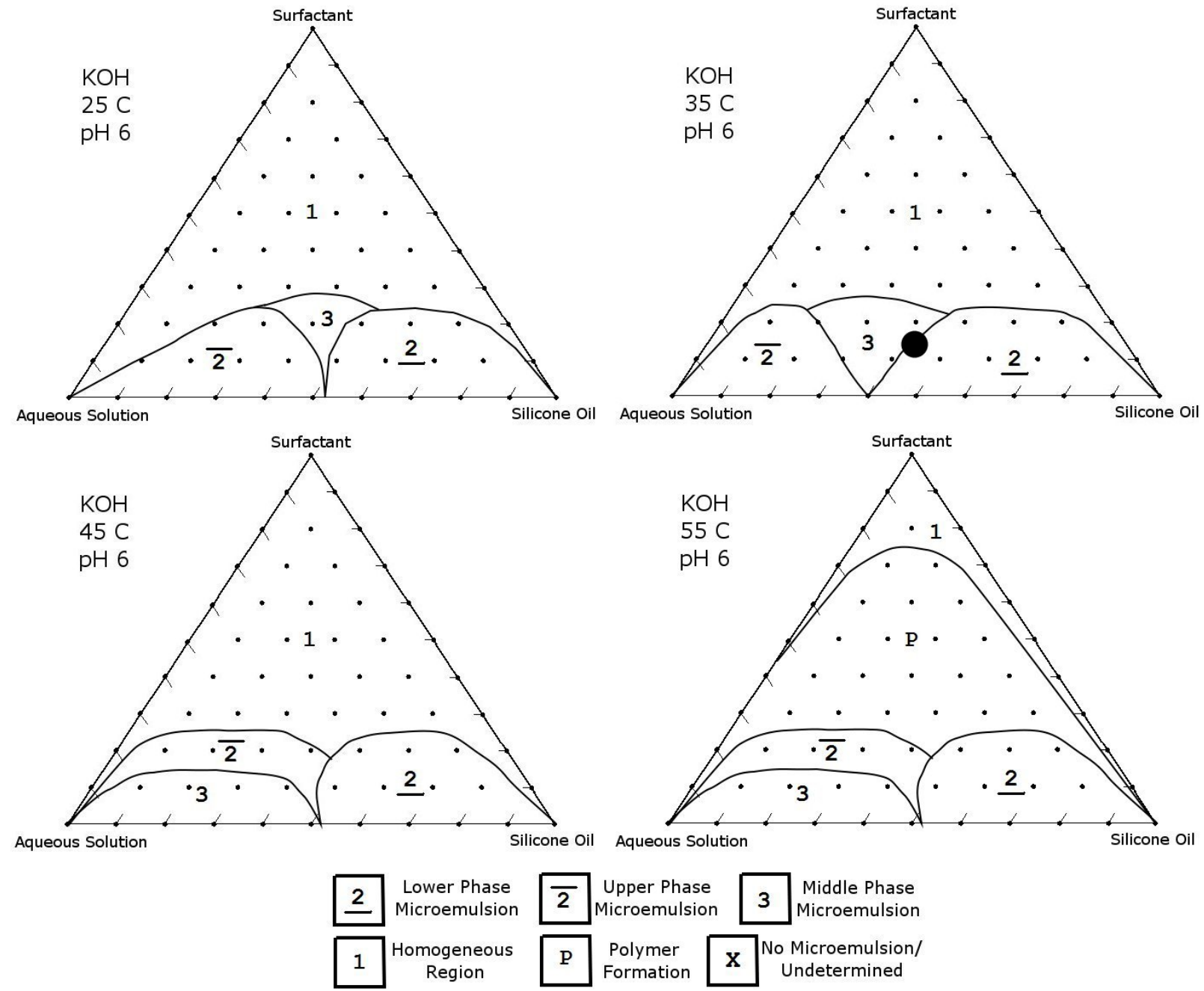

Figure 3-5: Phase behaviour for pH 6 systems adjusted with KOH $(\mathrm{n}=3)$. Each point represents one sample (10\% increments by volume), with higher resolution $(2 \%$ increments) near phase boundaries. Point of comparison at $35^{\circ} \mathrm{C}$ marked with black circle.

As with the $\mathrm{NaOH}$ formulation, the three formulations presented here were less temperature sensitive and less likely to polymerize spontaneously than the formulation at $\mathrm{pH} 2.5$, providing further supporting evidence for the hypothesis that increased MAA solubility would reduce polymerization and precipitation. This also indicates that the size of the cation does not play a 
significant role in MAA solubility. The pseudo-ternary phase diagrams indicate an increasing tendency towards larger areas of lower phase microemulsions as the cation size increases with $\mathrm{Li}^{+}<\mathrm{Na}^{+}<\mathrm{K}^{+}<\mathrm{Cs}^{+}$. This is evidenced both by the area covered in each ternary diagram by the respective microemulsion types, and by the phase behaviour around the marked point of comparison. In the $\mathrm{LiOH}$ formulation seen in Figure 3-4, this point is located within a three phase region near the middle $\rightarrow$ upper microemulsion boundary. In the $\mathrm{NaOH}$ formulation in Figure 2-7, this point remains in a three phase region, but near the middle $\rightarrow$ lower microemulsion boundary.

In the $\mathrm{KOH}$ formulation shown in Figure 3-5, the point of comparison straddles the middle $\rightarrow$ lower microemulsion boundary, and in the $\mathrm{CsOH}$ formulation in Figure 3-6, this point is within the lower phase microemulsion region. This behaviour is consistent with a shift to lower HLD values, corresponding to the decreasing value of $b$ predicted by Bourrel ${ }^{5}$. 


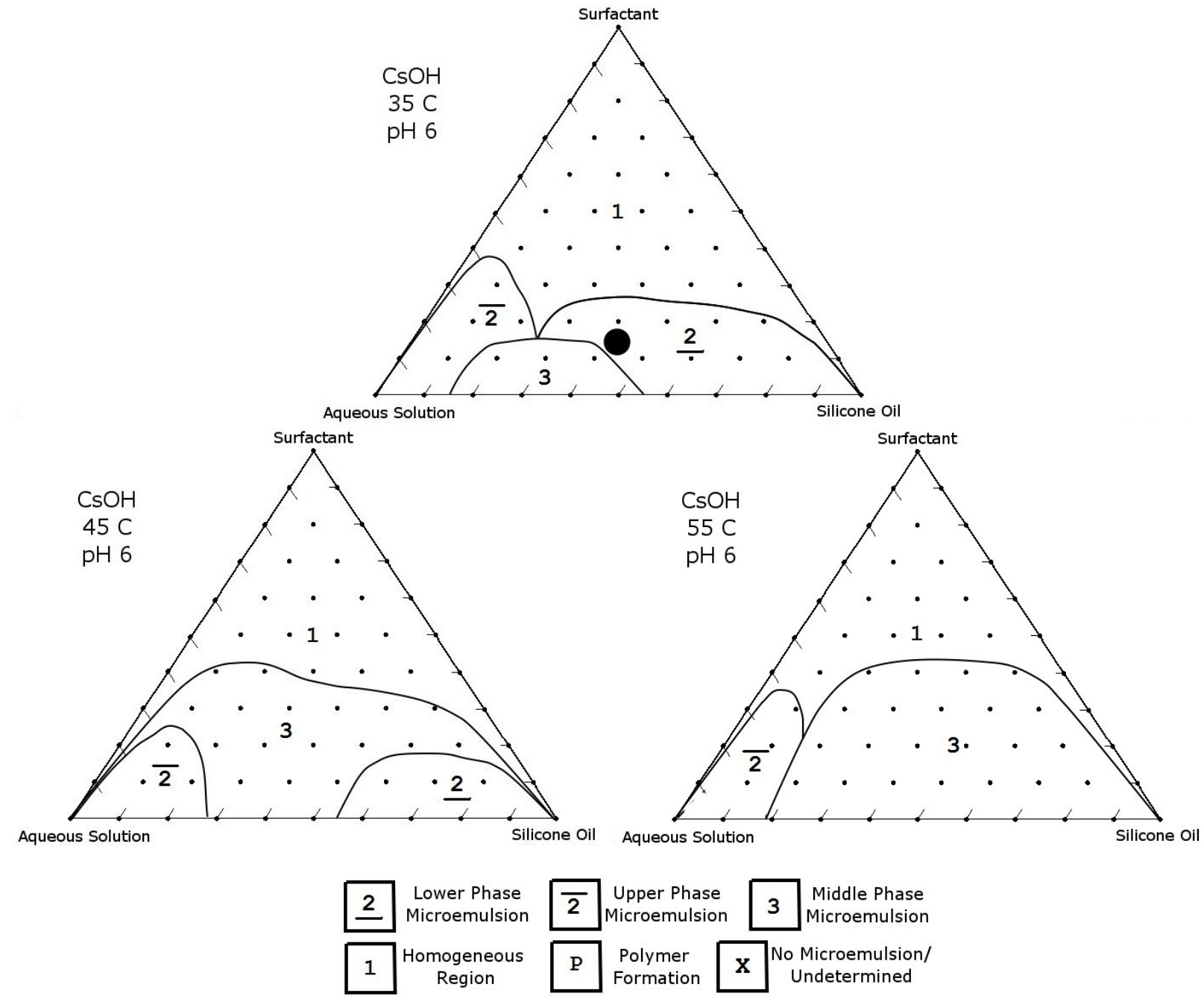

Figure 3-6: Phase behaviour for $\mathrm{pH} 6$ systems adjusted with $\mathrm{CsOH}(\mathrm{n}=3)$. Each point represents one sample (10\% increments by volume), with higher resolution $(2 \%$ increments) near phase boundaries. Cesium precipitation occurred at $25^{\circ} \mathrm{C}$, thus the phase behaviour reflects only the liquid phases. Point of comparison at $35^{\circ} \mathrm{C}$ marked with black circle.

\subsection{CONCLUDING REMARKS}

It has been suggested that the aqueous monomers, MAA and HEMA, play a role as surface active agents, and their effect as co-surfactants was quantified using a linear approximation for 
the co-surfactant function, $\varphi(\mathrm{A})$, as described by Nardello ${ }^{1}$. However, while this approach may be used to describe the role of the monomers in solubilization as co-surfactants, it does not sufficiently address their role as solutes. Additional phase behaviour and conductance studies were required to evaluate this behaviour. Results suggest that the aqueous phase may not be a uniform solution, but that components exist along a concentration gradient. This distinction may become important as the methodology for formulating polymerizable microemulsions is extended to other hydrophilic monomers, and it is likely that any hydrophilic monomer will require additional characterization beyond the HLD framework. An important consequence of these findings is the implication that the HLD equation does not adequately characterize the nature of the polar phase, and that it may be possible to develop the current co-surfactant terms into characteristic terms equivalent to the EACN for oils and $\mathrm{C}_{\mathrm{c}}$ for surfactants.

$\mathrm{NaOH}$ solution was incorporated into the microemulsion in order to raise the $\mathrm{pH}$, resulting in MAA dissociation and greater solubility. The effect of replacing sodium as the counter-ion was investigated, with results indicating that the increase in $\mathrm{pH}$ played a greater role on reducing temperature-sensitivity than the size of the cation. However, it has been demonstrated that the salting out effect of smaller cations causes a larger positive shift in the HLD values than for larger cations, which is consistent with the findings and estimates of the salinity parameter, $b$, reported by Bourrel ${ }^{5}$. 


\subsection{REFERENCES}

1. Nardello, V.; Chailloux, N.; Poprawski, J.; Salager, J. -L.; Aubry, J. -M. Polym. Int. 2003, 4, 602-609.

2. Salager, J.-L. Formulation Concepts for the Emulsion Maker. In Pharmaceutical Emulsions and Suspensions; Nielloud, F., Marti-Mestres, G., Eds.; MARCEL DEKKER, INC.: New York, 2000; pp 19.

3. Salager, J. -L.; Marquez, N.; Graciaa, A.; Lachaise, J. Langmuir 2000, 13, 5534-5539.

4. Rosen, M. J. Surfactants and Interfacial Phenomena, 3rd edition; John Wiley and Sons: New York, 2004; .

5. Bourrel, M.; Salager, J. L.; Schechter, R. S.; Wade, W. H. J. Colloid Interface Sci. 1980, 2, 451-461.

6. Kitahara, A. Journal of Physical Chemistry Journal of Physical Chemistry 1966, 11, 33943398. 


\section{INTERPENETRATING POLYMER NETWORKS TEMPLATED ON BICONTINUOUS MICROEMULSIONS CONTAINING SILICONE OIL, METHACRYLIC ACID AND HYDROXYETHYL METHACRYLATE}

\subsection{INTRODUCTION AND BACKGROUND}

Conventional interpenetrating polymer networks (IPN) are formed from two or more crosslinked polymer networks that are physically entangled but not covalently linked. The resultant properties reflect those of the original components to a greater degree than synthesis by copolymerization. Though each component may not necessarily be continuous, this structure allows for the networks to be combined on a range of scales, with the resulting material having some combination of chemical and physical properties of the original polymers ${ }^{1}$. For example, this technique can apply the mechanical strength, hydrophilicity/hydrophobicity or lubricity of one component to support a second component. IPNs can be formed through sequential or simultaneous polymerization. Sequential polymerization is primarily used to combine materials requiring incompatible polymerization environments or techniques. The "host" polymer is prepared first, and is then swollen with "guest" monomers and crosslinkers. The result is that the host polymer is likely to form the continuous phase, and the structure of the guest monomer is constrained by the high viscosity polymerization environment. Simultaneous polymerization, where multiple sets of monomers, crosslinkers and activating agents are pre-mixed, is limited to formulations with non-interfering reactions ${ }^{1,2}$. The morphology of the IPN is dependent on 
several key factors, such as the compatibility of the polymers and polymerization reactions, the scale of mixing, and the kinetics of each reaction. Compatibility issues such as co-solubility and relative hydrophobicity between the polymers are major constraints in IPN formation, as well as the complexity of the synthesis. These issues are particularly evident when trying to polymerize networks of hydrophobic and hydrophilic polymers simultaneously ${ }^{3-7}$.

IPNs of silicone rubbers and hydrogels are of particular interest as biomaterials, as they combine the elasticity and gas transport properties of a silicone phase with the tunable aqueous transport properties of a hydrogel ${ }^{2}$. The IPN is commonly formed via sequential polymerization, with the silicone rubber acting as the host material. PDMS-PHEMA networks reported by Abbasi et al. ${ }^{8}$ and PDMS-PVA networks reported by Pavlyuchenko et al. ${ }^{9}$ were formed in this way. This method also allows for the inclusion of functional and responsive hydrogel elements. For example, PDMS-PNIPAAM IPNs were formulated by Liu et al. ${ }^{10}$ to take advantage of the reversible LCST phase transition. Similarly, PDMS-PMAA IPNs with bicontinuous morphologies and up to $30 \%$ PMAA on a dry mass basis were formulated by Turner et al. ${ }^{11,12}$, in order to exploit the $\mathrm{pH}$-responsiveness of PMAA. The desired morphologies are achieved by controlling the chemical potential of the guest monomer in the PDMS pre-IPN film.

An interesting alternative to the sequential methods presented above is the use of bicontinuous microemulsions as low viscosity templates for the desired IPN morphology. Microemulsions are nanostructured systems containing oil and water domains stabilized by surfactant. The structures are typically identified as oil in water (micelles), water in oil (reverse micelles) or bicontinuous. 
In a bicontinuous microemulsion, both phases are continuous and interpenetrating. The nanostructure is dependent on phase behavior, which in turn can be controlled by varying composition and temperature ${ }^{13-16}$. Most importantly, this controllable nanostructure is readily exploited as a platform for chemical reactions, including polymerization ${ }^{17-19}$. Each phase domain essentially acts as a self-contained vessel, providing distinct reaction environments at the nanoscale. Chow and Gan ${ }^{20}$ have a provided an extensive review of techniques for controlling microemulsion phase behavior to generate reaction templates and polymers. Several groups have also investigated polymerization of hydrocarbon monomers. However, studies to date have generally focused on polymerization in the aqueous phase. ${ }^{20-25}$

While microemulsion templates theoretically allow for controllable domain size and structure with the potential for higher surface area to volume ratios than traditional polymerization methods, practical limitations to the polymerization process have thus far restricted the range of structures that can be produced. Most importantly, the polymerization itself disrupts the thermodynamic equilibrium that led to the original template ${ }^{26,27}$. There are several possible approaches to the problem of shifting equilibrium structures and polymerization time scales, with current efforts focused on stabilizing the original structure during polymerization by immobilizing one or both phases, analogous to sequential polymerization. In order to proceed with simultaneous polymerization, the structure should be quenched faster than the rate at which the microemulsion re-equilibrates in response to shifting conditions. Polymerizable surfactants can strengthen and preserve the interfacial layers in micellar microemulsions, as demonstrated by Summers et al. ${ }^{28}$. In polymerization attempts by Gao ${ }^{29}$, the aqueous phase consisted of a concentrated sugar solution, resulting in bicontinuous domains of monomer and glassy sugar 
that yielded a near 1:1 replica of the template when polymerized. Stubenrauch et al. ${ }^{30,31}$ and Magno et al. ${ }^{32}$ have stabilized a bicontinuous microstructure containing an Nisopropylacrylamide (NIPAAM) loaded aqueous phase by forming an organogel from the oil phase as a template/scaffold. These techniques have proven effective in preserving microemulsion structure, but have yet to be extended to simultaneous polymerization of a bicontinuous system.

In this study, we investigate the simultaneous polymerization of PDMS/P(MAA-HEMA) IPNs templated on bicontinuous microemulsion microstructure with and without incorporating polymerizable silicone surfactants. As described in chapter 1, this includes the proposition that the classical IPN definition be extended to included physically entangled networks on a larger scale. We have reported the phase behavior of microemulsions containing silicone oil, silicone alkyl polyether surfactants, and an aqueous solution of MAA and HEMA ${ }^{33}$, and this work is summarized in chapters 2 and 3. By characterizing the effects of each component on the phase behavior, we are able to identify compositional ranges that are suitable as potential polymerization templates. In the current work, microemulsions with the desired morphology are first prepared using low viscosity prepolymer and monomer solutions, and are then polymerized and crosslinked simultaneously. The process is intended to provide a simpler approach to forming IPNs with interpenetration at the near molecular level, and from a wide range of monomers. In addition to the primary hypothesis, we investigate the secondary hypothesis that: 


\section{If polymerizable surfactants replace non-reactive surfactants in formulation, the resulting IPN will retain smaller domains}

The polymers produced are characterized by laser scanning confocal microscopy (LCSM), differential scanning calorimetry (DSC), swelling behavior and permeability.

\subsection{EXPERIMENTAL}

\subsubsection{Materials}

MAA (99\%), HEMA (97\%) and triethylene glycol dimethacrylate (97\%) were obtained from Sigma Aldrich and purified by vacuum distillation. Vitamin B12 and fluorescein (sodium salt) was also obtained from Sigma Aldrich, and used as provided. A 10N sodium hydroxide solution was purchased from VWR Canada. A water soluble, radical AZO initiator 2,2'-Azobis[2-(2imidazolin-2-yl)propane] dihydrochloride (VA-044) was purchased from Wako Specialty Chemicals, with a 10 hour half life decomposition temperature of $\mathrm{T} 1 / 2,10=44{ }^{\circ} \mathrm{C}$ in aqueous solution, which is amongst the lowest commercially available. Hydride and vinyl terminated 3 centistoke (cSt) silicone oils, methylhydrosiloxane-dimethylsiloxane copolymer (crosslinking agent) and a platinum divinyl-tetramethyldisiloxane catalyst were obtained from Gelest. Silicone lauryl polyether surfactants (Silube J208-612, 812) and silicone acrylate surfactants (Silmer ACR D208, Di-1508) were donated by Siltech Corporation. Properties of the surfactant lines are presented in Table 4-1: Summary of silicone surfactant properties ${ }^{34}$. The generic structure for the Silube series is shown in Figure 2-1: General structure of silicone alkyl 
polyether surfactant (PEG-8 Dimethicone). The hydrophilicity is determined by the ratio of polyether to silicone and alkyl groups. 46. The generic structures for the Silmer series are shown in Figure 4-1.

Table 4-1: Summary of silicone surfactant properties ${ }^{34}$

\begin{tabular}{|c|c|c|c|c|}
\hline Surfactant & $\begin{array}{l}\text { Product } \\
\text { Designation } \\
\end{array}$ & $\begin{array}{l}\text { Viscosity (cps) at } \\
25^{\circ} \mathrm{C} \\
\end{array}$ & Functionality & IPN Designation \\
\hline A & Silube J208-812 & 300 & None & $\mathrm{NR}-\mathrm{IPN}^{\mathrm{b}}$ \\
\hline B & Silube J208-612 & 600 & None & \\
\hline $\mathrm{C}$ & $\begin{array}{l}\text { Silmer ACR Di- } \\
1508\end{array}$ & $750-2500$ & $\begin{array}{l}\text { Acrylate, } \\
\text { Difunctional }\end{array}$ & $\mathrm{C}-\mathrm{IPN}$ \\
\hline $\mathrm{D}$ & Silmer ACR D208 & $300-1000$ & $\begin{array}{l}\text { Acrylate, } \\
\text { Multifunctional }\end{array}$ & $\mathrm{D}-\mathrm{IPN}$ \\
\hline
\end{tabular}




$$
\begin{aligned}
& \mathrm{CH}_{3} \quad \mathrm{CH}_{3} \quad \mathrm{CH}_{3} \\
& \mathrm{R}-\left(\mathrm{CH}_{2}\right)_{3}-\mathrm{Si}-(\mathrm{O}-\mathrm{Si})_{\mathrm{a}}-\mathrm{O}-\mathrm{Si}-\left(\mathrm{CH}_{2}\right)_{3}-\mathrm{R} \\
& \begin{array}{llll}
\mathrm{CH}_{3} & \mathrm{CH}_{3} & \mathrm{CH}_{3} & \text { Surfactant } \mathrm{C}
\end{array} \\
& \mathrm{R}=\left(\mathrm{OCH}_{2}-\mathrm{CH}_{2}\right)_{\mathrm{c}}-\left(\mathrm{OCH}_{2}-\mathrm{CH}\right)_{\mathrm{d}}-\mathrm{OCCH}=\mathrm{CH}_{2} \\
& \mathrm{CH}_{3} \quad \mathrm{O} \\
& \begin{array}{cccc}
\mathrm{CH}_{3} & \mathrm{CH}_{3} & \mathrm{CH}_{3} & \mathrm{CH}_{3}
\end{array}
\end{aligned}
$$

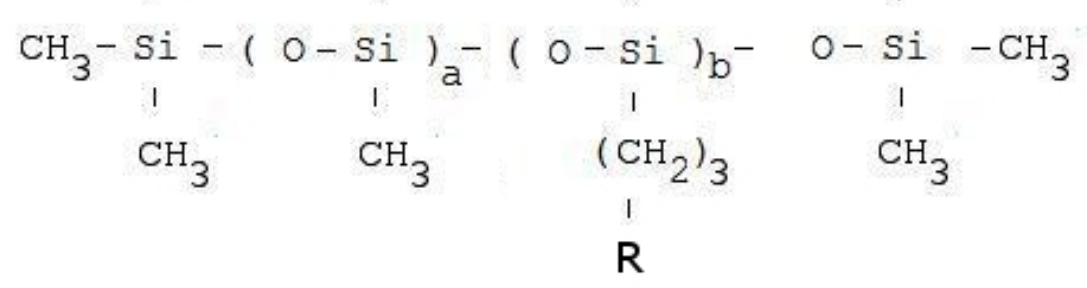

Figure 4-1: Generic structures for the acrylate-functional Silmer series. ${ }^{34}$

\subsubsection{Microemulsion preparation}

An aqueous solution at $\mathrm{pH} 6$ was prepared from MAA, HEMA, and sodium hydroxide solution diluted with deionized water, at a ratio of 2:3:4 by volume (1:1:9.4 molar). The oil phase was prepared with equal parts hydride and vinyl terminated $3 \mathrm{cSt}$ silicone oil (prepolymer, 1:1 mol) and $10 \% \mathrm{v} / \mathrm{v}$ methylhydrosiloxane-dimethylsiloxane copolymer $(3 \% \mathrm{~mol} / \mathrm{mol})$. For studies with non-reactive surfactants, the surfactant blend used consists of a 50/50 mixture of surfactants A 
and B by volume (1.1:1 molar). For studies with reactive surfactants, the surfactant component consists of $100 \%$ surfactant C or D. The systems studied here take advantage of the linker approach to microemulsion formulation, using mixtures of hydrophilic and lipophilic components to form a series of mediating connections to enhance the solubilization of one phase into the other ${ }^{35-37}$. As the silicone alkyl polyether surfactants are not miscible with water on their own, the MAA and HEMA act as co-solvents that mediate the formation of a microemulsion. $^{33}$

\subsubsection{Phase behavior studies}

The phase behavior of potentially polymerizable formulations was investigated over a range of temperatures using ternary phase diagrams in order to identify compositional regions that remain isotropic and homogeneous above the $\mathrm{T}_{1 / 2,10}$ of the Azo initiator. Ternary phase diagrams were populated with 66 samples containing varying compositions of aqueous solution, reactive oil blend and surfactant, with compositional changes in $10 \%$ increments by volume between adjacent samples, as previously described ${ }^{33}$. When adjacent compositions showed a change in phase behavior, additional samples were added in 5\% increments to more precisely resolve the phase boundary. Mixtures were characterized by the number of isotropic liquid phases and microemulsion type, if any. To determine the effect of temperature on phase behavior, samples were placed in a water bath with a Thermo Electron Corporation Haake DC 30 circulator and allowed to equilibrate for 8 hours or longer, until they appeared to be visibly stable. Phase transitions were initially identified by visual observation, and confirmed by conductance measurements on formulations that formed microemulsions. 
The presence of liquid crystals in homogeneous microemulsions was assessed for samples with greater than $70 \%$ surfactant by volume and samples along the aqueous-surfactant axis of the ternary phase diagram. $500 \mu \mathrm{L}$ of each sample was deposited onto glass microscope slides with glass cover slips, and was examined under the cross-polarizing filter for birefringence.

Bicontinuity in homogeneous microemulsions was assessed by comparing its conductance to that of the aqueous phase. Conductance, indicative of aqueous phase continuity, was measured at $25^{\circ} \mathrm{C}$ using a VWR/Traceable portable conductivity meter with a glass probe (cell constant of 1) supplied by Microelectrodes, Incorporated. Measurements were taken by inserting the exposed platinum probes to the middle of a $2 \mathrm{~mL}$ homogeneous microemulsion. Oil continuous microemulsions are expected to have a near-zero normalized conductance (sample conductance/aqueous phase conductance), while the normalized conductance of bicontinuous samples should range from 0 to $1 .^{33,38-41}$

\subsubsection{Microemulsion polymerization}

For polymerization experiments, $1 \% \mathrm{w} / \mathrm{v}$ (monomer) Azo initiator and 1 to $10 \% \mathrm{v} / \mathrm{v}(0.7-7 \%$ $\mathrm{mol} / \mathrm{mol}$ ) triethylene glycol dimethacrylate (TEGDMA) was incorporated into the aqueous solution. The TEDGMA content reported for formulations in this paper is based on the total amount of MAA, HEMA and TEDGMA in solution. Surfactant, reactive oil blend and aqueous 
solution were combined in a 3:2:5 ratio by volume based on results from phase behavior studies. This yields an aqueous monomer (HEMA and MAA) to silicone prepolymer ratio of 1.38:1 by initial volume or $1.57: 1$ by initial mass. Samples were vortexed for 60 seconds, and were then allowed to equilibrate for 24 hours at $20^{\circ} \mathrm{C}$. As the platinum catalyst facilitates PDMS crosslinking at room temperature, its addition is delayed in order to prevent PDMS curing during initial equilibration. Prior to polymerization, $1 \% \mathrm{v} / \mathrm{v}(1.3 \% \mathrm{~mol} / \mathrm{mol}$, silicone phase $)$ platinum catalyst was gently mixed into the microemulsion, which was allowed to re-equilibrate for 15 minutes. $5 \mathrm{~mL}$ of the resulting microemulsion were spread into a $60 \mathrm{~mm}$ diameter glass petri dish. Polymerization was carried out by heating the microemulsion to $50{ }^{\circ} \mathrm{C}$ for 3 hours in a sealed oven purged with $\mathrm{N}_{2}$. The resulting material was washed extensively in acetone and distilled water in order to remove the surfactant, excess monomer and excess oligomer. Control P(MAA-HEMA) hydrogels for swelling comparison were also prepared from the aqueous solution described above by heating samples for 3 hours at $50{ }^{\circ} \mathrm{C}$ in an $\mathrm{N}_{2}$ atmosphere. Similarly, control PDMS samples were prepared by curing the silicone phase described above for 3 hours at $50^{\circ} \mathrm{C}$.

\subsubsection{Swelling Behavior and Permeation}

Samples for swelling studies were removed with a $4 \mathrm{~mm}$ biopsy punch and were placed in distilled water for 2 weeks. The samples were characterized by hydration, defined as the mass fraction of water in the swollen IPN (water mass/total swollen mass), with water mass calculated as the difference between total swollen and total dry mass. 
Permeation studies were conducted at $20{ }^{\circ} \mathrm{C}$ using a standard two-compartment diffusion cell and IPN membranes pre-equilibrated in distilled water. An aqueous solution of vitamin $\mathrm{B}_{12}$ in distilled water $\left(\mathrm{C}_{\text {donor }}=60 \mu \mathrm{g} / \mathrm{mL}\right)$ was loaded into the donor chamber. The receptor chamber was initially filled with an equal volume of distilled water, and was sampled at 4 hour intervals. The samples were tested in a Hewlett Packard 8452a UV-Visible spectrophotometer for the presence of $B_{12}$ at $362 \mathrm{~nm}$. Approximate infinite sink conditions were maintained by replacing the contents of the donor and receptor chamber with fresh $\mathrm{B}_{12}$ solution and distilled water, respectively, after each sampling. Membrane permeability to vitamin $\mathrm{B}_{12}$ was calculated from the average steady state flux, exposed cross-sectional area and swollen membrane thickness.

\subsubsection{Laser Scanning Confocal Microscopy (LSCM) and Differential Scanning Calorimetry (DSC)}

While IPN domains are generally much smaller than can normally be resolved by LSCM, we employ the technique previously described by Turner et al. to image hydrogel domains in the

swollen state ${ }^{11}$. LSCM allows for observation of continuous aqueous channels by highlighting the penetration of fluorescein through the IPN. Samples were removed with a $4 \mathrm{~mm}$ biopsy punch and were soaked in aqueous sodium fluorescein solution for 2 weeks. The fluorescein highlights accessible aqueous pathways, while PDMS domains and any excess surfactant remain dark. Imaging was conducted using an inverted Olympus Fluoview 300 with $488 \mathrm{~nm}$ excitation, $25 \%$ laser power, FITC filter cube and a 1.4 NA oil immersion objective. Images were captured at $512 \times 512$ resolution in 8-bit gray scale. Images were taken in $0.5 \mu \mathrm{m}$ depth intervals. Domain 
sizes were calculated using the particle analyzer tools in the ImageJ picture editing software package, and were based on a comparison of highlighted and dark regions.

DSC measurements were taken using a TA Instruments DSC 2010. $15 \mathrm{mg}$ samples of dried IPN, control P(MAA-HEMA) and control PDMS were loaded into aluminum hermetic pans, and were heated at $2{ }^{\circ} \mathrm{C} /$ minute. Heat flow differential was measured against an empty aluminum hermetic pan.

\subsubsection{Texture Analysis}

The textures of sample materials were analyzed in a Stable Micro Systems TA.XTplus texture analyzer with a cylindrical stainless steel probe. Samples were hydrated to equilibrium swelling. The probe was placed against the IPN surface, and was lowered to a depth of $5 \mathrm{~mm}$ at a rate of $0.5 \mathrm{~mm} / \mathrm{s}$. The force required to compress and penetrate the IPN was recorded as a function of time and depth, and was compared to control measurements on PDMS and hydrated P(MAAHEMA). 


\subsection{RESULTS AND DISCUSSION}

\subsubsection{Phase Behavior of Reactive Silicone Microemulsions}

The phase behavior of silicone microemulsions containing MAA and HEMA in aqueous solution, vinyl and hydride terminated $3 \mathrm{cSt}$ silicone oils and silicone-based surfactants was evaluated using pseudo-ternary phase diagrams evaluated at different temperatures. Diagrams for formulations with non-reactive silicone lauryl polyether surfactants are shown in Figure 4-2, and results for formulations with polymerizable silicone acrylate surfactants are shown in Figure 4-3.

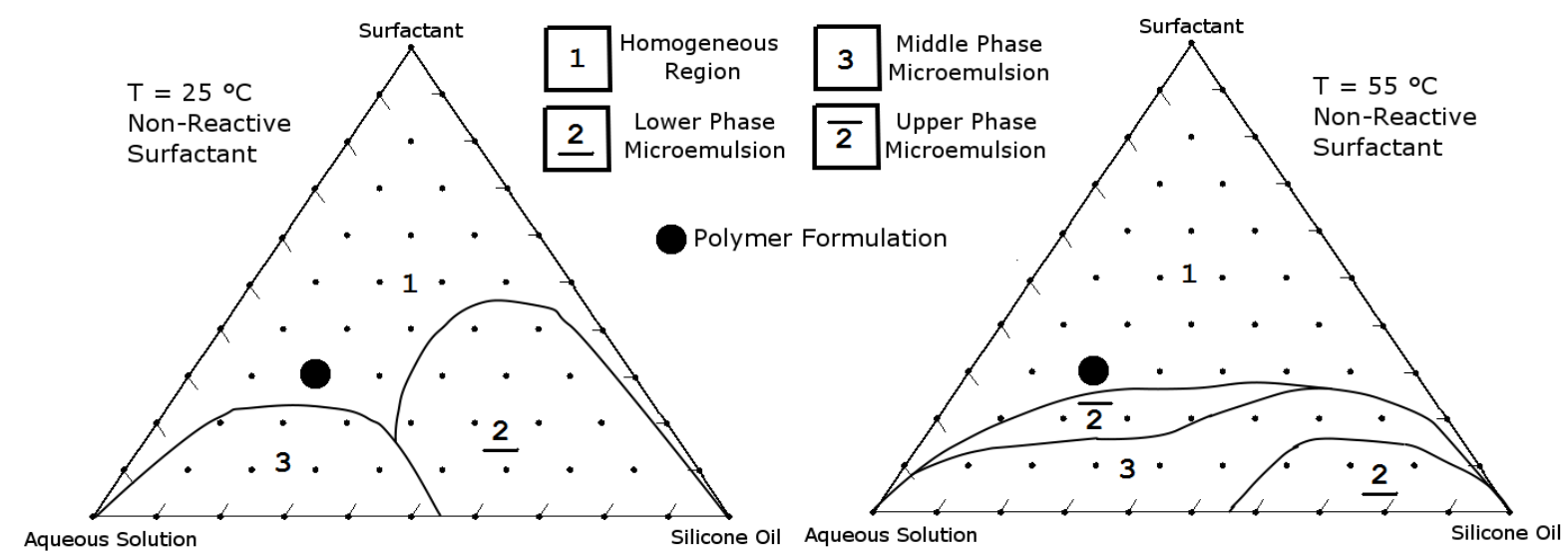

Figure 4-2: Phase behavior for formulations containing MAA and HEMA in aqueous solution at pH 6, vinyl and hydride terminated $3 \mathrm{cSt}$ silicone oil, and a blend of nonreactive silicone lauryl polyether surfactants at $\mathrm{T}=25^{\circ} \mathrm{C}$ (left) and $\mathrm{T}=55^{\circ} \mathrm{C}$ (right). Each point represents one sample $(10 \%$ increments by volume) with higher resolution $(5 \%$ increments, not marked) near phase boundaries. 


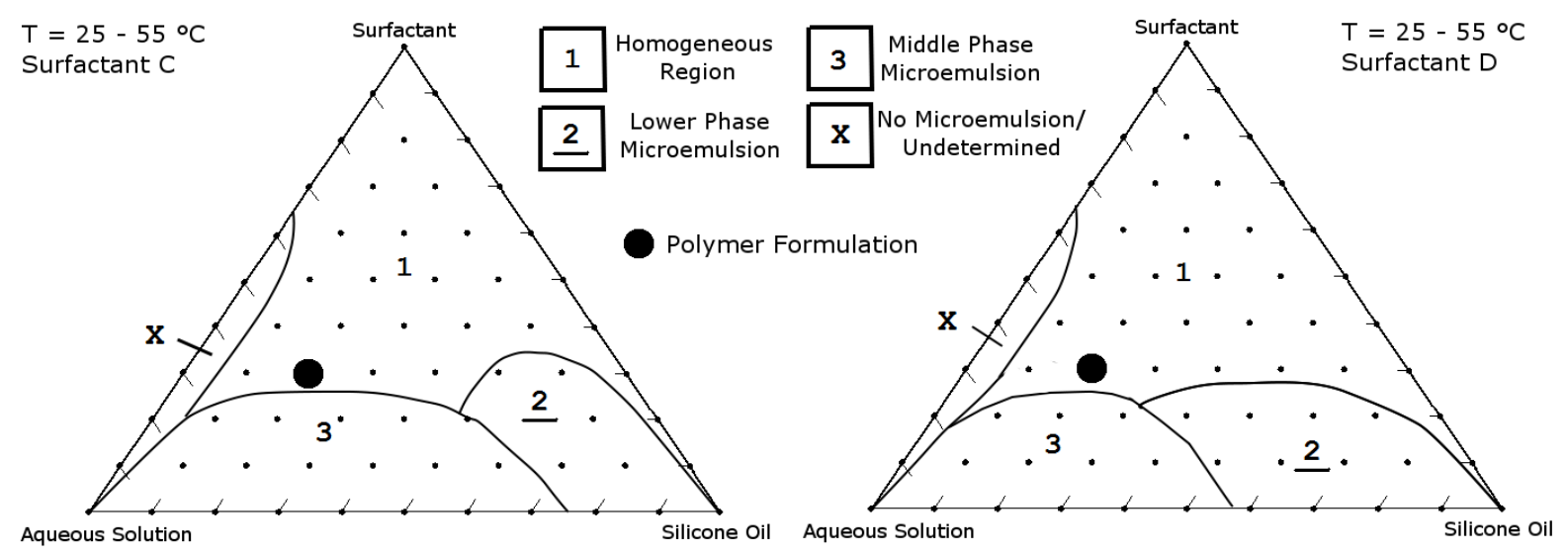

Figure 4-3: Phase behavior of formulations containing MAA and HEMA in aqueous solution at pH 6, vinyl and hydride terminated $3 \mathrm{cSt}$ silicone oil, and silicone acrylate surfactant $C$ (left) or $D$ (right) at $T=25,35,45$ and $55^{\circ} \mathrm{C}$. Phase behavior was observed to be unchanged over the range of temperatures investigated. Each point represents one sample (10\% increments by volume), with higher resolution (5\% increments, not marked) near phase boundaries.

When compared to ternary diagrams for non-reactive systems previously studied ${ }^{33}$, much of the expected phase behavior was conserved. The phase behavior above $25 \%$ surfactant content of all three formulations presented here was stable. In addition, no liquid crystal states were detected. The larger regions of 3-phase and lower phase microemulsions and the limited formation of upper phase microemulsions may indicate that the hydride and vinyl terminated silicone oils are more hydrophobic than their non-reactive, trimethylsiloxy terminated counterpart ${ }^{33}$. However, there remains a large, isotropic region from which to choose potentially polymerizable formulations. In comparing the non-reactive and reactive surfactants, it is interesting to note that phase behaviour for reactive surfactants did not change over the temperature range studied, while phase behaviour for non-reactive surfactants changed significantly. This would indicate that between $10 \%$ and $20 \%$ surfactant, the three phase region for reactive surfactants is wider than for non-reactive surfactants with respect to temperature. It may also indicate that the resolution was not small enough to detect slight changes in the phase boundaries. 
In order to minimize the amount of surfactant in the formulation and to allow for comparison between reactive and non-reactive surfactants, a ratio of 3:2:5 of surfactant to oil to aqueous solution by volume was selected for presentation and further study here. This polymer formulation is highlighted in Figure 4-2 and Figure 4-3. This selection also satisfies the requirement that the formulation remain isotropic and homogeneous over the temperature range studied for all three surfactant combinations. An upper bound for the persistence length, $\xi \leq 36$ nm was calculated for this formulation using the methodology developed by De Gennes et al. ${ }^{42}$ and expanded by Sottmann et al. ${ }^{43}$ for bicontinuous microemulsions. In this approach, the persistence length is calculated as:

\section{4-1: $\xi=6\left(\varphi_{\text {oil }} \varphi_{\text {aqueous }}\right) C_{s}^{-1} A_{s}^{-1}$}

where $\varphi_{\text {oil }}$ and $\varphi_{\text {aqueous }}$ are the volume fractions of the oil and aqueous phase, $\mathrm{C}_{\mathrm{s}}$ is the surfactant concentration, and $A_{s}$ is the area per surfactant molecule. The effective area per molecule, $A_{s}$ was estimated to be approximately $41 \AA^{2}$ using the Gibbs Adsorption equation and surface tension measurement ${ }^{44-47}$. As surfactants $\mathrm{A}$ and $\mathrm{B}$ are not water soluble, the next most hydrophilic surfactant in the series (surfactant Y, J208-412) was used in the estimate. The persistence length provides a basis for comparing the IPN morphology to the theoretical microemulsion morphology. In order to make this comparison, we must first estimate the IPN domain. 


\subsubsection{Microemulsion Polymerization and LSCM}

PDMS-P(MAA-HEMA) IPNs were prepared from bicontinuous microemulsion templates stabilized by non-reactive silicone alkyl polyether (NR-IPN) or reactive silicone acrylate (CIPN, D-IPN) surfactants. The yield based on polymerizable material was $90 \% \pm 4 \%$ for formulations with non-reactive surfactants, and $96 \% \pm 2 \%$ for those with reactive surfactants. As the yield is calculated based on final dry mass, it may include entrapped surfactant, excess monomer and untangled oligomer that were not removed during the washing steps. Hydrated IPNs at 5\% v/v TEGDMA are shown in Figure 4-4.

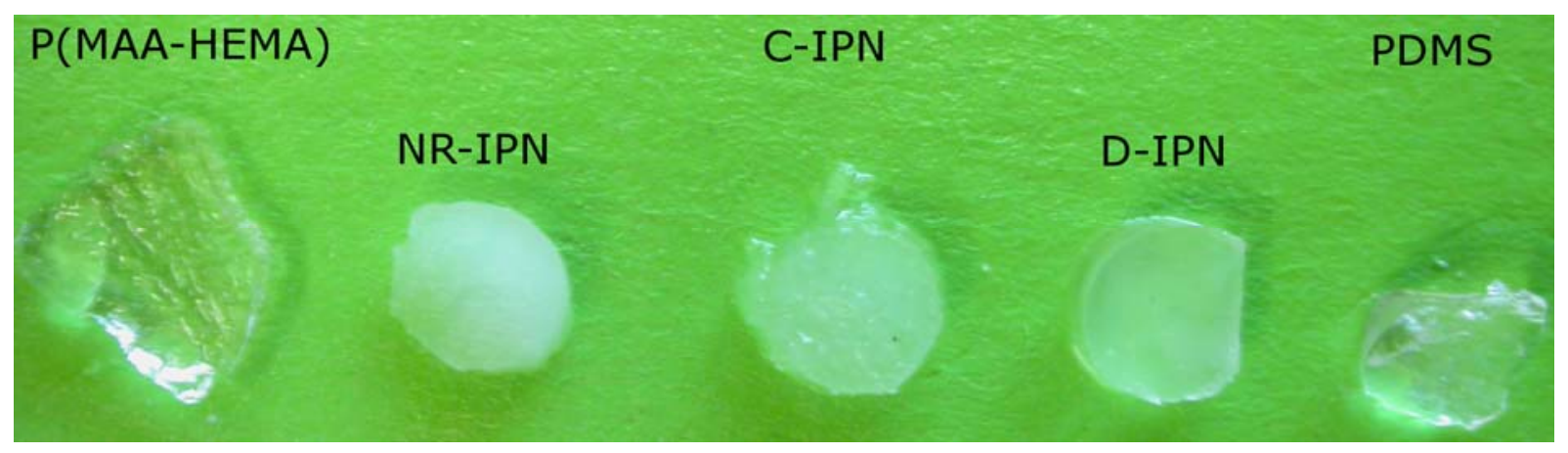

Figure 4-4: Comparison of hydrated IPNs at 5\% v/v TEGDMA with hydrated P(MAAHEMA) and PDMS components used in their formulation.

Despite the mixing scale and sub-micron domains in the bulk, the IPNs ranged from cloudy (NR-IPNs) to translucent (C-IPN, D-IPN) instead of remaining transparent, as seen in Figure 4-4. While the cloudiness may be indicative of a change in phase behavior affecting domain size, it can partially be attributed to the interaction between the excess surfactant and excess 
water. The silicone alkyl polyether surfactants form a high viscosity, turbid, white dispersion when mixed with water. Washing the polymer is intended to remove as much of this viscous phase as possible, in addition to excess monomer and untangled oligomer. However, the oily feel and cloudiness of the dried IPNs would suggest that some entrapped surfactant-water domains remain, reducing IPN transparency.

LSCM on samples swollen in aqueous sodium fluorescein was used to observe the hydrated polymer nanostructure. Images at equilibrium swelling for NR-IPNs at 5\% v/v TEGMDA are shown in Figure 4-5, with the top left image showing the surface that contacted the glass during polymerization. The highlighted aqueous channels suggest that both the oil and the aqueous domains are uniformly distributed, with domain size decreasing to $\sim 300 \mathrm{~nm}$ at a depth of $20 \mu \mathrm{m}$, as shown in Figure 4-6. Near the surface, there are large, irregular, unhydrated domains which could indicate pockets of PDMS, excess surfactant, distortion and damage due to sample preparation, and the surface effects at the glass-microemulsion interface during polymerization. Hydrogel domains encapsulated by PDMS remain inaccessible to the fluorescein and appear as dark spots. 3-D image reconstruction suggests that the dark domains are not spherical, as their dimensions are irregular. However, while this imaging supports connectivity in the aqueous channels, it does not confirm connectivity in the PDMS. 


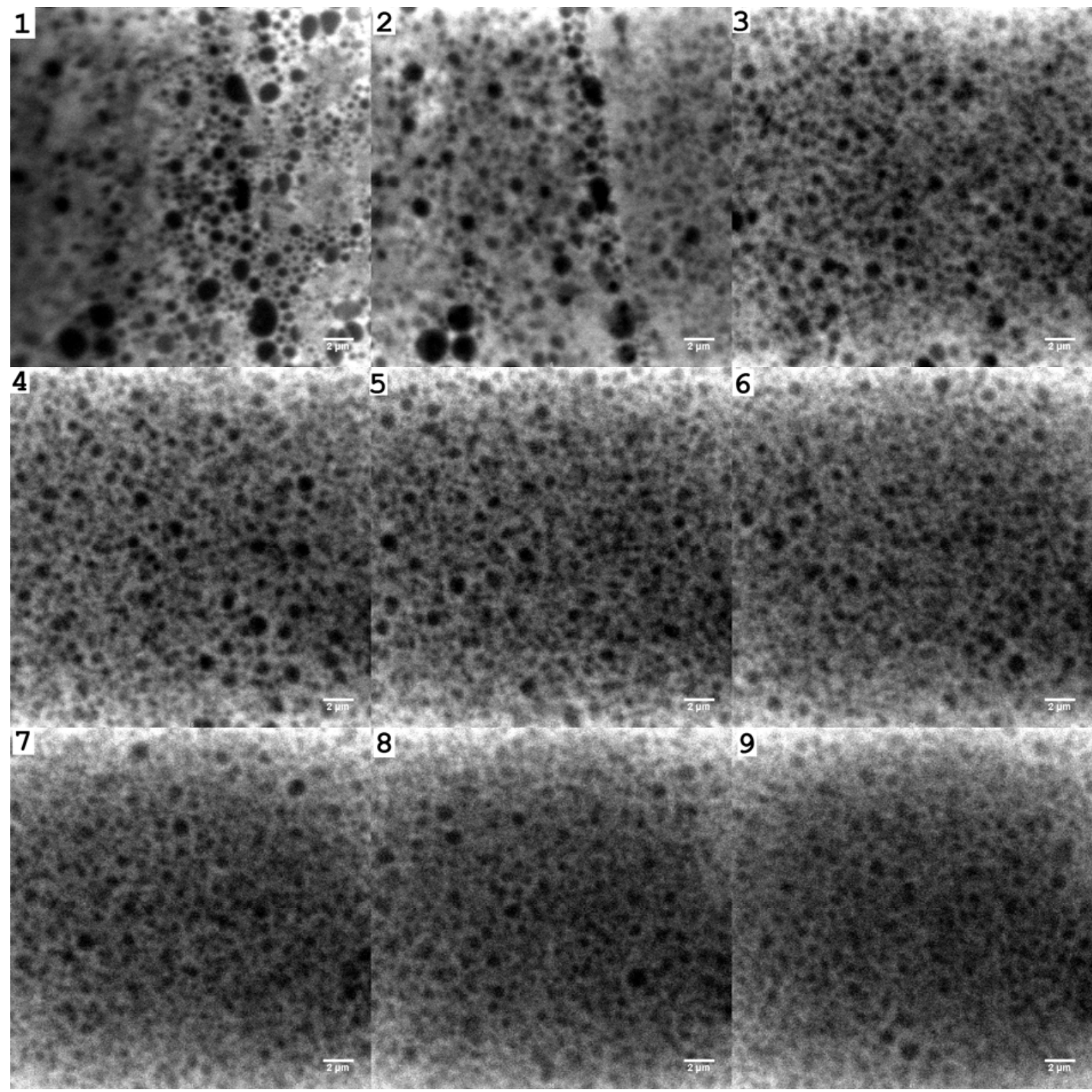

Figure 4-5: Selected LSCM images of NR-IPN at equilibrium swelling, 5\% v/v TEGDMA. Images proceed from left to right and top to bottom in $2.5 \mu \mathrm{m}$ increments. The top left image represents the glass-contacting surface, and the bottom right image represents a depth of $20 \mu \mathrm{m}$. Scale bars are $2 \mu \mathrm{m}$. 


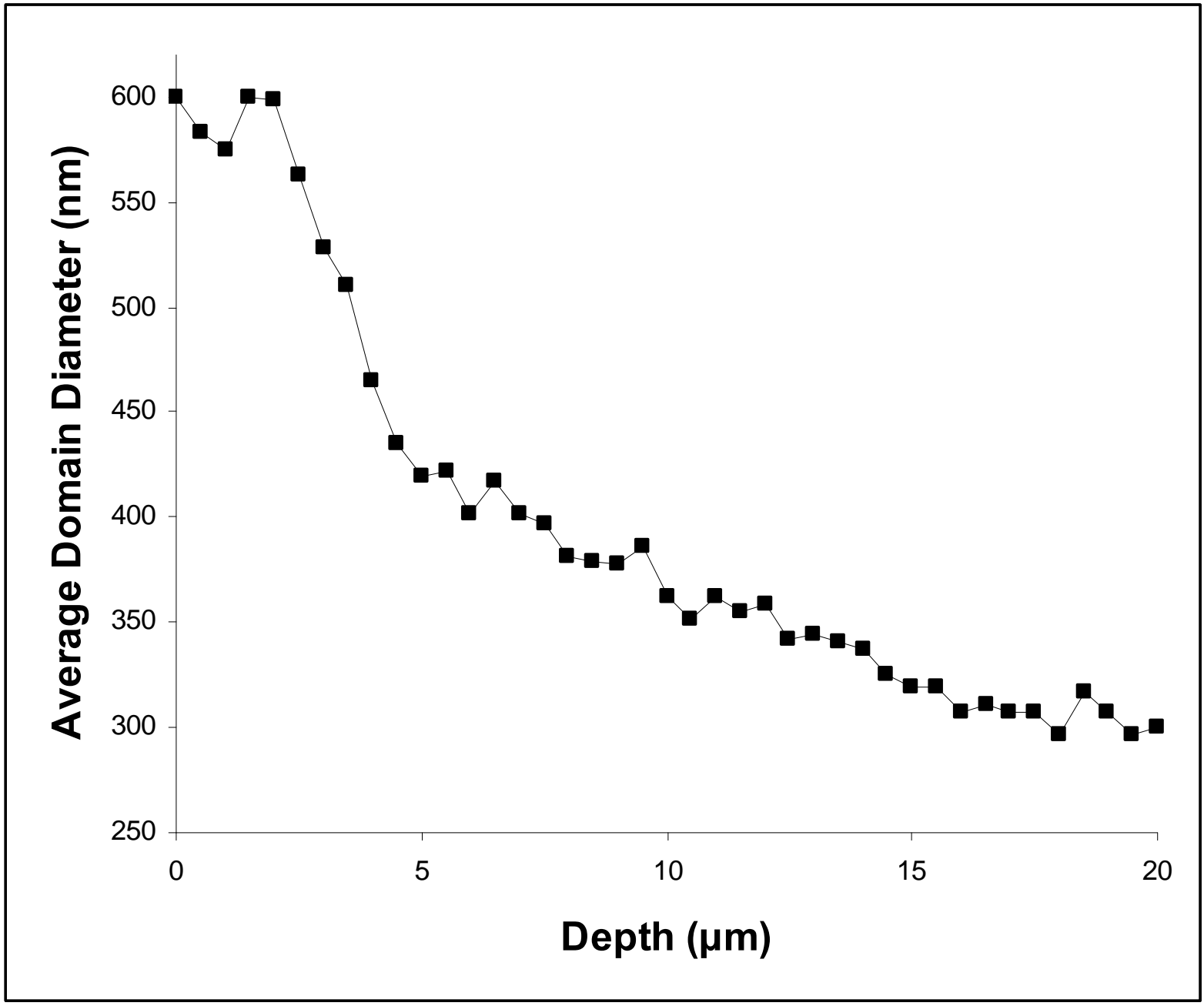

Figure 4-6: Average domain size depth profile for NR-IPN at equilibrium swelling, $5 \% \mathrm{v} / \mathrm{v}$ TEGDMA.

While the microemulsion provides a low viscosity bicontinuous template for the polymerization, the polymerization process itself is disruptive to this template. Changes in composition as monomer/prepolymer is consumed and changes in local temperature due to heats of reactions will affect the phase behavior, which in turn alters the structure. Regardless of how the polymerization is conducted, the microemulsion structure changes with time. Several approaches to counter this effect have been described above, such as partially quenching one 
phase so as to provide a higher viscosity template for the desired morphology, or introducing polymerizable surfactants. In simultaneous polymerization, the goal is to quench the desired morphology faster than the rate at which the microemulsion can equilibrate in response to changing conditions. The change in average NR-IPN domain size with respect to depth is expected as the impact of surface/interfacial effects is reduced ${ }^{11}$. However, LSCM imaging also shows that the domain size within each cross-section seen in Figure 4-5 is not uniform. This non-uniformity may be a result of changes in microemulsion phase behavior, and consequently the domain size of the template as the polymerization progresses. In effect, there is a gradient in the IPN domain size that depends on the remaining monomer/prepolymer at a given time - the reaction vessel and its contents are dynamic.

In contrast, the bulk microstucture seen in C-IPNs and D-IPNs at equilibrium swelling is both finer and more uniform than in NR-IPNs at 5\% v/v TEGDMA, as shown in Figure 4-7. Bicontinuous microemulsions are commonly associated with domain sizes of $<100 \mathrm{~nm}^{15-18,20}$. The persistence length for the microemulsion templates used in this work was estimated to be $\leq$ $36 \mathrm{~nm}$, corresponding to diameters of $\leq 72 \mathrm{~nm}$. As the image of IPNs presented here are at equilibrium swelling, we will need to evaluate the swelling behaviour in order to compare the persistence length with the expected IPN domain sizes. 
Figure 4-7: LSCM image of C-IPN (left) and D-IPN (right) at equilibrium swelling, 5\% v/v TEGDMA, $10 \mu \mathrm{m}$ depth.

\subsubsection{DSC and Texture Analysis}

A typical differential scanning calorimetry (DSC) curve comparing dried NR-IPNs at 5\% v/v TEGDMA to the PDMS and P(MAA-HEMA) controls used in formulation is presented in Figure 4-8. The DSC curve for the NR-IPN does not show the glass transition of P(MAAHEMA) at $285^{\circ} \mathrm{C}$, indicating that the hydrogel and PDMS components have not phase separated in the NR-IPN. The lack of a P(MAA-HEMA) glass transition temperature may be attributed to the restricted mobility of the hydrogel chains in the IPN structure. Li et al. ${ }^{48}$ and 
Haraguchi et al. ${ }^{49}$ have previously reported on the effects of steric hindrance on IPN thermal behaviour. The slope for NR-IPN curve is partway between that of the control hydrogel and control PDMS, consistent with behaviour reported by Liu et al. ${ }^{10}$.

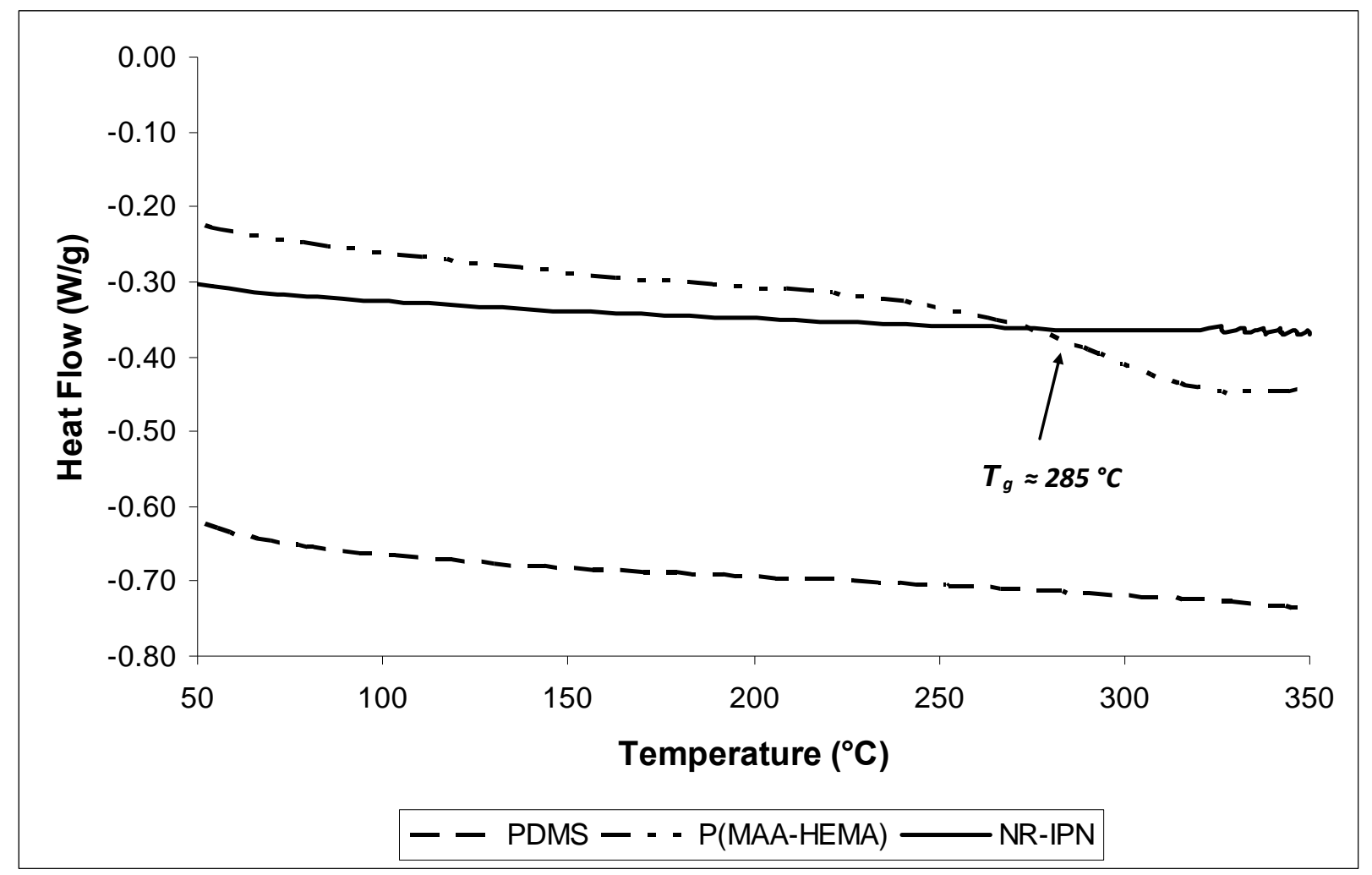

Figure 4-8: Sample DSC comparison of an NR-IPN (5\% v/v TEGDMA) with the PDMS and P(MAA-HEMA) (5\% v/v TEGDMA) components used in its formulation. DSC measurements are taken on dry samples.

Typical texture analysis measurements are presented in Figure 4-9a for sample IPNs (3\% v/v TEGDMA) at equilibrium hydration. A comparison of peak penetration forces between IPNs, control PDMS and control hydrogel (3\% v/v TEGDMA) at a depth of $5 \mathrm{~mm}$ is presented in Figure 4-9b. The relative forces, measured as a resistance to penetration by the cylindrical 
probe, are indicative of mechanical strength. The hydrated hydrogel, for example, offers little resistance. The hydrated NR-IPN, C-IPN and D-IPN reached normalized peak forces of 0.17 , 0.24 and 0.6 respectively when compared to the PDMS control. As described in the introduction, one of the goals in formulating silicone hydrogels is to impart the mechanical properties of PDMS to the final material, and this effect is evident in the texture analysis comparison. The normalized peak forces indicate that mechanical strengths of the C-IPNs and D-IPNs exceed that of the NR-IPN, which is consistent with the expected higher silicone content contributed by the reactive surfactants. However, as the silicone content in the C-IPN and D-IPN are expected to be equal, the difference in peak forces might suggest a higher degree of silicone crosslinking in the D-IPN. 

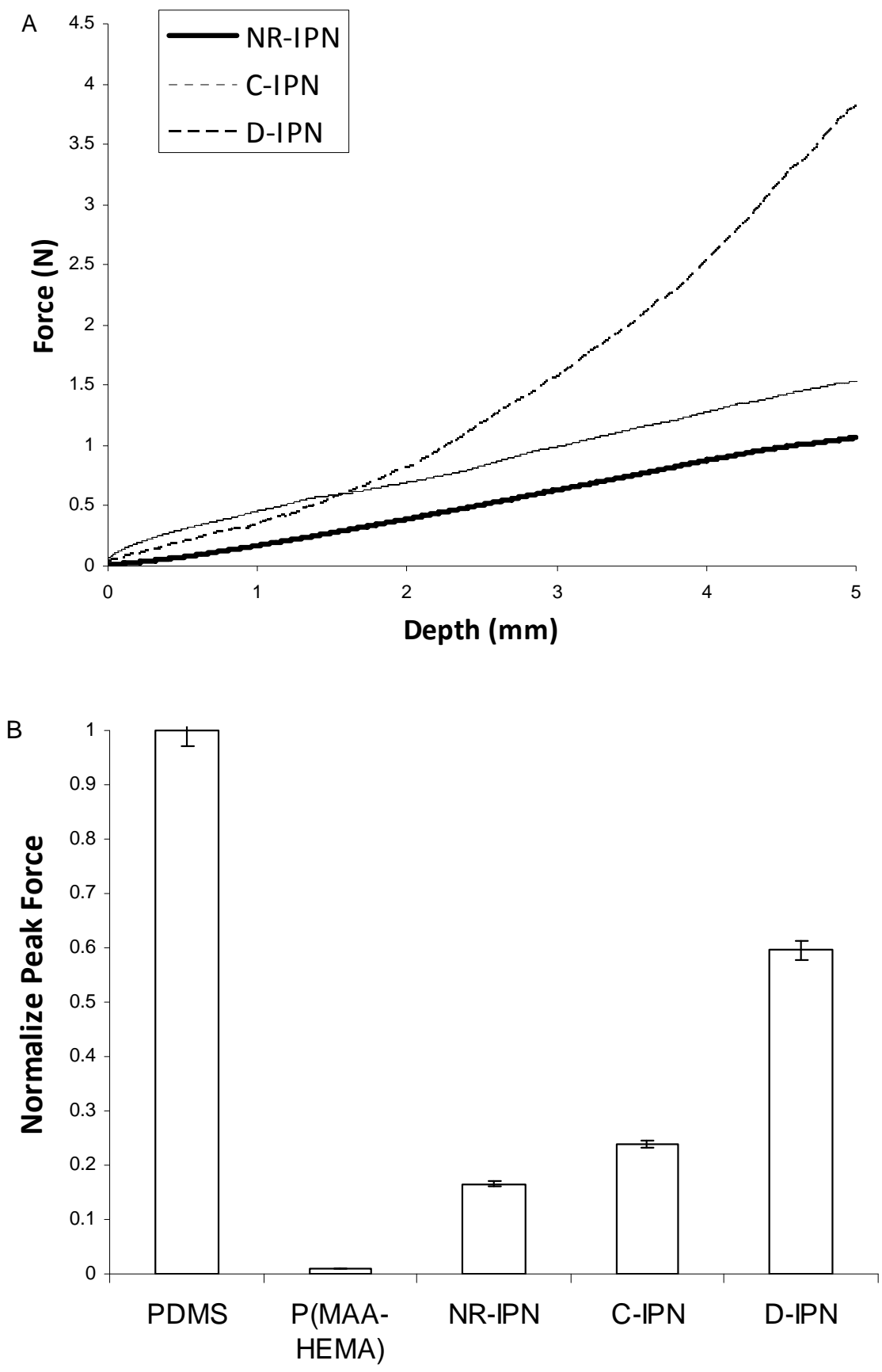

Figure 4-9: Texture analysis of microemulsion templated IPNs with $3 \%$ v/v TEGDMA. A) Force of compression/penetration through IPNs as a function of depth. B) Comparison of peak forces with control PDMS and P(MAA-HEMA), 3\% v/v TEGDMA. Peak forces are normalized to the peak force of compression/penetration for the PDMS control. 


\subsubsection{Permeability and Swelling Behavior}

A comparison of swelling behavior between NR-IPNs and the P(MAA-HEMA) control is presented in Figure 4-10 for varying amounts of the crosslinker, TEGDMA. A comparison of maximum hydration for non-reactive and reactive surfactant formulations is presented in Figure 4-11. Swelling kinetics and hydration in IPNs generated from each formulation are consistent with that of a hydrogel. From diffusion cell experiments, membrane permeability to vitamin $\mathrm{B}_{12}$ at $3 \% \mathrm{v} / \mathrm{v}$ TEGDMA was calculated to be $4.2 \pm 0.2 \times 10^{-6} \mathrm{~cm}^{2} / \mathrm{s}$ for NR-IPNs, $3.6 \pm 0.2 \times 10^{-6}$ $\mathrm{cm}^{2} / \mathrm{s}$ for C-IPNs and $9.8 \pm 0.6 \times 10^{-7} \mathrm{~cm}^{2} / \mathrm{s}$ for D-IPNs. To add context, Turner et al..$^{50}$ reported permeabilities of up to $6.7 \times 10^{-7} \mathrm{~cm}^{2} / \mathrm{s}$ for swollen PDMS-PMAA IPNs at $90 \%$ hydration $(30 \%$ PMAA on a dry mass basis). Estimates of the diffusion coefficient for vitamin $\mathrm{B}_{12}$ in water range from $3.79 \times 10^{-6} \mathrm{~cm}^{2} / \mathrm{s}$ to $4.4 \times 10^{-6} \mathrm{~cm}^{2} / \mathrm{s}$ in literature ${ }^{51-53}$. These results are summarized in Figure 4-12. The $\mathrm{B}_{12}$ permeabilities for the NR-IPN and C-IPN and the $\mathrm{B}_{12}$ diffusion coefficients in water obtained from literature are not significantly different $(\mathrm{p}<0.05)$, indicating partition coefficients of close to 1 . This result is not surprising, as swollen NR-IPNs at $3 \% \mathrm{v} / \mathrm{v}$ TEGDMA increased in volume by a factor of 14.3 at equilibrium swelling, indicating that approximately $93 \%$ of the swollen volume is water. By contrast, $87 \%$ of the swollen volume in D-IPNs is water, but the partition coefficient is approximately 0.24 . This may indicate that aqueous channel continuity in NR- and C-IPNs exceeds that of D-IPNs. 


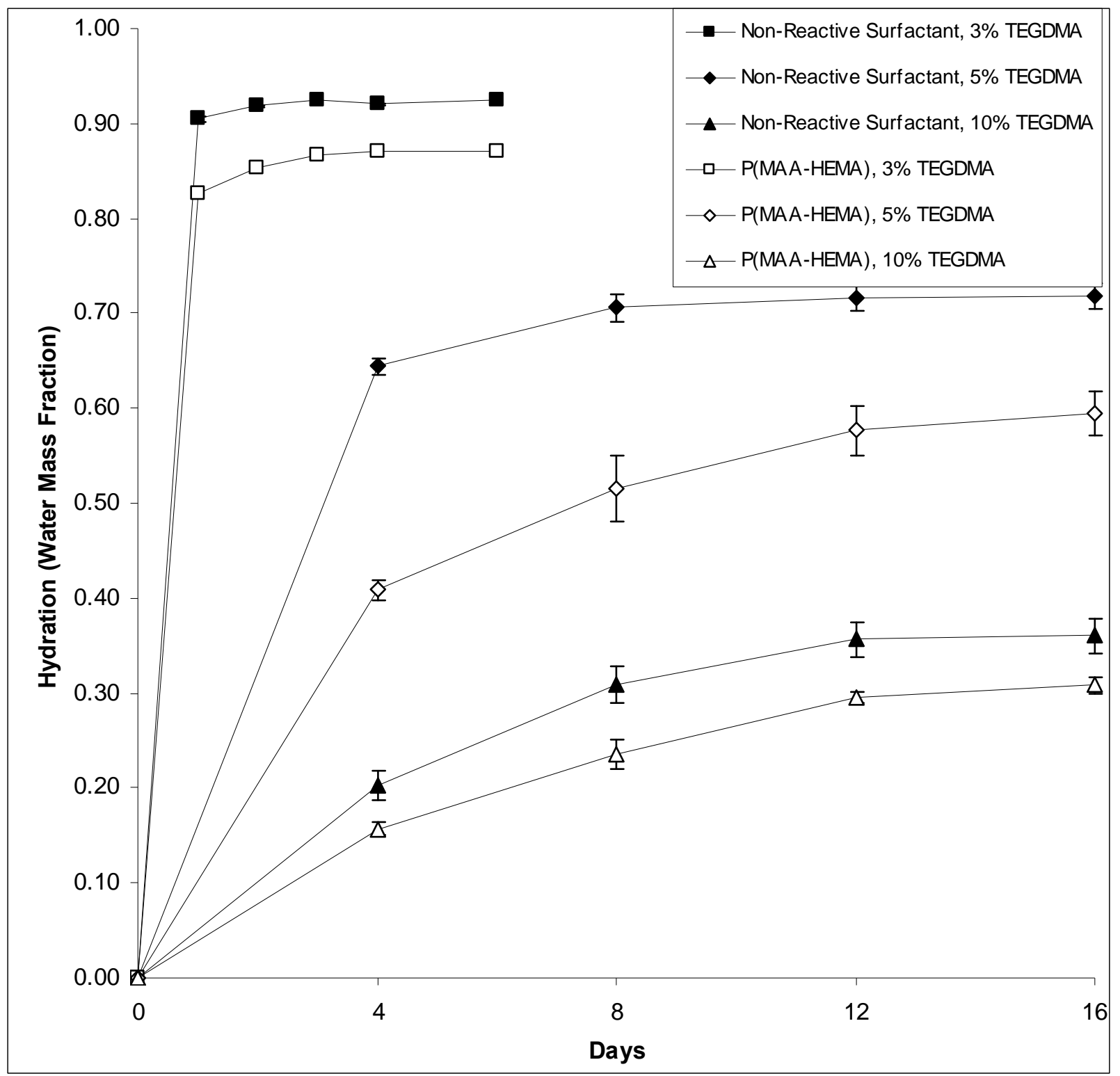

Figure 4-10: Swelling behavior for polymers formed from silicone microemulsions with non-reactive surfactants at $3 \%, 5 \%$ and $10 \% \mathrm{v} / \mathrm{v}$ (HEMA/MAA) TEDGMA. $N=4$. Hydration is calculated as water mass/total swollen mass. Control hydrogel is polymerized from the aqueous mixtures used in the microemulsion-templated IPN. 


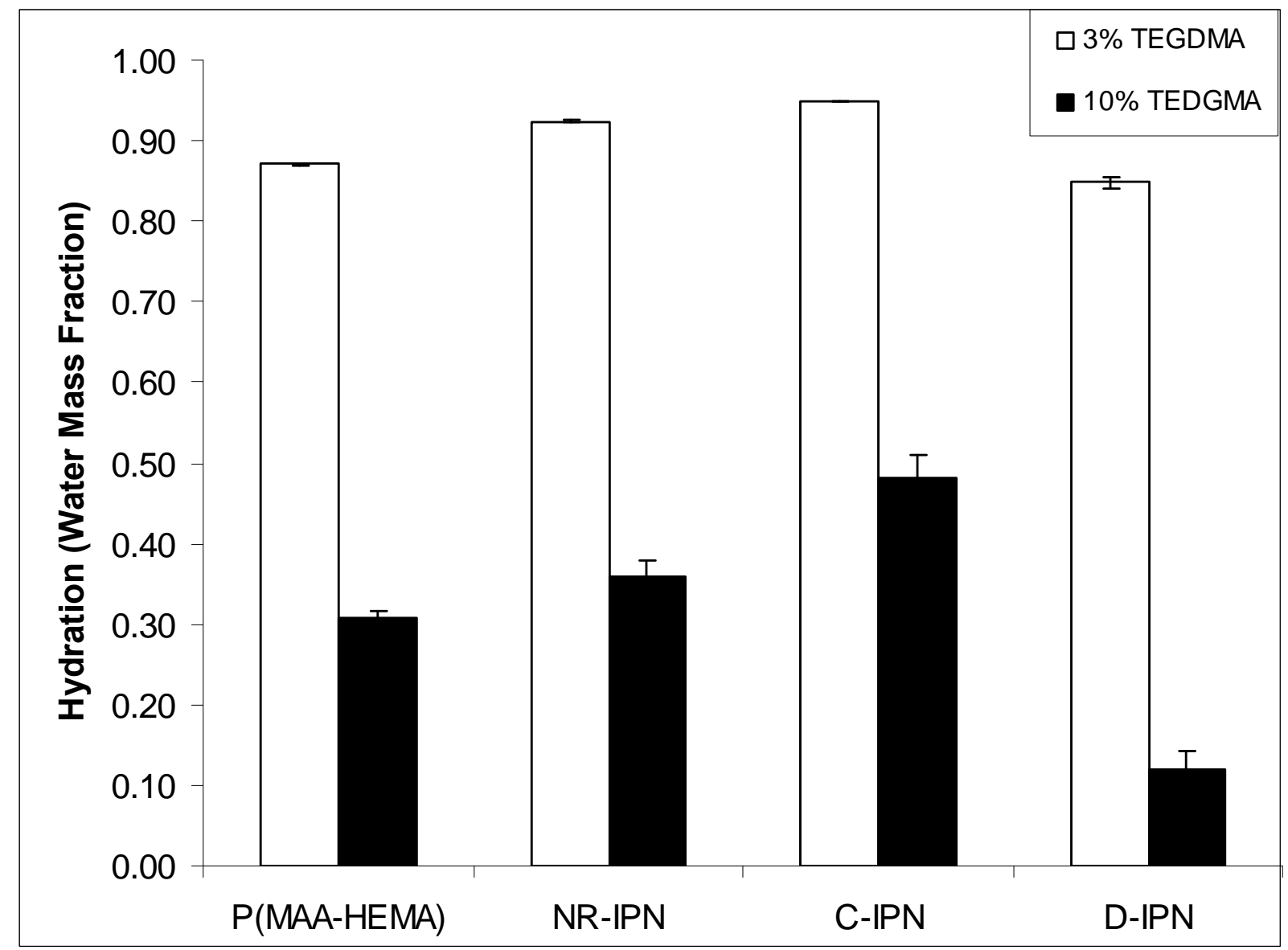

Figure 4-11: Comparison of maximum hydration for IPNs formed from formulations with and without polymerizable surfactant at $3 \%$ and $10 \%$ v/v (HEMA, MAA) TEGMA. N=4. Control hydrogel is polymerized from the aqueous mixtures used in the microemulsiontemplated IPN. 


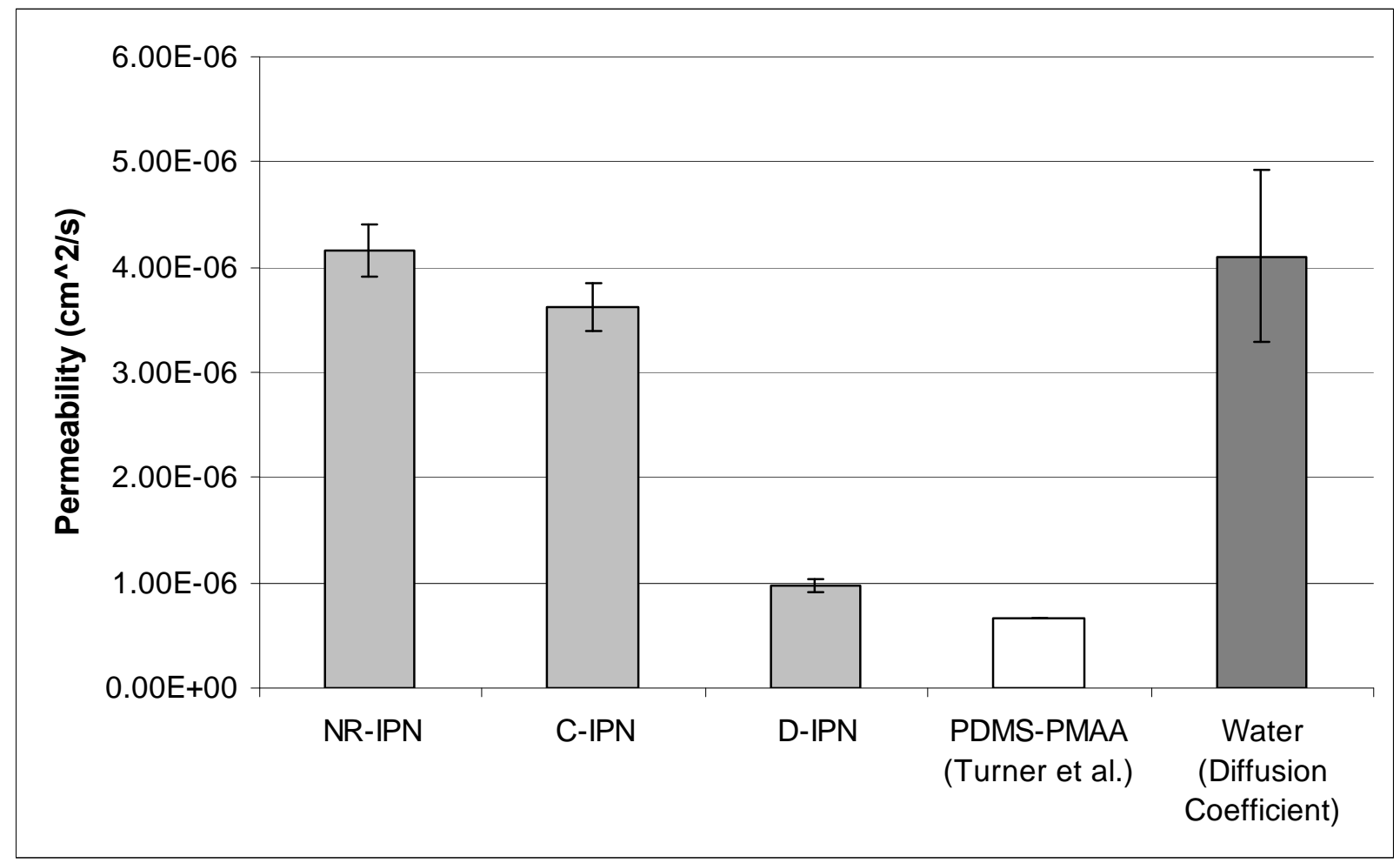

Figure 4-12: Comparison of vitamin $B_{12}$ permeabilities $(n=4)$ for IPNs at $3 \% \mathrm{v} / \mathrm{v}$ TEGDMA with PDMS-PMAA (Turner et al. ${ }^{50}, 30 \%$ PMAA) and estimates for the diffusion coefficient of $B_{12}$ in water ${ }^{51-53}$.

It is interesting to note that although MAA and HEMA make up $\sim 64 \%$ of polymerizable mass in the non-reactive surfactant formulations, and $\sim 40 \%$ of polymerizable mass in the reactive surfactant formulations, the NR-IPNs and C-IPNs consistently showed higher levels of hydration based on total mass than the control P(MAA-HEMA) hydrogels at the same theoretical crosslinking density. A higher degree of hydration is normally associated with lower effective crosslinking density. The lower hydration and permeability reported for D-IPNs can be attributed to surfactant $\mathrm{D}$ being multifunctional. Molecules of surfactant $\mathrm{C}$ (linear difunctional, acrylate end groups) can have 0,1 or 2 polymerizable acrylate groups, with an average of 1.5. On the other hand, molecules of surfactant D (multifunctional, acrylate pendant 
groups) nominally contain an average of 2 acrylate groups, but manufacturing limitations suggest that a range can be expected ${ }^{34}$. The swelling behavior suggests that surfactant $\mathrm{D}$ acts as an additional crosslinker (increasing the effective crosslinking density), while surfactant $\mathrm{C}$ may behave as a chain extender and terminator (decreasing the effective crosslinking density). Furthermore, permeability data indicates that surfactant $\mathrm{D}$ may play a role in reduced aqueous continuity. In either case, the swelling of IPNs formed from microemulsion templates was not expected to exceed that of the control P(MAA-HEMA) hydrogels on a P(MAA-HEMA) mass basis, and indicates a much lower effective crosslinking density than in the controls. A plausible explanation might be that the crosslinking agent, TEDGMA, partitions into either the oil or surfactant phase during equilibration. However, the crosslinker was found to be insoluble in both the oil and the surfactants.

While P(MAA-HEMA) is hydrophilic, the monomers MAA and TEGDMA are known to readily swell crosslinked PDMS ${ }^{8,11,12}$, providing a potential mechanism for loss of monomer and crosslinker from the aqueous phase. In order to test this hypothesis, $100 \mathrm{mg}$ samples of crosslinked PDMS were swollen in TEGDMA, yielding an equilibrated swollen mass of $128 \pm 4$ $\mathrm{mg}(\mathrm{n}=10)$. This effect is compounded by the relatively low solubility of TEGDMA in water compared to MAA and HEMA. Thus monomer consumption and MAA migration may lead to a reduction in the solubility of TEGDMA in the aqueous phase, and may promote partitioning of TEGDMA into the silicone rubber. This segregation of reactive components as polymerization proceeds may provide an explanation for both the lower effective crosslinking and the variation in NR-IPN domain size observed. Also, we speculate that as the mediating connections between the aqueous phase and surfactant formed through MAA and HEMA are eliminated, there is a 
corresponding shift in the phase behavior of the remaining liquid phases away from the initial bicontinuous morphology. The polymerizable surfactants may aid in stabilizing the initial structure by increasing the viscosity of the formulation during the polymerization process, as demonstrated by the more uniform swollen IPNs. However, this does not prevent the partitioning of MAA, HEMA and TEGMA out of aqueous solution.

Finally, by evaluating the swelling data, domains sizes obtained through LSCM and the calculated persistence length, we can compare the IPN morphology to that of the microemulsion template. Swollen NR-IPNs at $5 \% \mathrm{v} / \mathrm{v}$ TEGDMA increased in volume by a factor of $\sim 3.5$, while C-IPNs increased in volume by a factor of $\sim 5$. With the simplifying assumption that the IPNs swell evenly, we would expect swollen characteristic lengths (radius) of $<55 \mathrm{~nm}$ in NR-IPNs and $<62 \mathrm{~nm}$ in C-IPNs. These estimates are consistent with the domains seen for C-IPNs in figure 6, though the NR-IPNs in figure 4 had characteristic lengths (radius) of $\geq 150 \mathrm{~nm}$. While polymerizable surfactants have previously been reported as helping to preserve microemulsion structure and domain size in polymerized micellar w/o systems ${ }^{28}$, this result supports the hypothesis that polymerizable surfactants can also be used to limit changes in the domain sizes expected in a bicontinuous microemulsion.

\subsection{CONCLUDING REMARKS}

This chapter presents a simple process for simultaneous polymerization of PDMS-P(MAAHEMA) IPNs templated on bicontinuous microemulsions and stabilized by silicone alkyl 
polyether and silicone acrylate surfactants. Previously, combining hydrophilic components with PDMS has required sequential polymerization with multiple curing and equilibration steps ${ }^{8-12}$. Successful polymerization of bicontinuous formulations has required using one phase as a high viscosity scaffold for polymerization of a second phase ${ }^{29-32}$. We have shown that with appropriate characterization and selection of components ${ }^{33}$, we can formulate a low viscosity, reactive bicontinuous microemulsion that yields a hydrogel-PDMS IPN in one polymerization step. The swelling behavior and permeability of the resulting polymer materials indicate the formation of a hydrogel phase. LSCM on IPNs swollen in sodium fluorescein solution confirms connectivity in the aqueous phase, and also confirms that both phases are well distributed with swollen domain sizes of $<1 \mu \mathrm{m}$. Furthermore, we have shown that the introduction of polymerizable surfactants can aid in stabilizing and preserving the structure of the bicontinuous microemulsion template, reducing swollen domain size to $<200 \mathrm{~nm}$. Previous reports in the literature had focused on micellar w/o systems ${ }^{28}$. 


\subsection{REFERENCES}

1. Sperling, L. H.; Mishra, V. Polym. Adv. Technol. 1996, 4, 197-208.

2. Lucas, P.; Robin, J. -J. Advances in Polymer Science 2007, 1, 111-147.

3. Kim, S. C.; Klempner, D.; Frisch, K. S.; Radigan, W.; Frisch, H. L. Macromolecules 1976, 2, 258-263.

4. Klempner, D.; Frisch, K. C. In Advances in Interpenetrating Polymer Networks. Technomic: Lancaster, PA, 1994.

5. Lee, J. H.; Kim, S. C. Macromolecules 1986, 3, 644-648.

6. Sperling, L. H. Interpenetrating Polymer Networks and Related Materials; Plenum: New York, 1981.

7. Zhang, X.; Wu, D.; Chu, C. Biomaterials 2004, 17, 3793-3805.

8. Abbasi, F.; Mirzadeh, H.; Katbab, A. A. J Appl Polym Sci 2002, 9, 1825-1831.

9. Pavlyuchenko, V. N.; Sorochinskaya, O. V.; Ivanchev, S. S.; Khaikin, S. Y.; Trounov, V. A.; Lebedev, V. T.; Sosnov, E. A.; Gofman, I. V. Polym. Adv. Technol. 2009, 4, 367-377.

10. Liu, L.; Sheardown, H. Biomaterials 2005, 3, 233-244.

11. Turner, J. S.; Cheng, Y. -L. Macromolecules 2000, 10, 3714-3718.

12. Turner, J. S.; Cheng, Y. -L. Macromolecules 2003, 6, 1962-1966.

13. Hoar, T. P.; Schulman, J. H. Nature 1943, 3847, 102-103.

14. Danielsson, I.; Lindman, B. Colloids and Surfaces 1981, 4, 391-392.

15. Winsor, P. A. Solvent Properties of Amphiphilic Compounds; Butterworths: London, 1954; .

16. Winsor, P. A. Transactions of the Faraday Society 1948, 376-398.

17. Salager, J.; Antón, R. E.; Sabatini, D. A.; Harwell, J. H.; Acosta, E. J.; Tolosa, L. I. J Surfact Deterg 2005, 1, 3-21.

18. Holmberg, K. Microemulsions in Biotechnology. In Industrial Application of Microemulsions; Solans, C., Kunieda, H., Eds.; Marcel Dekker: New York, 1997; pp 69.

19. Lawrence, M. J.; Rees, G. D. Advanced Drug Delivery Reviews 2000, 1, 89-121.

20. Chow, P. Y.; Gan, L. M. Microemulsion polymerizations and reactions. In Polymer Particles; Okubo, M., Ed.; Springer-Verlag: Berlin, 2005; Vol. 175, pp 257.

21. Chew, C. H.; Li, T. D.; Gan, L. H.; Quek, C. H.; Gan, L. M. Langmuir 1998, 21, 6068-6076.

22. Guerrero-Ramírez, L. G.; Nuño-Donlucas, S. M.; Cesteros, L. C.; Katime, I. Materials Chemistry and Physics 2008, 3, 1088-1092. 
23. O’Donnell, J.; Kaler, E. W. Macromolecules 2008, 16, 6094-6099.

24. Jones, B. H.; Lodge, T. P. Chemistry of Materials 2010, 4, 1279-1281.

25. Yang, J.; Shu, W. -B.; Qin, W. Gongneng Cailiao/Journal of Functional Materials 2010, 1, 148-152.

26. Peinado, C.; Bosch, P.; Martin, V.; Corrales, T. J. Polym. Sci. Part A 2006, 18, 5291-5303.

27. Wang, H. -J.; Li, D.-S.; Peng, M. Gaofenzi Cailiao Kexue Yu Gongcheng/Polymeric Materials Science and Engineering 2008, 12, 70-73.

28. Summers, M.; Eastoe, J.; Davis, S.; Du, Z.; Richardson, R. M.; Heenan, R. K.; Steytler, D.; Grillo, I. Langmuir 2001, 17, 5388-5397.

29. Gao, F.; Ho, C.; Co, C. C. Macromolecules 2006, 26, 9467-9472.

30. Stubenrauch, C.; Tessendorf, R.; Strey, R.; Lynch, I.; Dawson, K. A. Langmuir 2007, 14, $7730-7737$.

31. Stubenrauch, C.; Tessendorf, R.; Salvati, A.; Topgaard, D.; Sottmann, T.; Strey, R.; Lynch, I. Langmuir 2008, 16, 8473-8482.

32. Magno, M.; Tessendorf, R.; Medronho, B.; Miguel, M. G.; Stubenrauch, C. Soft Matter 2009, 23, 4763-4772.

33. Castellino, V.; Cheng, Y.; Acosta, E. J. Colloid Interface Sci. 2010, 353, 196-205.

34. Siltech Corporation http://www.siltechcorp.com.

35. Acosta, E.; Uchiyama, H.; Sabatini, D. A.; Harwell, J. H. Journal of Surfactants and Detergents 2002, 2, 151-157.

36. Acosta, E. J.; Harwell, J. H.; Sabatini, D. A. J. Colloid Interface Sci. 2004, 2, 652-664.

37. Acosta, E. J.; Nguyen, T.; Witthayapanyanon, A.; Harwell, J. H.; Sabatini, D. A. Environmental Science and Technology 2005, 5, 1275-1282.

38. Lim, W. H. J Surfact Deterg 2006, 4, 349-355.

39. Meier, W. Colloid Polym. Sci. 1997, 6, 530-536.

40. Laguës, M.; Sauterey, C. J. Phys. Chem. 1980, 26, 3503-3508.

41. Clausse, M.; Peyrelasse, J.; Heil, J.; Boned, C.; Lagourette, B. Nature 1981, 5834, 636-638.

42. De Gennes, P. G.; Taupin, C. J. Phys. Chem. 1982, 13, 2294-2304.

43. Sottmann, T.; Strey, R.; Chen, S. -H. J. Chem. Phys. 1996, 15, 6483-6491.

44. Zemb, T. Comptes Rendus Chimie 2009, 1-2, 218-224.

45. Guest, D.; Auvray, L.; Langevin, D. Journal de physique.Lettres 1985, 22, 1055-1063.

46. Gentle, T. E.; Snow, S. A. Langmuir 1995, 8, 2905-2910.

47. Rosen, M. J. Surfactants and Interfacial Phenomena, 3rd edition; John Wiley and Sons: New York, 2004

48. Li, B.; Jiang, Y.; Liu, Y.; Wu, Y.; Yu, H.; Zhu, M. J. Polym. Sci. Part B 2009, 1, 96-106.

49. Haraguchi, K.; Li, H. -J.; Song, L.; Murata, K. Macromolecules 2007, 19, 6973-6980. 
50. Turner, J. S.; Cheng, Y. -L. J.Membr.Sci. 2004, 2406, 19-24.

51. Gehrke, S. H.; Cussler, E. L. Chemical Engineering Science 1989, 3, 559-566.

52. Dembczynski, R.; Jankowski, T. Biochem. Eng. J. 2000, 1, 41-44.

53. Colton, C. K.; Smith, K. A.; Merrill, E. W.; Farrell, P. C. J. Biomed. Mater. Res. 1971, 5 , 459-488. 


\section{MICROEMULSION PHASE BEHAVIOUR AND POLYMER STRUCTURE}

While the primary focus of this work is the polymerization of a silicone-hydrogel IPN, it is also interesting to investigate the materials generated from other regions in the microemulsion ternary phase diagram. In this chapter, we will explore the hypothesis that:

\section{If the composition of the microemulsion template is changed, then the nanostructure of the corresponding polymer will change to reflect the template.}

This hypothesis will be discussed in light of changes in the oil/aqueous ratio, microemulsion and polymer morphology, and most importantly, changes in the surfactant content. Materials were formulated and polymerized as described in chapter 4.2.4, and were imaged at equilibrium swelling in sodium fluorescein solution to highlight aqueous pathways. Imaging was conducted using an inverted Olympus Fluoview 300 with $488 \mathrm{~nm}$ excitation, 25\% laser power, FITC filter cube and a 1.4 NA oil immersion objective. Images were captured at 512 x 512 resolution in 8bit gray scale. Materials are defined and labeled according to their surfactant: oil: aqueous ratio (x: y: z) and surfactant type: NR- (non-reactive), C- (surfactant C) and D- (surfactant D). Samples contained 10\% v/v (7\% mol/mol) TEGDMA. Images are taken at a depth of $15 \mu \mathrm{m}$.

Reports on the polymerization of bicontinuous systems in literature can provide some context for the link between microemulsion and polymer morphology. Attempts to polymerize bicontinuous microemulsions by Peinado et al. ${ }^{1}$ led to phase separation and a breakdown of 
microemulsion structure as monomer was consumed. However, they observed the formation of a beaded-wall structure, where spherical domains were found to remain connected by thin channels. They hypothesized that the bicontinuous structure was partially lost as crosslinking and gelation occurred. The inability to preserve the interface or maintain a bicontinuous structure led to domain sizes of 2.3-2.8 $\mu \mathrm{m}$. By contrast, approaches that take advantage of high viscosity templates have had more success. Stubenrauch et al. ${ }^{2,3}$ and Magno et al. ${ }^{4}$ successfully constructed bicontinuous microemulsions with one polymerizable phase containing NIPAAM by introducing a gelling agent to the oil phase, obtaining a persistence length of $\sim 22 \mathrm{~nm}$ with a semi-rigid oil interface. Gao et al. ${ }^{5}$ used a glassy sugar template to obtain domain sizes on the order of $\sim 25 \mathrm{~nm}$. Summers et al. ${ }^{6}$ reported on the use of polymerizable surfactants to stabilize water-in-oil microemulsions, finding that these aided greatly in preserving micellar domain sizes on the order of $<10 \mathrm{~nm}$. However, there are yet to be reports of microemulsion polymerization where both phases in a bicontinuous formulation have had their structures preserved.

\subsection{THE INFLUENCE OF MICROEMULSION MORPHOLOGY AND PHASE BEHAVIOUR ON POLYMER MORPHOLOGY}

Figure 5-1 is an LSCM image of the hydrogel-silicone boundary in a formulation with 50\% aqueous solution and $50 \%$ oil (0:5:5), providing a point of reference. In an ideal polymerization setting, the high interfacial tension between the oil and aqueous phase would lead to a distinct, flat boundary. However, the boundary appears rough and uneven at a depth of $15 \mu \mathrm{m}$, with penetration of the sodium fluorescein into the PDMS phase. The disruptive polymerization process and monomer migration are both potential mechanisms that could contribute to this 
effect. The image provides some context for these assertions, made in chapter 4.3.3, offering a larger scale view of the effects at the oil-aqueous interface.

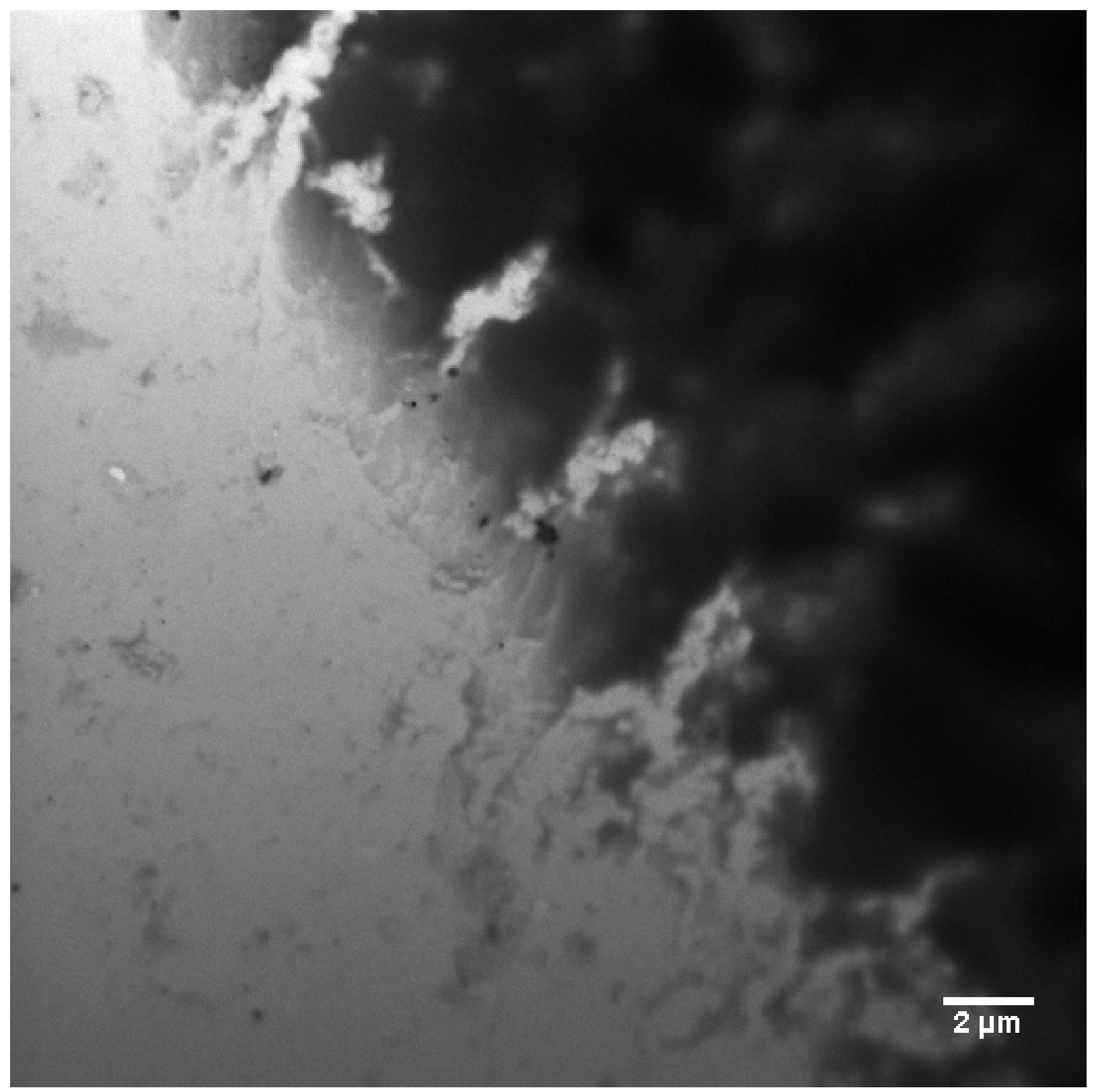

Figure 5-1 LSCM image of hydrogel-PDMS boundary in 0:5:5 formulation. The highlighted regions indicate the presence of sodium fluorescein, $15 \mu \mathrm{m}$ depth. 


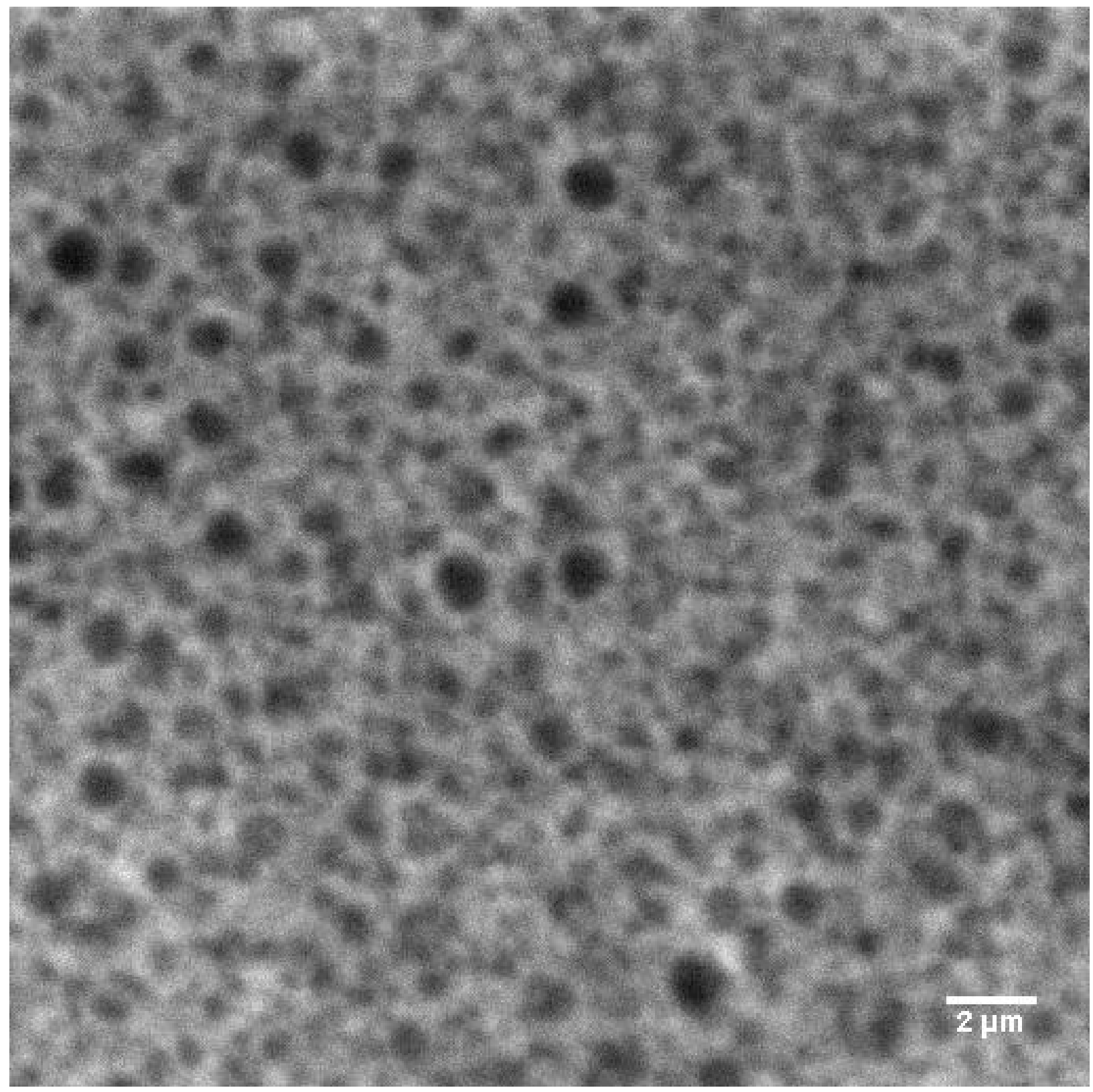

Figure 5-2: LSCM image of 3:2:5 NR-IPN swollen in sodium fluorescein solution, $15 \mu \mathrm{m}$ depth.

Figure 5-2 is an LSCM image of the 3:2:5 NR-IPN formulation evaluated in chapter 4 at 10\% v/v TEGDMA. The ratio of hydrogel to PDMS is approximately 5:4 by volume and 11:8 by mass based on initial monomer content. It is important to note that both the silicone and 
hydrogel domains are likely distorted by swelling of the hydrogel component, so that the swollen morphology may not reflect the dry morphology. The result is that the image appears to be saturated by a swollen hydrogel containing smaller PDMS domains.

While the 3:5:2 NR formulation exists as a lower phase microemulsion with excess oil at $25^{\circ} \mathrm{C}$, it exists as a homogeneous microemulsion at $55^{\circ} \mathrm{C}$, as shown in Figure 4-2. Interestingly, polymerization yields two materials corresponding to the microemulsion and excess oil phases in the initial formulation, as seen in Figure 5-3. Figure 5-4 is an example of the silicone-PDMS blend microstructure extracted from the lower phase. In contrast to the NR-IPN in Figure 5-2, this polymer blend contains larger, discrete PDMS domains (or PDMS-encapsulated hydrogel domains), as would be expected in a lower phase microemulsion with high silicone content. The polymer blend is opaque and white even when hydrated, which can be attributed to the large PDMS domains. The microstructure provides additional supporting evidence for the hypothesis that a polymerization-induced increase in viscosity arrests changes in phase behaviour, as the final material reflects the expected $25^{\circ} \mathrm{C}$ microemulsion morphology rather than the $55^{\circ} \mathrm{C}$ morphology. 


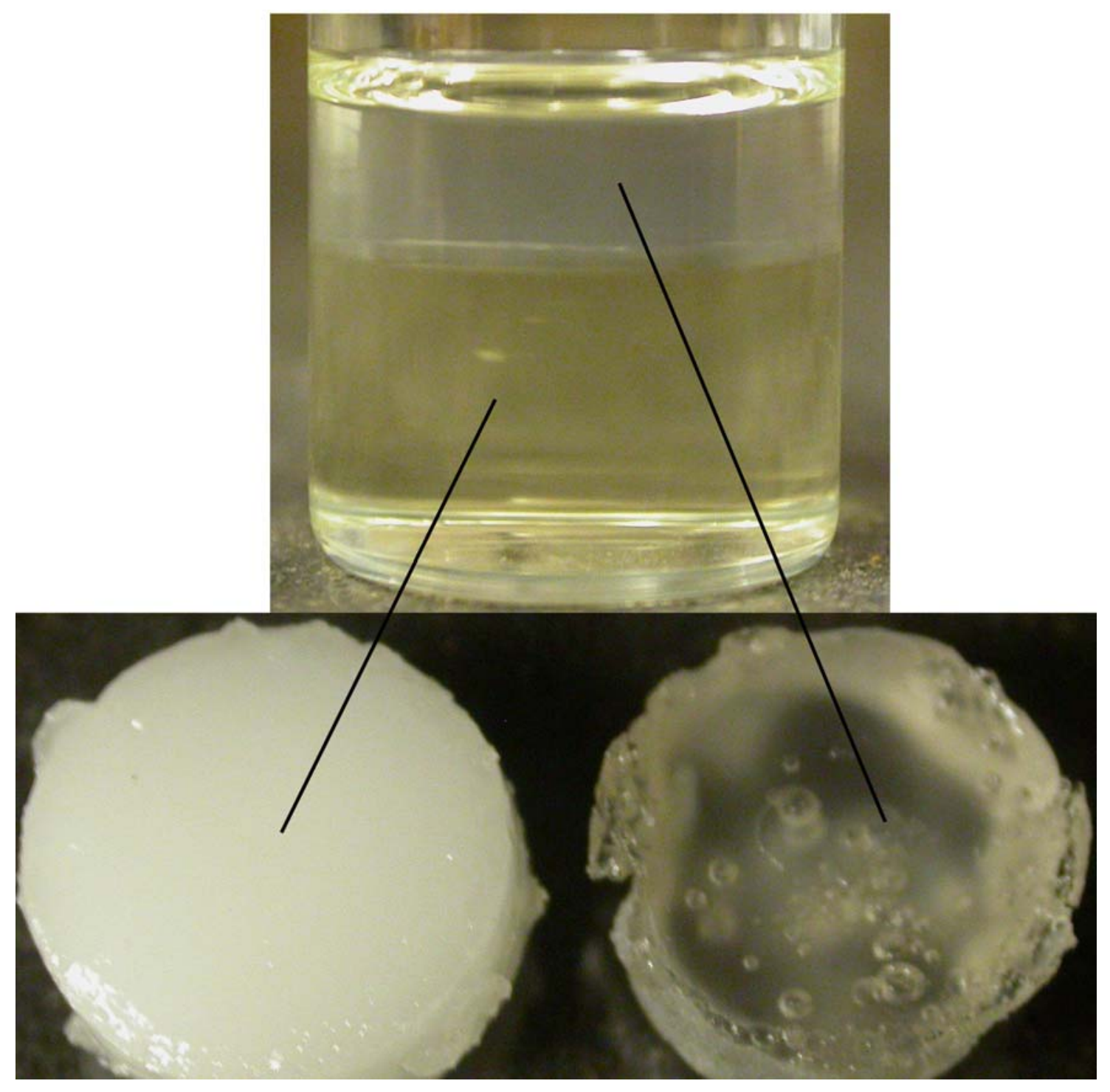

Figure 5-3: Comparison of materials polymerized from microemulsion (lower phase) and excess oil (upper phase) in 3:5:2 NR formulation. 


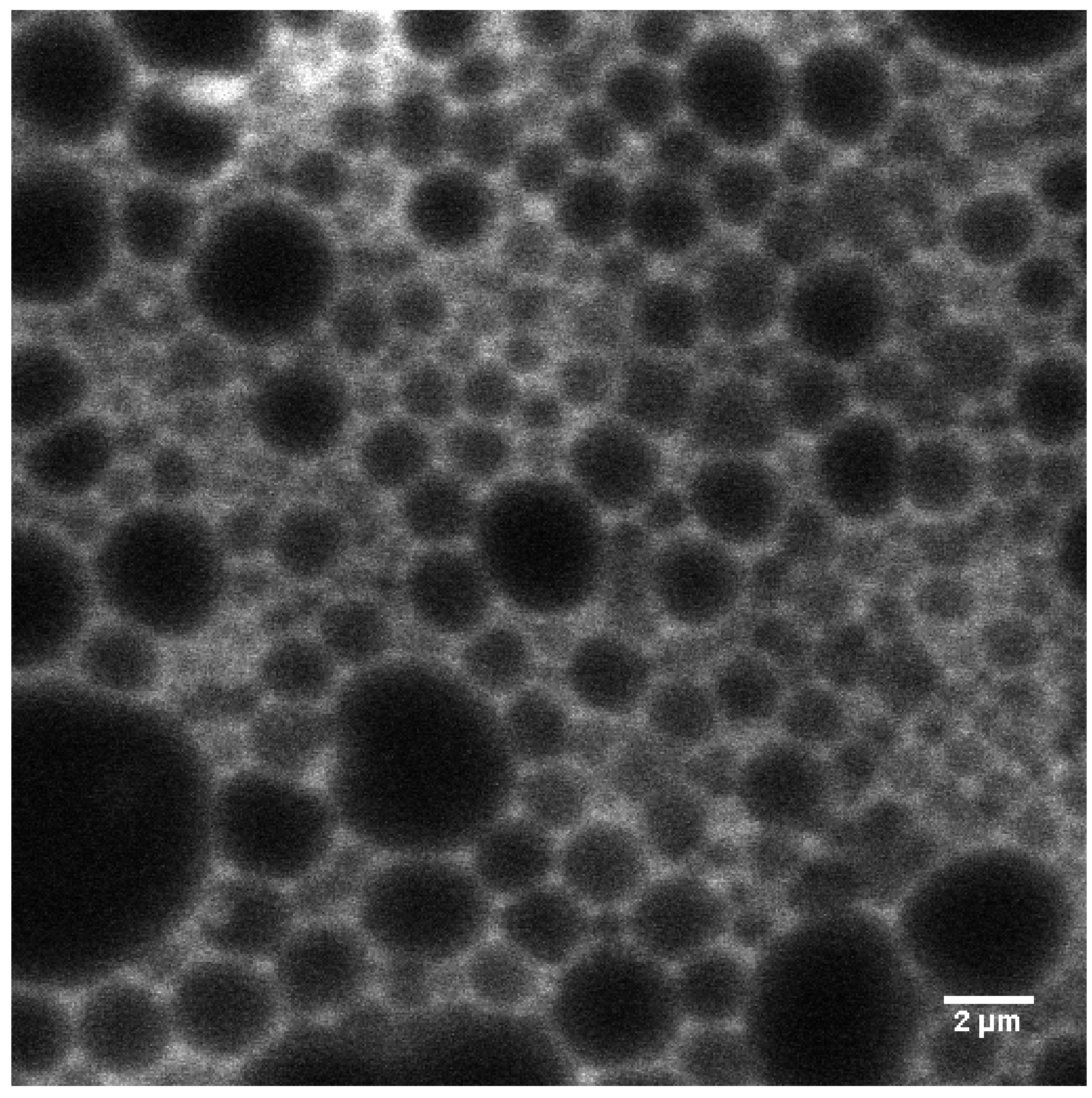

Figure 5-4: LSCM image of 3:5:2 NR- Polymer blend swollen in sodium fluorescein solution, $15 \mu \mathrm{m}$ depth. This formulation begins as a lower phase microemulsion at $25{ }^{\circ} \mathrm{C}$, and the material shown here is extracted from that region. 


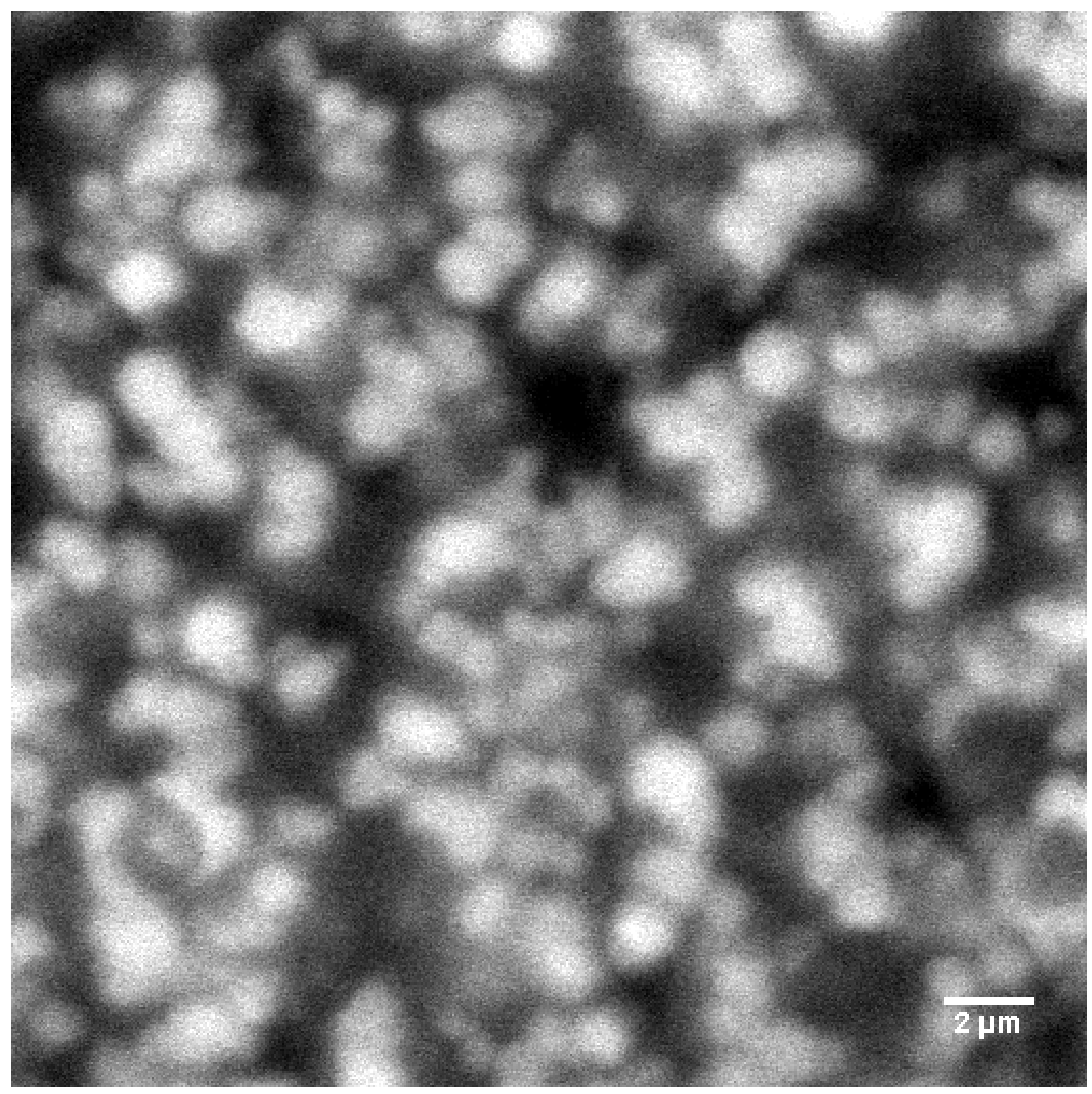

Figure 5-5: LSCM image of 2:1:1 NR-IPN swollen in sodium fluorescein solution, $15 \mu \mathrm{m}$ depth.

Figure 5-5 is an LSCM image of a swollen 2:1:1 NR-IPN. The initial formulation was homogeneous, and conductance measurement indicated a bicontinuous structure. The resulting swollen material consists of irregular silicone and hydrogel domains. The large highlighted regions indicate sodium fluorescein accessibility, in turn suggesting that hydrogel domains are 
connected. The irregular morphology seen in Figure 5-5 contrasts sharply with morphologies seen in materials generated from other regions in the ternary phase diagram. One would expect the silicone and aqueous domain sizes to decrease with higher surfactant content, yielding an estimated persistence length of $22 \mathrm{~nm}$. However, the swollen hydrogel and the PDMS domains appear significantly larger than in both the 3:2:5 NR-IPN (Figure 5-2) and the 3:5:2 polymer blend (Figure 5-4).

\subsubsection{Water Contact Angle Measurement}

Water contact angle measurements were taken by depositing a $50 \mu \mathrm{L}$ droplet of water onto 28.3 $\mathrm{cm}^{2}$ sample membranes. As expected from swelling studies and LSCM imaging, the contact angles were transient, indicating that the materials are hydrophilic with a contact angle of $0^{\circ}$. The range of materials tested included formulations with up to $70 \%$ surfactant, which suggests that even if the surfactant is the continuous phase in the microemulsion template, the resulting material remains sufficiently hydrophilic to maintain a $0^{\circ}$ water contact angle. Materials with greater than $80 \%$ silicone oil content in the microemulsion were not formulated or tested, though it could be expected that these materials would be significantly more hydrophobic. 


\subsection{ON POTENTIAL LINKS BETWEEN PHASE BEHAVIOUR, POLYMER STRUCTURE, AND POLYMER PROPERTIES}

In interpreting the swelling behaviour and changes in morphology, it is important to consider how the theoretical nanostructure of the microemulsion templated IPN differs from conventional silicone hydrogels formed by swelling silicone rubber in a guest monomer solution. Through the conventional route, the host network and guest monomer solution begin intimately mixed. Guest domains are formed as the guest monomer polymerizes and aggregates, known as polymerization-induced phase separation (PIPS). However, phase separation and the resulting increase in domain size are arrested by a corresponding increase in viscosity ${ }^{7}$. In the microemulsion-templated network, the initial template consists of distinct aqueous and oil domains separated by a surfactant interfacial layer, as opposed to a solvent. Similar to conventional synthesis, an increase in viscosity as polymerization proceeds was shown to arrest phase separation and increases in domain size. As previously described, the microemulsion templated materials generated here require an extension to the classical definition of an IPN. While conventional IPNs progress from a state of intimate mixing to a state with defined domains, microemulsion templated IPNs progress from defined domains in a liquid state to defined domains in a solid state. As a result, the scale of entanglement for microemulsion templated IPNs is expected to be on the order of clusters of polymer chains. By analogy, conventional IPNs could be compared to an entanglement of individual filaments, while microemulsion templated IPNs could be compared to an entanglement of ropes. In addition, the nature of the interfacial layer and contribution of the surfactant to the final structure remain critical differences between conventional and microemulsion-templated synthesis. 
In polymers generated from microemulsions containing the non-reactive surfactant blend, we attempt to remove the surfactant through a series of washing steps. While pockets of encapsulated surfactant may remain, the washing removes a significant volume of material. In the 3:2:5 NR formulation described in chapter 3, for example, the surfactant $(30 \%)$ and water $(22 \%)$ make up $52 \%$ of the initial volume. In the $2: 1: 1 \mathrm{NR}$ formulation described above, the surfactant (50\%) and water (11\%) account for $\sim 61 \%$ of the initial volume. In NR-IPNs, one could consider the surfactant a spacer between the aqueous and PDMS polymer channels, similar to the technique of dissolving one component in a solvent to promote a more open network as described by Liu et al. ${ }^{8}$. This effect is presented schematically in Figure 5-6.

Surfactant removal and the resulting loss of volume could be considered as essentially the opposite of swelling the IPN, leading to a collapse in the structure. Figure 5-7 presents a comparison of NR-IPN swelling behaviour at 3\% and 10\% TEGDMA in this context. Hydration is calculated on a dry mass basis, which is measured after surfactant removal. The lines in Figure 5-7 correspond to the estimated points where the water volume added is equivalent to the volume of surfactant and water lost to washing and drying for the $3: 2: 5 \mathrm{NR}$ (solid) and 2:1:1 NR (dashed) formulations, respectively. Swelling at 3\% TEGDMA exceeds these points; however, swelling at $10 \%$ TEGDMA does not meet these thresholds. This may indicate that some of the water in the swollen IPN is present as free water. This could provide an explanation for the irregular domains seen in Figure 5-5, as LSCM imaging does not distinguish between bound and free sodium fluorescein solution. If washing and swelling the NR-IPN essentially reduces and 
restores tension to the structure, then Figure 5-5 may provide evidence of a swollen IPN where the structure has yet to recover from the "collapsed" state.

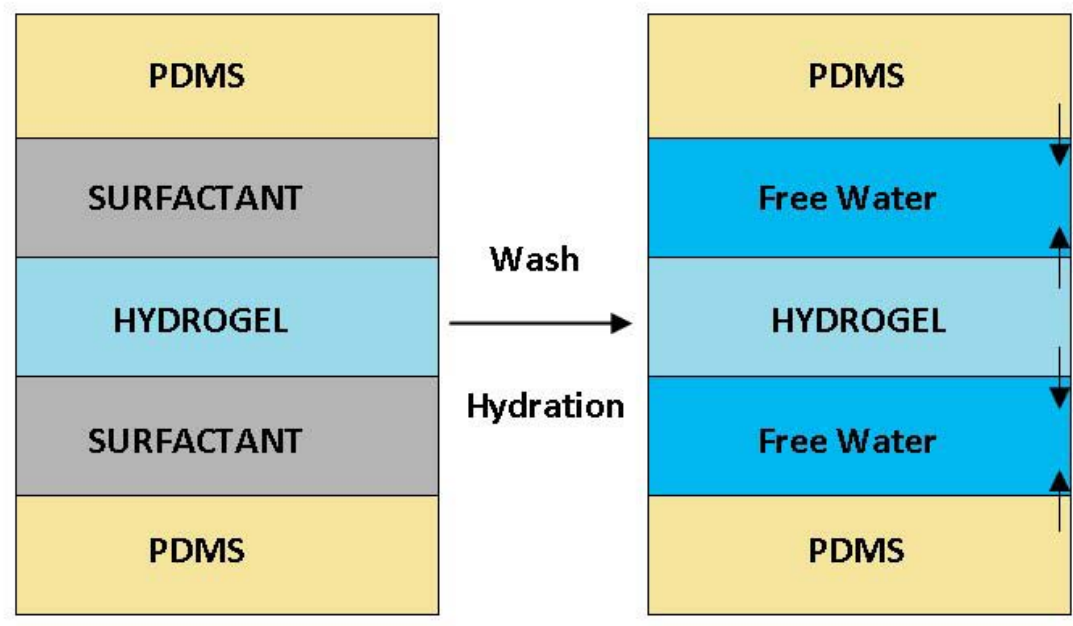

Polymer
Expansion

Figure 5-6: Schematic representation of surfactant removal and replacement with free water in NR-IPN, and polymer expansion/hydration/relaxation.

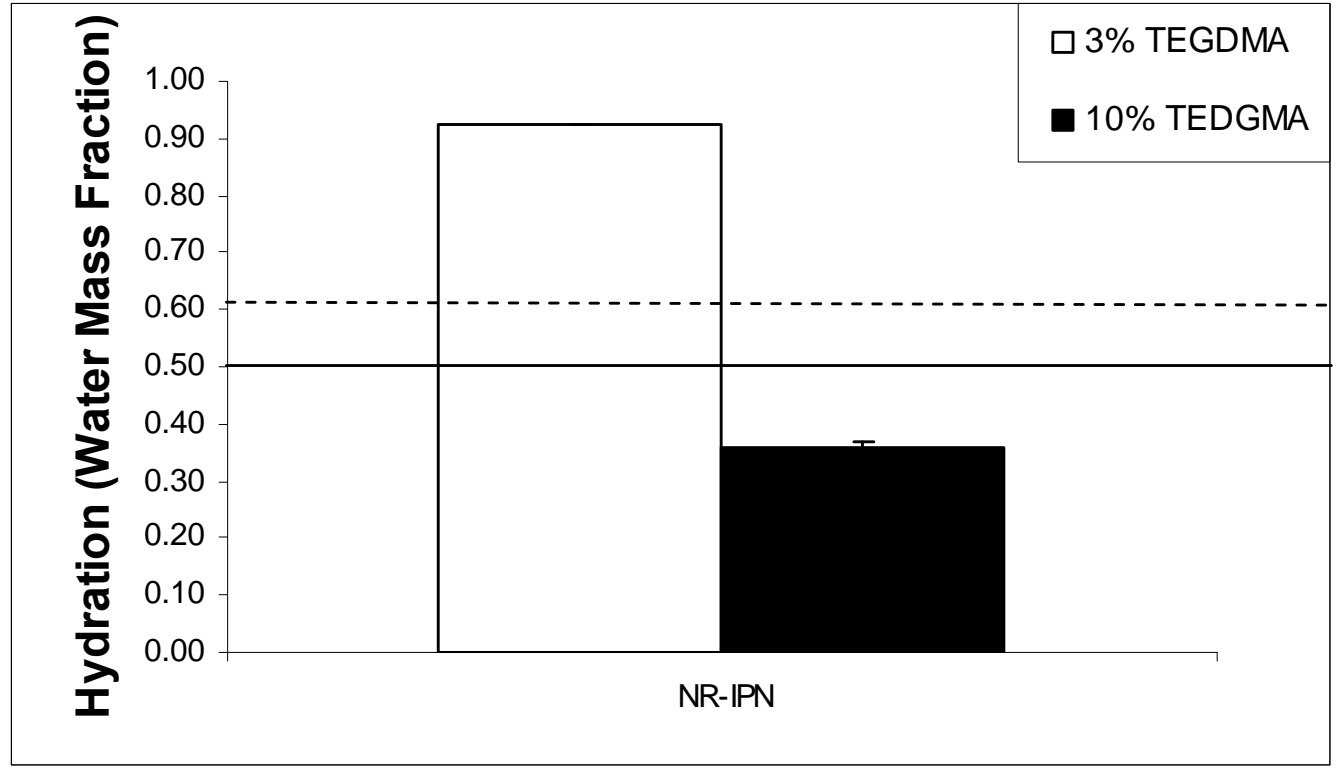

Figure 5-7: NR-IPN swelling comparison at 3\% and 10\% TEDGMA. Approximate swelling space generated by surfactant removal denoted by solid line $(3: 2: 5 \mathrm{NR}$ formulation) and dashed line (2:1:1 NR formulation). 


\subsection{TRANSMISSION ELECTRON MICROSCOPY REVEALS NANOSCALE FRACTURING}

In the previous chapter, cloudiness in dry IPNs was attributed to potential changes in phase behaviour, and thus morphology, as polymerization progresses. Confocal microscopy on swollen samples provided a basis for comparing swollen morphologies. However, it is important to note that the swollen materials contain significant amounts of water, and thus may not provide an accurate representation of dry morphology. Transmission electron microscopy (TEM) imaging of dried NR-IPN samples may provide some insight into the organization of components at the nanoscale, and to the morphology that leads to IPN cloudiness,

\subsubsection{Experimental}

NR-IPNs with 5\% v/v TEGDMA were prepared from microemulsion templates with a surfactant: oil: aqueous ratio of 4:3:3 according to the protocol described in section 4.2.4. Samples were rinsed in distilled water and acetone, and were dried at $110^{\circ} \mathrm{C}$ for 72 hours. Samples were then sectioned using a Leica EM UC6 cryoultramicrotome. $90 \mathrm{~nm}$ thick sections were examined in an FEI Tecnai 20 Transmission Electron Microscope at $100.00 \mathrm{kV}$. 


\subsubsection{Results}

Sample TEM images, as shown in Figure 5-8, indicate several levels of heterogeneous nanostructure, as well as significant fracturing. At the smallest scale, Figure 5-8b reveals interpenetrating channels of $<30 \mathrm{~nm}$, with darkened areas indicating higher silicone content. This is the scale of interpenetration that would be expected from the microemulsion template, as described in chapter 4. A second level of organization is visible in both Figure 5-8A and Figure $5-8 \mathrm{~B}$, with thin, darkened boundaries separating domains of $<100 \mathrm{~nm}$. These boundaries may indicate the presence of denser silicone rubber channels, residual surfactant, and the dispersed platinum catalyst. At the largest scale, gaps of $>80 \mathrm{~nm}$ separate pockets of polymer. This is most evident in Figure 5-8B, where a $200 \mathrm{~nm}$ IPN flake has separated from the main body.

The scale of internal fracturing could provide an explanation for the apparent macroscopic cloudiness of dry NR-IPNs. Some fracturing can be attributed to sectioning and handling of samples. However, it is likely that the extraction of surfactant, untangled oligomer and excess monomer through washing contributes to the large gaps, essentially leading to the formation of grain boundaries. In effect, the sea-island morphology with loosely connected pockets of IPN defines the characteristic domain size of the material as is relevant to optical transparency, despite the scale of silicone-hydrogel interpenetration within these pockets. In addition, the gaps provide supporting evidence for the discussion in the previous section. 


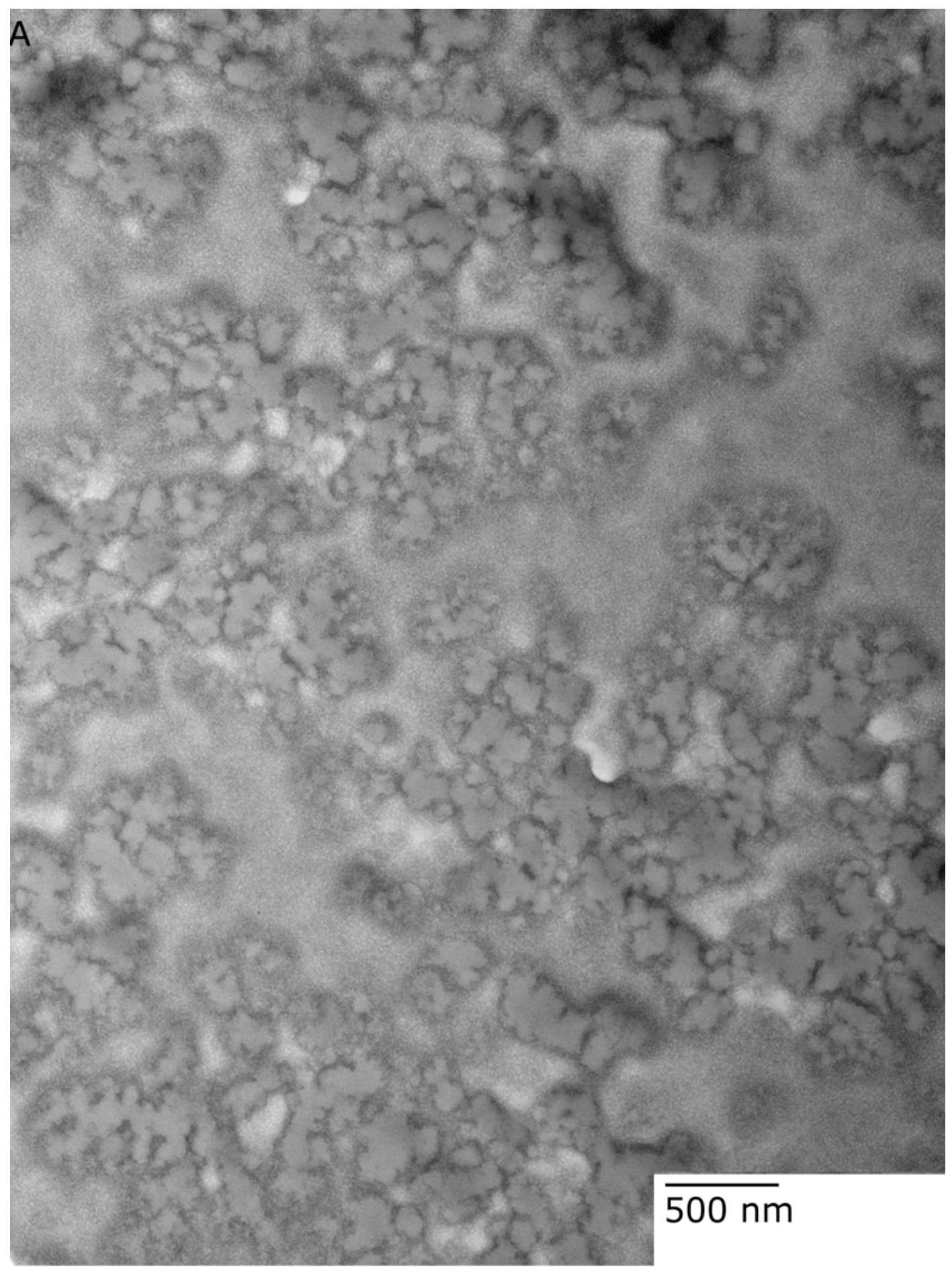




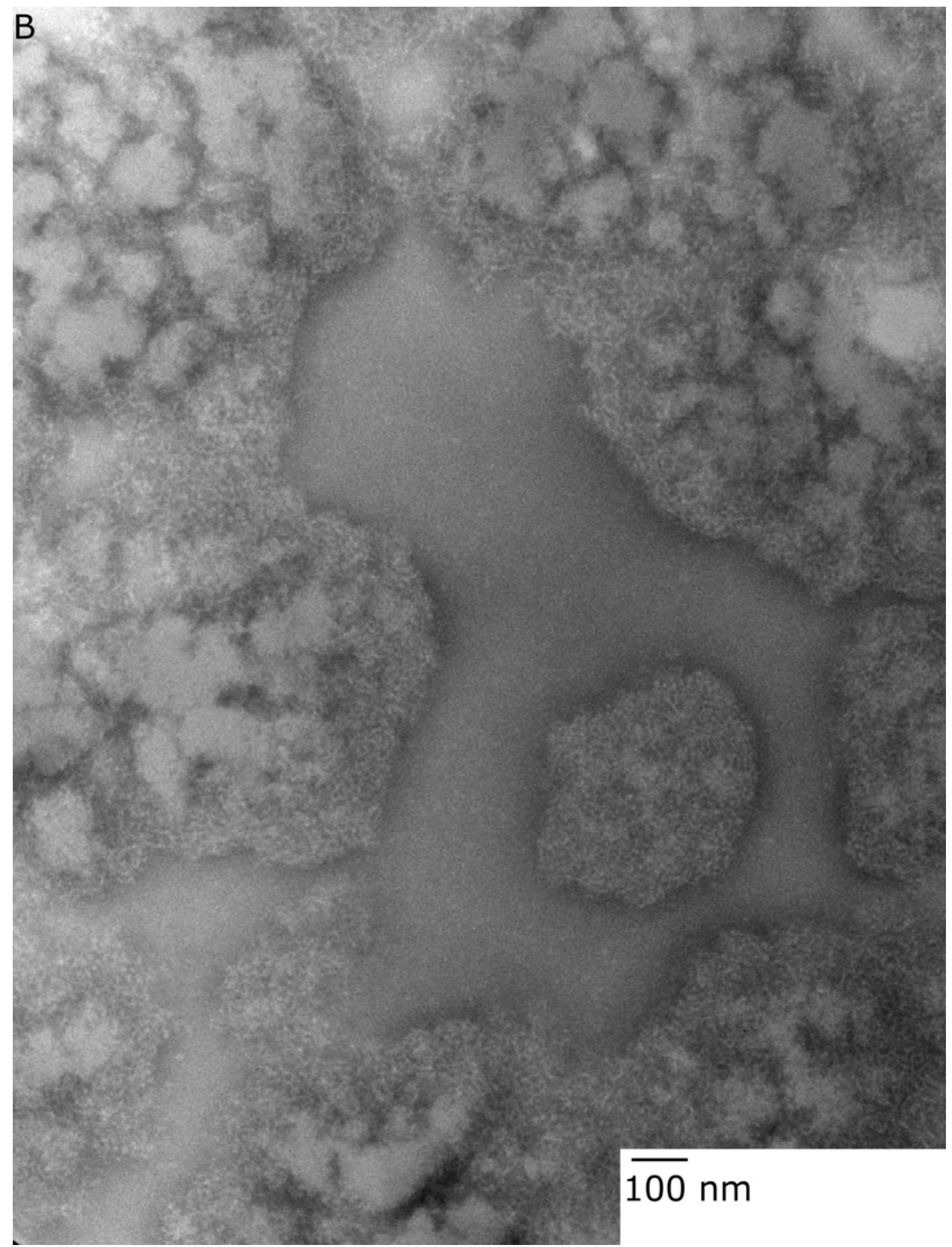

Figure 5-8: TEM images of dry NR-IPN, 5\% v/v TEGDMA. Darkened areas are associated with higher silicone content. ${ }^{9}$ A) IPN nanostructure is heterogeneous, but contains gaps. B) Fracturing and interpenetration at higher magnification. 


\subsection{CONCLUDING REMARKS}

LSCM images of polymers generated from four different compositions in the pseudo-ternary phase diagram were evaluated in context with their microemulsion templates. The polymers showed a variation in domain size associated with monomer consumption and migration as polymerization progresses, indicating a deviation from the initial microemulsion template consistent with results reported in literature ${ }^{1-6}$. This effect is particularly evident in the PDMS domains. However, the swollen morphologies were also found to reflect the initial state of the microemulsion template at $25^{\circ} \mathrm{C}$, providing further evidence that the polymerization-induced increase in viscosity aids in restricting changes in the microemulsion phase behaviour and morphology. Formulations that formed bicontinuous microemulsions yielded interpenetrating morphologies, while an oil-in-water micellar formulation yielded large PDMS domains within a hydrogel matrix.

TEM imaging of dry NR-IPN sections revealed gaps in the structure, attributed to the washing process. The images indicate that the extraction of surfactant, untangled oligomer and excess monomer does not affect the IPN uniformly. 


\subsection{REFERENCES}

1. Peinado, C.; Bosch, P.; Martin, V.; Corrales, T. J. Polym. Sci. Part A 2006, 18, 5291-5303.

2. Stubenrauch, C.; Tessendorf, R.; Strey, R.; Lynch, I.; Dawson, K. A. Langmuir 2007, 14, $7730-7737$.

3. Stubenrauch, C.; Tessendorf, R.; Salvati, A.; Topgaard, D.; Sottmann, T.; Strey, R.; Lynch, I. Langmuir 2008, 16, 8473-8482.

4. Magno, M.; Tessendorf, R.; Medronho, B.; Miguel, M. G.; Stubenrauch, C. Soft Matter 2009, 23, 4763-4772.

5. Gao, F.; Ho, C.; Co, C. C. Macromolecules 2006, 26, 9467-9472.

6. Summers, M.; Eastoe, J.; Davis, S.; Du, Z.; Richardson, R. M.; Heenan, R. K.; Steytler, D.; Grillo, I. Langmuir 2001, 17, 5388-5397.

7. Turner, J. S.; Cheng, Y. -L. Macromolecules 2003, 6, 1962-1966.

8. Liu, L.; Sheardown, H. Biomaterials 2005, 3, 233-244.

9. Wang, J.; Li, X. J Appl Polym Sci 2010, 5, 2749-2757. 


\section{SUMMARY OF FINDINGS}

Through this thesis project, I have detailed the development of a simple process for simultaneous polymerization of PDMS-P(MAA-HEMA) IPNs templated on bicontinuous microemulsions and stabilized by silicone alkyl polyether and silicone acrylate surfactants. The project expands on the classical definition of IPNs to a scale of entanglement at the level of groups of polymer chains, as opposed to molecular or chain-level entanglement. This is a consequence of using the distinct aqueous and oil domains of a microemulsion as the template in place of the intimate molecular mixing associated with conventional IPN synthesis. The implication is that while domain sizes for conventional IPNs increase as spinodal decomposition proceeds, microemulsion templated IPN domain sizes ideally remain constant through polymerization. The primary hypothesis guiding this work was as follows:

\section{If the interpenetrating morphology of a bicontinuous microemulsion containing reactive monomers is controlled and stabilized, then polymerization of both phases will yield an interpenetrating polymer network.}

A series of objectives were developed and satisfied in order to test this hypothesis, leading to the formulation and polymerization of a bicontinuous microemulsion into a silicone - hydrogel IPN. In addressing the first objective, a consistent, quantifiable basis for understanding and formulating silicone microemulsions, the HLD framework, was applied to the characterization of silicone oils and silicone surfactants. The HLD equations were used to characterize silicone oils by an EACN and silicone alkyl polyether surfactants by a characteristic curvature. The role of the MAA and HEMA as both surface active agents and solutes was evaluated qualitatively 
according to HLD shifts, and quantitatively through the co-surfactant function for nonionic surfactant system, $\varphi(\mathrm{A})$. The effect of counter-ion selection on microemulsion phase behaviour was assessed, with the result that while a smaller cation produces a larger shift in HLD, the overall effect on stability is not significant. Finally, the formation of a reactive, bicontinuous microemulsion containing MAA and HEMA, confirmed by conductivity measurement, has also been demonstrated, and its phase behaviour has been investigated with respect to composition, temperature and $\mathrm{pH}$. Characterization indicates a link between increasing monomer content and a higher required surfactant hydrophobicity. The approach to silicone microemulsion formulation and characterization presented here is intended as general method for characterizing silicone oils and derivatives in the future.

In addressing the second major objective, the reactive silicone microemulsions were successfully polymerized through simultaneously radical polymerization. Using the techniques and knowledge generated from characterization work described above, a low viscosity, reactive bicontinuous microemulsion was formulated that yields a hydrogel-PDMS IPN in one polymerization step. The swelling behavior and permeability of the resulting polymer materials indicate the formation of a hydrogel phase. LSCM on IPNs swollen in sodium fluorescein solution confirms connectivity in the aqueous phase, and also confirms that both phases are well distributed with swollen domain sizes of $<1 \mu \mathrm{m}$. The introduction of polymerizable surfactants can aid in stabilizing and preserving the structure of the bicontinuous microemulsion template, reducing swollen domain size to $<200 \mathrm{~nm}$ in diameter. LSCM images of polymers generated from four different locations in the ternary phase diagram indicate were found to reflect the initial state of the microemulsion template at $25{ }^{\circ} \mathrm{C}$. Formulations that formed bicontinuous 
microemulsions yielded interpenetrating morphologies, while an oil-in-water micellar formulation yielded large PDMS domains within a hydrogel matrix.

As described in Chapter 1, the primary motivation for this work is the development of biomaterials suitable for use in physiological fluid-contacting applications. The findings presented in this thesis open the door to formulations combining a variety of hydrophilic materials with PDMS. More importantly, the dependence of microemulsion structure on phase behavior allows for range of possible templates. It is worthwhile to note that the surfactants used in this work were not designed for use in microemulsions, and it is our hope that continued progress in the study of reactive microemulsions will encourage the development and characterization of more appropriate surfactants.

\subsection{FUTURE WORK}

The findings of this work have led to several topics of interest that warrant further investigation, and these are listed below.

- The two fundamental challenges in developing polymer blends, mixtures, or IPNs can be summarized as: A) Overcoming the enthalpy driven miscibility gap and B) Overcoming thermodynamically driven temperature or polymerization induced phase separation. The low interfacial tensions offered by microemulsions provide an opportunity to address A, while it has been shown that permanent entanglements, vitrification or even 
copolymerization can address B. However, the thermodynamics of microemulsions and the thermodynamics of polymer solutions, while separately well studied, are not yet linked. An important contribution to both fields would involve investigating and modeling microemulsion thermodynamics for different formulations and structures as they relate to a reduction in miscibility gap for polymer solutions.

- As it has been shown that the HLD can be extended to characterization of silicone microemulsions components, it follows that the next step should involve continued attempts to characterize oils and surfactants. In particular, the characterization of higher molecular weight oils is important to the development of biomaterials. A larger library of components allows for a wider range of formulations. However, solubility limitations will necessitate the development of new reference systems. In addition to characterization through empirical fitting, it is important to develop a robust, theoretical conception of how each HLD parameter relates to the underlying thermodynamics of microemulsion behaviour.

- The addition of reactive monomers to the aqueous phase resulted in a shift in HLD, as the aqueous phase essentially becomes less "water-like." While the co-surfactant function, $\varphi(A)$ was estimated from a linear approximation, further research into an accurate method for evaluating $\varphi$ (A) for a variety of polymers will be necessary. This would require both an empirical approach to fit parameters, and a more fundamental approach to determine the molecular properties of the monomer that define the function. In particular, it is worth determining experimentally the location and interactions of each monomer at the interface in order to more accurately describe their role as linkers. The investigation of the co-surfactant behaviour raise the interesting question of whether the 
co-surfactant function $\varphi(\mathrm{A})$ should instead be considered a characteristic value of the polar phase, analogous to the EACN.

- The surfactants used in this project were not designed for applications involving microemulsions. However, by assigning them characteristic values according to their hydrophobicity, they were successfully incorporated into bicontinuous formulations. An important contribution to the field of silicone surfactants would be the design and synthesis of versatile silicone surfactants that more accurately reflect the range of requirements for a silicone microemulsion. The HLD, and other theoretical frameworks, provide a basis for comparison with hydrocarbon surfactants. However, silicone surfactant design has yet to be linked explicitly to requirements of common hydrocarbon surfactants, such as AOT.

- The work with polymerizable surfactants presented in this thesis raises an important question: Which components of the microemulsion are necessary? It was found that reactive formulations could be generated using only silicone surfactant, oil, and reactive monomer. Even simpler systems could be developed using only surfactant and monomer. The range of structures that can be polymerized from these systems strongly warrants investigation, as this represents an even simpler pathway to silicone-hydrogel IPN synthesis than the methodology presented here. In addition, the incorporation of polymerizable surfactants into the hydrogel introduces the possibility of "reversing" conventional sequential silicone-hydrogel synthesis, with the hydrogel as the host polymer.

- The eventual goal of developing suitable materials for biomedical and drug delivery devices will require further development of the framework described here. In particular, 
the polymerization process and selection of components should be refined to generate transparent materials with greater consistency. This is particularly important in the development of contact lens materials. Moreover, the relationship between formulation components and transport properties needs to be further investigated to validate materials intended as coatings, membranes, microfluidic substrates, or drug delivery devices.

- The characterization and formulation framework used in this work is not intended to be limited to MAA, HEMA and low viscosity silicone oil. These components were selected to demonstrate the level of complexity that this framework can address. Future work should investigate the use of alternate hydrophilic monomers and crosslinkers in template formulation that are specific to the desired application. 


\section{APPENDIX A: Surfactant and Oil Characteristic Values}

Appendix A-1: Characteristic Curvatures for Selected Surfactants

\section{Surfactant}

Ce

Sodium mono- and di-methyl naphthalene sulfonate (SMDNS)

Sodium naphthenate (Sigma)

Sodium naphthenate (Kodak)

Sodium dodecyl sulfate (SDS)

Sodium octanoate

$-3.5$

3.5

$-2.65$

$-2.39$

$-2.36$

$-2.07$

Sodium oleate

$-1.7$

SDHS

$-0.92$

Sodium dodecyl benzene sulfonate

$-0.94$

Naphthenic acid (Sigma)

Oleic acid

$-0.34$

Dodecanol

0.18

Sodium dioctyl sulfosuccinate (AOT) 
Appendix A-2: Selected EACNs

\begin{tabular}{ll|ll}
\hline Oil & EACN & Oil & EACN \\
\hline n-Alkane & \# of carbons & Toluene & 1 \\
Methane-Butane & $\mathrm{f}(\mathrm{P}, \mathrm{T})$ & Napthalene & 1 \\
$\mathrm{CO}_{2}$ & $\mathrm{f}(\mathrm{P}, \mathrm{T})$ & Asphalthenes & 1 \\
$\mathrm{CH}_{2} \mathrm{Cl}_{2}$ & $-13.8^{\mathrm{a}}$ & PCE & 2.9 \\
$\mathrm{CHCl}_{3}$ & $-13.7^{\mathrm{a}}$ & Cyclohexane & 3 \\
$\mathrm{DCB}$ & $-4.9^{\mathrm{a}}$ & Ethyl Oleate & 6 \\
$\mathrm{Trichloroethylene}$ & $-3.8^{\mathrm{a}}$ & Limonene & $1.6-6.8$ \\
$\mathrm{CCl}_{4}$ & $-0.06^{\mathrm{a}}$ & Maltenes & 6 \\
Benzene $_{\text {Paraffin }(\mathrm{C} 17-30)}$ & 17.7 & Corn Oil & 16 \\
\hline
\end{tabular}

${ }^{a}$ As the EACN scale is calibrated to $n$-alkanes, the EACN value only indicates a relative hydrophobicity. Thus a negative EACN implies that the oil is more hydrophilic than benzene. 
Appendix A-3: Terpene EACNs

\begin{tabular}{ll|ll}
\hline Oil & EACN & Oil & EACN \\
\hline Myrcane & 9.3 & $\beta$-Pinene & 1.9 \\
2,6,10-Trimethyl undecane-2,6diene & 8.6 & $\Delta$-3-Carene & 1.9 \\
Longifolene & 6.1 & Limonene & 1.6 \\
p-Menthane & 5.7 & $\gamma$-Terpinene & 1.4 \\
Caryophyllene & 5.3 & $\alpha$-Terpinene & 0.9 \\
Pinane & 3.4 & Terpinolene & 0.3 \\
p-Menth-2-ene & 3.2 & p-Cymene & $-0.8^{\text {a }}$ \\
a-Pinene & 3.0 & & \\
\hline
\end{tabular}

${ }^{a}$ As the EACN scale is calibrated to $n$-alkanes, the EACN value only indicates a relative hydrophobicity. Thus a negative EACN implies that the oil is more hydrophilic than benzene.

References:

V. Nardello, N. Chailloux, J. Poprawski, J.-L. Salager, J.-M. Aubry, Polym Int. 52 (2003) 602.

F. Bouton, M. Durand, V. Nardello-Rataj, M. Serry, J. Aubry, Colloids Surf Physicochem Eng Aspects. 338 (2009) 142.

Kiran, S. K.; Acosta, E. J.; Moran, K. J. Colloid Interface Sci. 2009, 1, 304-313.

Acosta, E. J.; Yuan, J. S.; Bhakta, A. S. Journal of Surfactants and Detergents 2008, 2, 145158.

Acosta, E. J.; Bhakta, A. S. Journal of Surfactants and Detergents 2009, 1, 7-19. 


\section{APPENDIX B: Sample Fish Diagram}

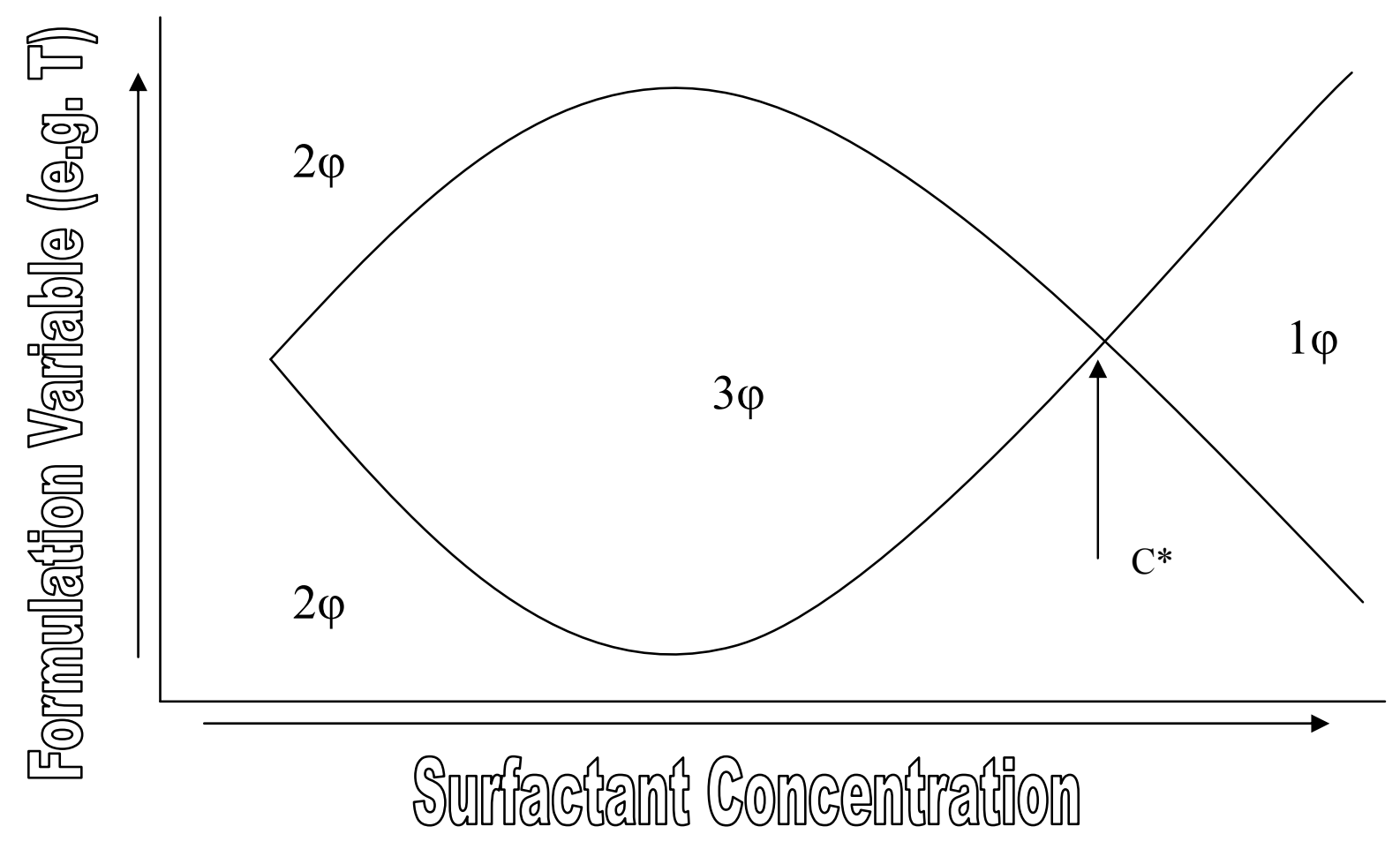

